\title{
ION BEAM MODIFICATION OF METALS: COMPOSITIONAL AND MICROSTRUCTURAL CHANGES
}

\author{
GARY S. WAS \\ Departments of Nuclear Engineering and Materials Science and Engineering, \\ University of Michigan, Ann Arbor, MI 48109
}

\begin{abstract}
Ion implantation has become a highly developed tool for modifying the structure and properties of metals and alloys. In addition to direct implantation, a variety of other ion beam techniques such as ion beam mixing, ion beam assisted deposition and plasma source ion implantation have been used increasingly in recent years. The modifications constitute compositional and microstructural changes in the surface of the metal. This leads to alterations in physical properties (transport, optical, corrosion, oxidation), as well as mechanical properties (strength, hardness, wear resistance, fatigue resistance). The compositional changes brought about by ion bombardment are classified into recoil implantation, cascade mixing, radiation-enhanced diffusion, radiation-induced segregation, Gibbsian adsorption and sputtering which combine to produce an often complicated compositional variation within the implanted layer and often, well beyond. Microstructurally, the phases present are often altered from what is expected from equilibrium thermodynamics giving rise to orderdisorder transformations, metastable, (crystalline, amorphous or quasicrystalline) phase formation and growth, as well as densification, grain growth, formation of a preferred texture and the formation of a high density dislocation network. All these effects need to be understood before one can determine the effect of ion bombardment on the physical and mechanical properties of metals. This paper reviews the literature in terms of the compositional and microstructural changes induced by ion bombardment, whether by direct implantation, ion beam mixing or other forms of ion irradiation. The topics are introduced as well as reviewed, making this a more pedogogical approach as opposed to one which treats only recent developments. The aim is to provide the tools needed to understand the consequent changes in physical and mechanical properties.
\end{abstract}




\section{Contents}

1. Introduction

2. Ion Beam Modification Techniques

3. Compositional Changes

A. Recoil implantation $\quad 220$

B. Cascade mixing 224

C. Chemical effects 229

D. Radiation-enhanced diffusion 233

E. Radiation-induced segregation $\quad 239$

F. Gibbsian adsorption $\quad 256$

$\begin{array}{ll}\text { G. Sputtering } & 258\end{array}$

H. Phenomenological model for surface composition changes 262

$\begin{array}{ll}\text { I. Implant redistribution during ion implantation } & 268\end{array}$

$\begin{array}{ll}\text { 4. Microstructural Changes } & 270\end{array}$

$\begin{array}{ll}\text { A. Phase stability } & 271\end{array}$

B. Metastable phases $\quad 286$

C. Additional microstructural effects of ion implantation 314

$\begin{array}{ll}\text { 5. Summary } & 322\end{array}$

$\begin{array}{ll}\text { References } & 324\end{array}$

\section{Abbreviations}

AES Auger electron spectroscopy

bcc body centered cubic

CSRO Chemical short range order

DM Displacement mixing

fcc face centered cubic

GA Gibbsian adsorption

hcp hexagonal close packed

IBAD Ion beam assisted deposition

IBM Ion beam mixing

PS Preferential sputtering

PSII Plasma source ion implantation

RED Radiation enhanced diffusion

RIS Radiation-induced segregation

TEM Transmission electron microscopy

TSRO Topological short range order 
The use of ion beams for altering the properties of materials was pioneered in the 1960s with the introduction of electrically active elements into silicon and other semiconducting materials [1]. It became widely adopted by the microelectronics industry in the 1970s and has developed into a well established and precision industry [2]. The first application of ion implantation into metals was reported in 1969 by Dearnaley [3] at the Harwell Laboratory, who addressed the possibility of improving mechanical and corrosion behavior of steels that are of relevance to the nuclear industry. Rapidly thereafter, the attributes of ion implantation as a surface modification technique became realized and activity in ion implantation of metals mushroomed.

In comparison with alternative surface modification techniques, ion implantation has a number of advantages: 1) the process is inherently low temperature and thermal distortion of components is not a problem, 2) since ion implantation is not a coating process, there is no interface that may become susceptible to decohesion due to mechanical stress or corrosion, 3) dimensional changes are negligible on an engineering tolerance scale, being on the order of a few tens of nanometers, 4) surface polish is improved due to preferential sputter erosion of asperities, 5) the implanted atoms are dispersed on a microscopic (and sometimes on an atomic) level producing the most efficient and beneficial effect of the additive, and 6) significant compressive surface stresses are produced which will partially compensate externally imposed tensile stresses and lengthen component life against creep or fatigue failure by surface initiated cracking.

In addition to these practical attributes, a more general attribute of the implantation process is that it is a non-equilibrium process. As a result the implanted target may form non-equilibrium or metastable phases or microstructures. The study of these microstructures has generated a great deal of interest with regard to the mechanical and physical properties they possess. For example, the superior corrosion properties which accompany formation of an amorphous surface are of tremendous interest because of the ease with which amorphization can occur in specific systems.

Similarly, the mechanical properties of these phases as well as metastable microstructures is of significant interest. Numerous examples exist on the hardening of alloys by ion implantation and ion beam mixing. In many cases, this translates into improved friction and wear properties of well-known engineering materials. The change in microstructure of the alloy surface has also been found to strongly affect the fatigue and creep behavior of the component - 
mechanical responses which are often associated with bulk properties of the sample rather than surface properties.

However, all of the observed property changes in metals are due to either compositional, microstructural or topographical changes in the alloy. We must therefore, seek to understand how ion implantation induces these changes before we can hope to understand their effects on the mechanical or physical properties of alloys. Having accomplished this, we may then look forward to this technique becoming a useful and reliable processing tool for the metals industry.

The purpose of this paper is to present an introduction and a review of the state of ion beam surface modification of metals for property improvements. As just discussed, property improvements can only be understood through a thorough knowledge of the compositional and microstructural changes induced by ion bombardment of metals. Therefore, this topic is divided into two parts. In this, the first part, compositional and microstructural effects of ion bombardment in metals are reviewed. The processes which govern the composition profile of the implanted specie; sputtering, displacement mixing, radiation enhanced diffusion, radiation induced segregation, Gibbsian adsorption and preferential sputtering, and the microstructural development; phase stability, metastable phase formation, amorphization, dislocation dynamics, precipitation, grain growth, etc. are discussed and reviewed.

The second part will review the chemical; corrosion and oxidation, and mechanical; hardness, wear, fatigue, property changes brought about by the compositional and microstructural changes discussed here. The intent is not merely to survey the current literature, but to present a coherent and semiquantitative description and analysis of the physical processes occurring during, or as a result of the ion bombardment process. Hence, a balance is struck between the development of physical models and surveying the current literature, resulting in a more pedagogical presentation.

\section{Ion Beam Modification Techniques}

The modification of metal surfaces by ion beams can be accomplished by a variety of methods, each with its own advantages for particular situations. The principal methods include 1) direct ion implantation, 2) ion beam mixing, 3) ion beam assisted deposition and 4) plasma ion implantation and are shown schematically in Figs. 1-4. Each of these will be briefly described and considered with respect to its advantages and disadvantages. 


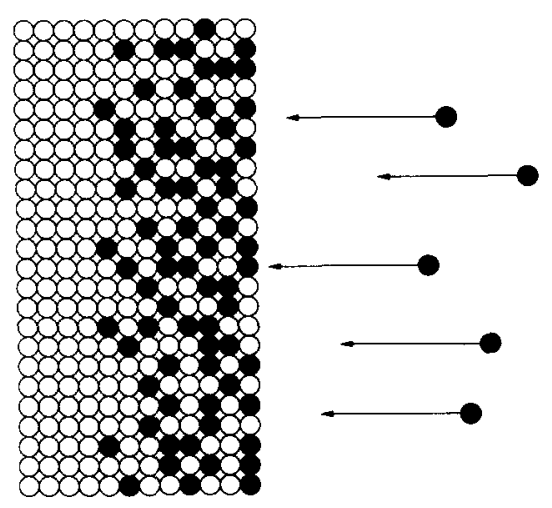

Fig. 1. Schematic representation of direct ion implantation.

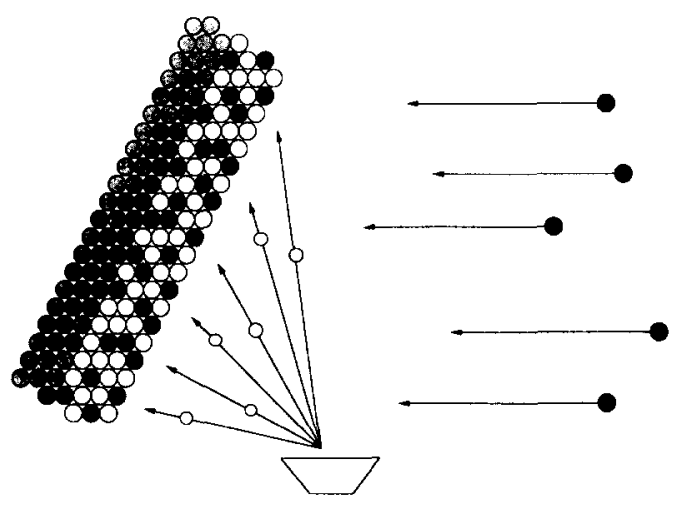

Fig. 3. Schematic representation of ion beam assisted deposition (IBAD).

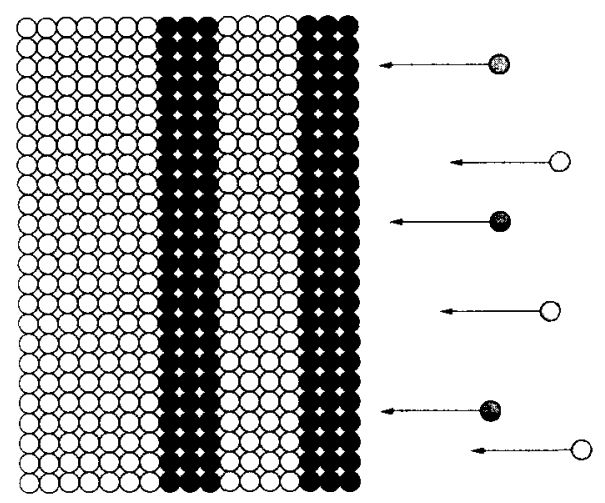

Fig. 2. Schematic representation of the ion beam mixing process (IBM).

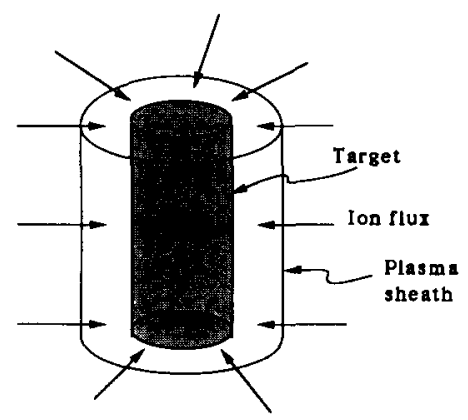

Fig. 4. Schematic representation of the plasma source ion implant ation process (PSII). 
Direct ion implantation is accomplished by bombarding the target with a beam of ions in the energy range from a few hundred $\mathrm{keV}$ to several $\mathrm{MeV}$. The beam is usually monoenergetic, contains a single charge state and is generally (but not always) mass analyzed. Due to the stochastic nature of the elastic collision process, the ions come to rest in a Gaussian distribution with the mean of the Gaussian centered about $R_{p}$, the projected range, with the FWHM $2.35 \Delta R_{p}$ where $\Delta R_{p}$ is the standard deviation from the mean.

Although seemingly a very simple process, this technique has several disadvantages from the standpoint of modifying the surface composition. First, the depth of the implanted distribution varies as $E^{1 / 2}$ and hence, energies in the several hundred $\mathrm{keV}$ range, achievable in the most common implanters, will only result in projected ranges of the order of $100 \mathrm{~nm}$ for many ions. MeV energies are needed to penetrate the micron range with heavy ions. Second, sputtering will limit the concentration of the implanted specie to a value that is the reciprocal of the sputtering yield. Since sputtering yields of metals at these energies range from 2-5, the maximum concentration of implanted specie is $50 \%$ to $20 \%$ respectively. The shape and location of the distribution can also be a problem. In corrosion, where the composition of the top monolayer is most important, the bulk of the modification occurs at considerably greater depths, leaving the surface lean in the implanted specie. When the implantation induces a phase transformation as well, the effectiveness or efficiency of direct implantation is lesser still. Finally, it is often desirable to implant metal ions into pure metals or alloys to achieve particular surface compositions. As a practical matter, most commercial implanters can produce large currents of inert gases, but more elaborate measures are needed in order to produce metal ions at currents which are practical. Ion beam mixing provides an alternative to the shortcomings of direct ion implantation.

Ion beam mixing (IBM) refers to the homogenization of bilayers or multilayers of elements deposited onto the surface of a target prior to bombardment. The idea behind IBM is to create a surface alloy by homogenizing alternate layers of the alloy constituents deposited in a thickness ratio so as to result in the desired final composition following mixing. This greatly relieves several of the shortcomings of direct implantation. First, the requirement of producing a metal ion beam is eliminated since noble gases can be used for the mixing. They are not expected to contribute a chemical effect in the solid and yet can be made into high current beams in most commercial implanters. Second, there is no restriction on the composition range since the final composition is controlled by the ratio of layer thicknesses. This also removes two other shortfalls of direct implantation; that of uniformity and surface 
deficiency of the implanted specie. Ion beam mixing results in a very uniform composition throughout the depth of the penetration. This includes the very near surface region which is a problem with direct implantation. Finally, if the elemental layers are made thin enough, the dose needed to achieve complete mixing can be orders of magnitude lower than that needed to produce concentrated alloys by direct implantation.

One further point is in regard to phase formation during or following ion beam mixing. If the process is carried out at low temperature, the result is often a metastable alloy in the form of either a supersaturated solid solution or an amorphous structure. The microstructure can then be controlled by subsequent annealing treatments. However, despite its many advantages, ion beam mixing still suffers from the common disadvantage of limited depth of penetration. The thickness of the surface is still governed by the projected range of the ion which is in the $100 \mathrm{~nm}$ range for few hundred $\mathrm{keV}$ heavy ions. A solution to this problem lies in the technique of ion beam assisted deposition.

Ion beam assisted/enhanced deposition (IBA/ED) refers to the growth of a film with the assistance of an ion beam. In this technique, which is reviewed extensively in Smidt [4], a film is grown onto a substrate by physical vapor deposition using either an electron beam gun or an effusion cell, concurrently with the bombardment by an ion beam. The advantages of this method are numerous. First, there is virtually no limit to the thickness of film which can be modified since modification occurs during growth. Second, ion bombardment concurrent with vapor deposition provides for an atomically mixed interface, resulting in greater adherence. The composition gradient in the interface can be controlled by the deposition rate and the ion flux. Third, the enhanced mobility of the surface during growth allows for the control of grain size and morphology, texture, density, composition, and residual stress state. These properties are also controlled principally by controlling the atom deposition rate in conjunction with the ion flux (ion to atom arrival rate ratio), ion energy, fluence and species. Hence, pure metals, solid solution alloys, intermetallic compounds and a host of metal-base compounds can be grown by this technique.

A final technique which has recently been developed is plasma surface ion implantation (PSII) [5]. In this technique, targets to be implanted are placed directly in the plasma source and are then pulse biased to a high negative potential $(-40 \mathrm{keV}$ to $-100 \mathrm{keV})$. A plasma sheath forms around the target and ions are accelerated normal to the target surface, across the plasma sheath [5]. PSII has several potential advantages relative to conventional line-of-sight implantation including 1) elimination of the need for target manipulation and beam rastering, 2) elimination of target masking (retained dose problem), 3) the 
ion source hardware and controls are near ground potential, and 4) PSII is expected to be readily scaled to large and/or heavy targets.

However, all of these techniques involve energetic ion-solid interaction and the physical processes that accompany this interaction. The following sections address physical processes which affect compositional changes; recoil implantation, cascade mixing, radiation enhanced diffusion, radiation-induced segregation, Gibbsian adsorption and sputtering; and microstructural and phase changes; phase stability, metastable phase formation and amorphization, radiation induced grain growth, precipitation and dislocation dynamics. These compositional and microstructural changes form the basis for the observed changes in physical and mechanical properties of the metal or alloy of interest.

\section{Compositional Changes}

Immediately upon entering the solid, the ion begins to lose energy by electronic excitation of the atomic electrons of the host atoms and by elastic collisions with the shielded atomic nuclei. In metals, the electronic energy loss is a major contributor to the slowing down of the ion, but it is the elastic collisions which lead to atomic displacements. At high energy, the elastic collision crosssection is low compared to the electronic energy transfer cross section and the majority of slowing down is by electronic excitation. Those elastic collisions which occur produce primary knock-on atoms in the $\mathrm{keV}$ energy range. As the energy of the injected ion as well as that of the high energy knock-ons is dissipated through both mechanisms, the elastic collision cross section becomes very large and the mean free path between interactions is reduced to a few Angstroms. The picture of energy transfer by elastic collisions changes from one of sparse (few and widely separated), high energy knock-on production to the production of a high density of low energy knock-ons. The result is a collision cascade in which the kinetic temperature may reach $10^{4}-10^{6} \mathrm{C}$ and in which the atoms interact in a collective fashion producing significant lattice disorder. These dense cascades are characterized by either their temperature (thermal spikes) or atom relocation (displacement spikes).

The temporal development of the cascade can be divided into three phases:

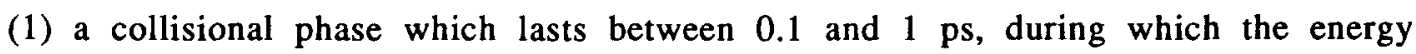
transferred to the primary knock-on atom is dissipated among successive recoils, (2) a relaxational phase of about 0.5 ps during which Frenkel pairs spontaneously recombine due to their close proximity, and (3) a cooling phase lasting only a few ps in which the highly disordered cascade region cools to 
reach equilibrium with the surroundings. The mixing which occurs in the first two phases is termed ballistic, while the third phase involves collective behavior giving rise to the term "thermal spike" to describe the distribution of of energy among the atoms. According to the modified Kinchen-Pease model [6], the number of displacements per target atom per second in a cascade is given by the relation

$$
\mathrm{P}(\mathrm{x})=0.8 /\left(2 \mathrm{NE}_{\mathrm{d}}\right)(\mathrm{dE} / \mathrm{dx})_{\mathrm{n}} \phi,
$$

where $\phi$ is the ion flux, $N$ is the atomic density of the solid, $E_{d}$ is the effective threshold displacement energy and $(\mathrm{dE} / \mathrm{dx})_{\mathrm{n}}$ is the ion energy deposited per unit depth into atomic processes. Within a single cascade, the mean displacement distance is always insignificantly small. For example, if each Frenkel pair is displaced an average of $\sim 10 \AA\left(\mathrm{R}_{\text {recoil }}\right)$, because the ratio of the number of displaced atoms $N_{d}$, to the total number of atoms within the central core of the cascade $N_{v}$, is much less than unity, the mean atomic displacement due to ballistic mixing within the cascade $\left(R_{\text {recoil }} \times N_{d} / N_{v}\right)$ is negligible. Only with the aid of radiation enhanced diffusion (due to the high defect concentration) after the cascade has ended, could significant mixing occur. At doses $>10^{16} \mathrm{ions} / \mathrm{cm}^{2}$, the implanted region receives $\geq 10^{3}$ successive overlapping cascades and the cumulative effect of ballistic mixing is no longer negligible.

The primary disordering mechanism is collisional or ballistic mixing which can be qualitatively classified into recoil implantation and cascade (isotropic) mixing. Recoil implantation refers to the direct displacement of a target atom by a bombarding ion. Indirect processes involving other target atoms are collectively called cascade mixing. Referring to experiments involving the implantation into a bilayer or a thin marker layer embedded in a monatomic solid, recoil implantation produces a shift and a broadening of a given initial profile while cascade mixing produces primarily a broadening. But in addition to collisional mixing, thermal processes may become important, leading to radiation enhanced diffusion in the target and a greater degree of atomic mobility and relocation than at low temperatures. In alloys at higher temperatures, when the flux of defects to sinks becomes coupled to the counter flow of host atoms, radiation-induced segregation can occur, resulting in the accumulation or depletion of an alloy component at defect sinks. Finally, the sputtering of atoms from the bombarded surface results in compositional changes in the near surface region of an alloy and can only be explained using our understanding of the processes just mentioned. The following subsections provide a development as well as a review of the physical processes of recoil implantation, cascade mixing, 
radiation-enhanced diffusion, radiation-induced segregation, Gibbsian adsorption and sputtering.

\section{A. Recoil implantation}

In alloys, the relocation cross section and the range of the recoiling atoms depend on the charge and mass of the nucleus in such a way that generally the lighter component atoms will be transported relative to the heavier components in the beam direction. This can be described as a flux of atoms of some of the alloy components toward deeper regions in the target, compensated by an opposite flux of the remainder of components to maintain atomic density at the proper value, i.e., the net flux of atoms is approximately zero across any plane parallel to the surface inside the target. The expression "recoil implantation" is used here for this net transport parallel to the beam direction of some types of atoms relative to other types. The mechanics of recoil implantation have been developed by Sigmund [7-9]. He showed that an impurity atom knocked off its lattice site by an incoming projectile ion has a relocation cross section that can be estimated from LSS theory $[10,11]$ as

$$
\begin{gathered}
d \sigma_{i j}(E, T)=C_{i j} E^{-m} T^{-1-m_{d} T,} \\
0 \leq T \leq \gamma_{i j} E, \quad i j=0,1,2, \\
\text { and } \gamma_{i j}=4 M_{i} M_{j} /\left(M_{i}+M_{j}\right)^{2},
\end{gathered}
$$

where $C_{i j}$ is a constant, $m$ is a parameter describing the collision $(0<m<1)$ and is usually taken to be a value of $1 / 2$ for interactions in the $\mathrm{keV}$ range and $1 / 3$ in the $\mathrm{eV}$ range, $\mathrm{E}$ is the incoming ion energy and $\mathrm{T}$ is the energy transfer. The impurity will, on average, be found at a depth

$$
\left\langle z+x>=x+\delta \Phi \int z d \sigma(x, z)\right.
$$

after bombardment by a differential fluence $\delta \Phi$, where

$$
z=R_{p}(T) \cos \theta
$$

and $R_{p}$ is the projected range and $\cos \theta=\left(T / \gamma_{01} E\right)^{1 / 2}$. The mean recoil implantation $\langle\mathrm{z}\rangle$ is

$$
<\mathrm{Z}>=(\alpha+1 / 2-m)^{-1} \delta \Phi B(x) z_{m}(x),
$$


and the relocation profile of the impurity distribution is given by:

$$
\mathrm{P}(\Delta \mathrm{x}) \sim \Phi \mathrm{d} \sigma(\mathrm{x}, \Delta \mathrm{x}) / \mathrm{d}(\Delta \mathrm{x}), \text { for large } \Delta \mathrm{x} .
$$

The quantity $B(x)$ is of the order of $\pi r_{0}^{2}$, with $r_{0}$ being the distance of closest approach in an ion-impurity collision at depth $x$, and $\alpha$ is a constant between 0 and 2. As a rule of thumb, $z_{m}$ is less (greater) than the residual ion range $R(x)$ at $x$ if the impurity is heavier (lighter) than the matrix. The total effect $P_{i}$, of this knock-on implantation ( $\int$ number $x$ range) is given by

$$
P_{i}=N c_{i} \int_{0}^{R} d x \int_{0}^{\gamma_{0 i} E(x)} d \sigma_{0 i}[E(x), T] R_{i}\left[(T) / \gamma_{0 i} E(x)\right]^{1 / 2},
$$

where $N$ is the number density of atoms, $c_{i}$ is the concentration of alloy component $i$ and $E(x)$ is the ion energy at depth $x$. The range $R_{i}(T)$ is determined by the partial stopping cross sections $S_{i j}(E)=\int T^{\prime} d \sigma_{i j}\left(E, T^{\prime}\right)$. After integration [7] of eqn. (3.7) one finds

where

$$
\mathrm{Q}_{\mathrm{ij}}=\mathrm{P}_{\mathrm{i}} / \mathrm{R}=\gamma_{0 \mathrm{i}}{ }^{2 \mathrm{~m}-1}(1-\mathrm{m}) / \mathrm{m}(1+2 \mathrm{~m}) \quad\left(\mathrm{c}_{\mathrm{i}} /\left(\left(\mathrm{A}_{\mathrm{i} 1}+\mathrm{A}_{\mathrm{i} 2}\right)\right),\right.
$$

$$
A_{i j}=c_{j}\left(\gamma_{i j} / \gamma_{0 i}\right)^{1-m} \quad\left(a_{i j} / a_{0 i}\right)^{2(1-m)} \quad\left(M_{i}^{2} / M_{0} M_{j}\right)^{m} \quad\left(Z_{j} / Z_{0}\right)^{2 m} .
$$

The quantity $Q_{i}$ is the equivalent number of $i$ atoms recoil implanted over the depth $R$ (i.e. the ion range) in the direction of the ion beam per incident ion. The dominating term in $\mathrm{Q}_{\mathrm{i}}$ with regard to its dependence on the target composition is $M_{\mathrm{i}}{ }^{2 \mathrm{~m}}$. The dependence is such that recoil implantation of the lighter species dominates that of the heavier one. Although the number of $i$ recoils at a given energy ( $\mathrm{T}, \mathrm{dT}$ ) is dominated by the heavy component (as $\mathrm{M}_{\mathrm{i}}^{-}{ }^{-\mathrm{m}} \mathrm{Z}_{\mathrm{i}}{ }^{2 \mathrm{~m}}$ ), the range of $i$ recoils at a given $T$ is a $M_{i}^{-m} Z_{i}{ }^{-2 m}$ and so the combined effect is greatest for the lighter species. Equation (3.5) also indicates that the mean relocation due to recoil implantation should have a linear dependence on fluence.

Sigmund applied this formalism to the case of argon bombarded PtSi [7] and determined that $\mathrm{Q}_{\mathrm{Pt}}=0.034$ and $\mathrm{Q}_{\mathrm{Si}}=0.37$ for $\mathrm{m}=1 / 2$, and $\mathrm{Q}_{\mathrm{Pt}}=0.19$ and $\mathrm{Q}_{\mathrm{Si}}=$ 0.94 for $m=1 / 3$. Both cases predict a net transport of $\mathrm{Si}$ that is considerably greater than that for Pt, Fig. 5. Results of Liau et al. [12] provided initial support for this model. Paine [13] conducted experiments on a sample of the same 
geometry as described by Sigmund [7] using $300 \mathrm{keV} \mathrm{Xe}^{+}$at $90 \mathrm{~K}$ to fluences up to $1.27 \times 10^{16} \mathrm{i} / \mathrm{cm}^{2}$, Fig. 6 .

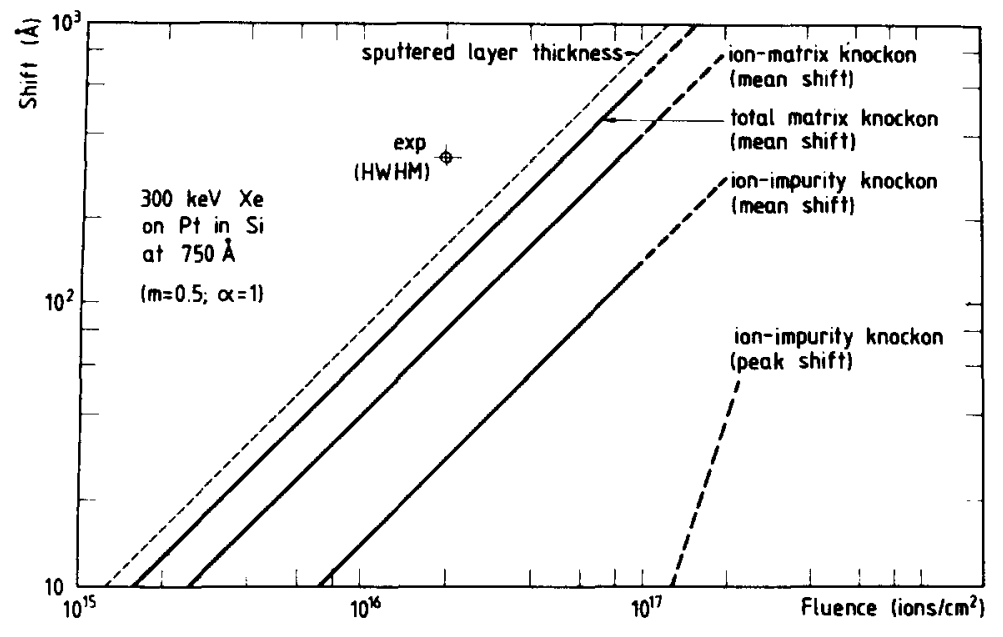

Fig. 5. Marker layer shift for $300 \mathrm{keV} \mathrm{Xe}$ ions incident on a thin Pt layer at $750 \AA$ in Si calculated using Sigmund's recoil implantation model. (from ref. 8)

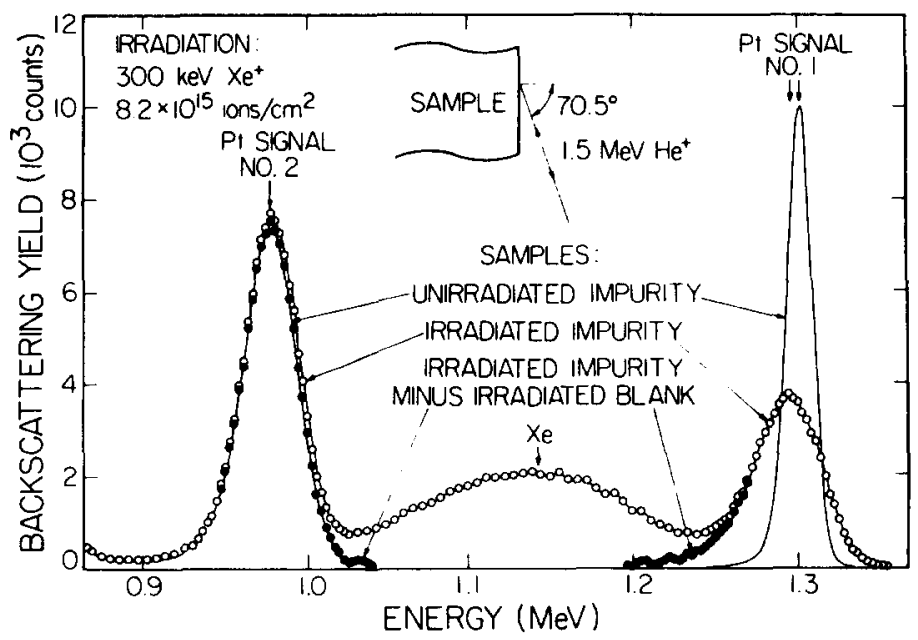

Fig. 6. Backscattering spectra recorded with the unirradiated impurity sample (continuous lines) and the impurity sample irradiated to a fluence of $8.2 \times 10^{15}$ $\mathrm{Xe}$ ions $/ \mathrm{cm}^{2}$ (open circles). (from ref. 13) 
He found a negative shift, that is, a net transport of $\mathrm{Pt}$ away from the surface, in direct contradiction with Sigmund's matrix relocation theory. The linear dependence on fluence was verified in these experiments, Fig. 7. Mantl et al. [14] conducted mixing experiments using dual markers consisting of thin adjacent layers of $\mathrm{Au}$ and $\mathrm{Ni}$ or $\mathrm{Pt}$ and $\mathrm{Ni}$ in either $\mathrm{Si}$ or Al. Mixing with $300 \mathrm{keV}$ $\mathrm{Ar}^{+}$at 20,80 and $300 \mathrm{~K}$ to doses up to $1 \times 10^{17} \mathrm{i} / \mathrm{cm}^{2}$ caused a small $(2-5 \mathrm{~nm})$ negative shift of $\mathrm{Ni}$ to the surface, relative to $\mathrm{Au}$ or $\mathrm{Pt}$. These results support the observations of Paine for net transport of the lighter specie toward the surface. More recently, Auner et al. [15] conducted mixing experiments on $\mathrm{Hf}-\mathrm{Zr}$ bilayers and found that the dominant moving specie is the result of a anisotropic atomic transport which is determined primarily by sample geometry.

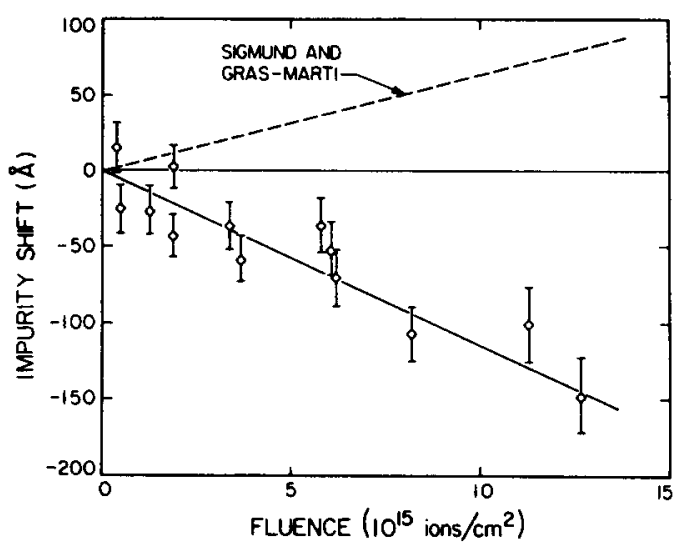

Fig. 7. Shift in the mean position of the Pt layer (diamonds) caused by $300 \mathrm{keV}$ Xe irradiation, plotted against irradiation fluence. Positive shift indicates movement toward the sample surface. The dashed line is the calculation by Sigmund and Gras-Marti [8] for a dilute Pt impurity buried at a depth of $750 \AA$. (from ref. 13)

Rousch et al. [16] conducted Monte Carlo simulations on ion bombarded alloys consisting of 2 components with equal binding energies. They found that there is a preferential movement of the heavier species inward, due primarily to the recoil cascade. Recently, Littmark [17] has conducted theoretical calculations on marker shifts based on transport theory and including a lattice relaxation effect. Calculations for $300 \mathrm{keV} \mathrm{Xe} \mathrm{X}^{+}$bombardment of $\mathrm{Pt}$ markers buried in $\mathrm{Si}$ and 400 
$\mathrm{keV} \mathrm{Xe}^{+}$bombardment of $\mathrm{W}$ markers buried in $\mathrm{Cu}$ at different depths predicted marker shift directions which are in agreement with observations in experiments.

\section{B. Cascade (isotropic, displacement) mixing}

A collision cascade generates a large number of recoils in the low energy range with displacement distances on the order of a few atomic distances. However, because the collision cross section increases with decreasing energy, the number of such events can become extremely large compared to relocation by recoil implantation and so matrix relocation by low energy events can dominate the picture. Although the ion fluence generates an anisotropic distribution of knock-ons, the statistical independence of subsequent events produces nearly isotropic mixing which can be characterized by a random walk of the impurity. As long as the total relocation $\Delta x$ is small enough so that the relocation cross section does not vary significantly over this distance, the mean relocation can be described by [8]:

$$
<\Delta x>=\Phi \int_{z} d \sigma(x, z),
$$

and the mean spread by

$$
\Omega^{2}=\left\langle\left(\Delta x-\langle\Delta x>)^{2}\right\rangle=\Phi \int z^{2} d \sigma(x, z) .\right.
$$

The relocation profile becomes

$$
P(\Delta x)=1 / 2 \pi \int \exp (i k \Delta x-\Phi \sigma(k)) d k
$$

where

$$
\sigma(k)=\int\left(1-e^{-i k z}\right) d \sigma(x, z)
$$

For elastic collisions, the relocation cross section is given by

$$
\mathrm{d} \sigma=\mathrm{dz} \Gamma\left(\mathrm{F}_{\mathrm{D}}(\mathrm{x}) / \mathrm{N}\right) \int\left(\mathrm{dT} / \mathrm{T}^{2}\right)\left(\mathrm{S}_{21}(\mathrm{~T}) / \mathrm{S}_{22}(\mathrm{~T})\right) \int\left(\mathrm{d}^{2} \Omega^{\prime} / 4 \pi\right) \mathrm{F}_{1}\left(\mathrm{~T}, \mathbf{\Omega}^{\prime}, \mathrm{z}\right),
$$


where $S_{21}$ and $S_{22}$ are stopping cross sections for matrix-impurity and matrixmatrix collisions, respectively, $\Gamma$ is a dimensionless parameter, $F_{D}$ is the deposited energy per unit depth and $F_{1}$ is the range distribution of an impurity with initial energy $T$ and recoil direction $\Omega$. This expression can be simplified using a well-defined impurity range relation $[10,11], R_{p}(T)=A T \alpha$ yielding

$$
\mathrm{d} \sigma(x, z)=\Gamma\left(F_{D}(x) / N\right) \xi_{21}[2(\alpha+1)]^{-1} d(z / A)(|z| / A)^{-1-1 / \alpha},
$$

where $\xi_{12}=\mathrm{S}_{12} / \mathrm{S}_{22}$.

For recoils in the $\mathrm{eV}$ range, the relocation profile becomes Gaussian with $\langle\Delta x\rangle=$ 0 ,

$$
\mathrm{P}(\Delta \mathrm{x}) \sim\left(2 \pi \Omega^{2}\right)^{-1 / 2} \exp \left[-(\Delta \mathrm{x}-<\Delta \mathrm{x}>)^{2} / 2 \Omega^{2}\right]
$$

and the spread is given by

$$
\Omega^{2}=1 / 3(1-2 \alpha)^{-1}(\Gamma / N) \Phi F_{D} \xi_{21}\left(R_{c}^{2} / E_{c}\right)=4 D * t,
$$

yielding an effective diffusion coefficient,

$$
D^{*}=1 / 12(1-2 \alpha)^{-1}(\Gamma / N) \phi F_{D} \xi_{21}\left(R_{c}^{2} / E_{c}\right)
$$

where $E_{c}$ is a minimum threshold recoil energy corresponding to a minimum displacement distance, $\mathbf{R}_{\mathrm{c}}=\mathrm{AE}_{\mathrm{c}} \boldsymbol{\alpha}$. A similar expression was also developed by Andersen [18] by assuming that since cascade mixing is isotropic, then the result is a cumulative random-walk-like displacement process which can be characterized by an effective diffusion coefficient $D^{*}$, analagous to that developed in the atomistic model for thermal diffusion,

$$
D^{*}=1 / 6 \lambda^{2} P,
$$

where $\lambda$ is the root-mean-square separation for a vacancy-interstitial pair and $P$ is given by eqn. (2.0). The resulting equation for $\mathrm{D}^{*}$ is

$$
\mathrm{D}^{*}=(1 / 6)\left(0.8 / 2 \mathrm{NE}_{\mathrm{d}}\right)(\mathrm{dE} / \mathrm{dx})_{\mathrm{n}} \lambda^{2} \phi,
$$

which is essentially the same expression developed by Sigmund [8], eqn (3.18).

The effect of cascade mixing is to smear out an originally sharp interface or to broaden a delta function to a Gaussian distribution. For an infinitely thin layer 
of element $B$ in element A, the solution of Fick's second law gives the concentration as a function of position at any time as

$$
C(x, t)=\alpha / 2\left(\pi D^{*} t\right)^{-1 / 2} \exp \left(-x^{2} / 4 D^{*} t\right),
$$

where $\alpha$ is the amount of initial amount of element B. The concentration profile for a pair of initially semi-infinite solids is

$$
C(x, t)=\alpha / 2\left[1+\operatorname{erf}\left(x / 2 \sqrt{D^{*} t}\right)\right]
$$

and for a thin film of thickness a,

$$
C(x, t)=\alpha / 2\left[\operatorname{erf}(x / 2 \sqrt{D t})-\operatorname{erf}\left(x-a / 2 \sqrt{D^{*} t}\right)\right] .
$$

From eqn. (3.17), which relates $D^{*}$ to $\Omega^{2}$, the concentration profile can be determined directly. Note that broadening is proportional to $(\phi t)^{1 / 2}$. In terms of experimentally measured spectra,

$$
\begin{gathered}
\Omega_{\text {total }}^{2}=\Omega_{\text {unirrad }}^{2}+\Omega^{2} \text { mixing, } \\
\Omega^{2} \text { total }=\Omega^{2}{ }_{\text {unirrad }}+4 D^{*} \text { t. }
\end{gathered}
$$

Sigmund [8] calculated the broadening of an initially narrow $\mathrm{Pt}$ layer in $\mathrm{Si}$ as a function of ion fluence for both low energy and high energy cascades, Fig 8 . He found that up to $\Phi \sim 1016$ ions $/ \mathrm{cm}^{2}$, the ion-impurity knock-on profile is determined by single events, while at $\Phi=10^{17}$ ions $/ \mathrm{cm}^{2}$, a multiple relocation profile emerged. At the highest fluence, $10^{17} \mathrm{ions} / \mathrm{cm}^{2}$, high- and low-energy isotropic cascade mixing yield comparable contributions both within and beyond the halfwidth of the distribution.

Many experiments have been conducted to measure the mixing under ion irradiation. They center about either mixing of bilayers or multilayers, or broadening of thin marker layers. Matteson and Nicolet [19] gave a nice review of ion beam mixing experiments using these three geometries. Paine measured the broadening of a $\mathrm{Pt}$ marker layer buried in $\mathrm{Si}$ in the same experiment used to determine the marker shift [13]. Both Sigmund and Gras-Marti [8,20] and Matteson et al. [21,22] predict a linear dependence of $\Omega^{2}$ on $\phi$, as is observed in 
Paine's [13] results, Fig. 9. However, the gradient of the curve is inconsistent with that measured by experiment by about a factor of 2. Matteson et al. [22] conducted mixing experiments on marker layers of $\mathrm{Ni}, \mathrm{Ge}, \mathrm{Pd}, \mathrm{Sn}, \mathrm{Sb}, \mathrm{Pt}$ and $\mathrm{Au}$ in $\mathrm{Si}$ using $\mathrm{Ne}, \mathrm{Ar}, \mathrm{Kr}$ and $\mathrm{Xe}$ ions at $50,110,220$ and $300 \mathrm{keV}$, respectively. The energies were selected to yield equivalent projected ranges and the doses were selected to give approximately equal amounts of mixing using estimates based on the premise that the mixing is approximately proportional to the product of the dose and the nuclear stopping power. Results confirmed that the mixing parameter $\mathrm{Dt}$ is proportional to $\phi$ over a wide range of doses. The data is also consistent with $\mathrm{Dt} / \phi$ being proportional to $(\mathrm{dE} / \mathrm{dx})_{n} / \mathrm{R}_{\mathrm{p}}$, the ratio of the total nuclear energy loss of the mixing ion and its projected range.



Fig. 8. Broadening of the marker layer for $300 \mathrm{keV} \mathrm{Xe}$ ions incident on a thin $\mathrm{Pt}$ layer at $750 \AA$ in $\mathrm{Si}$ (same geometry as in Fig. 7) calculated using Sigmund's relocation model. (from ref. 8)

However, Wang et al. $[23,24]$ have studied the mixing behavior for collisionally similar systems, $\mathrm{Cu}-\mathrm{Au}-\mathrm{Cu}$ and $\mathrm{Cu}-\mathrm{W}-\mathrm{Cu}$ using the same structure for the samples for both systems. It was found that in the case of the $\mathrm{Cu}-\mathrm{Au}$ system, peak broadening is an order of magnitude larger than that in the $\mathrm{Cu}-\mathrm{W}$ system. Other collisionally similar systems, Al-Sb-Al and Al-Ag-Al studied by Picraux et al. [25] showed the mixing rate in the $\mathrm{Al}-\mathrm{Ag}$ system to be 2.6 times that in the $\mathrm{Al}$-Sb system. Other discrepancies in mixing rates have been found and a complete review of mixing experiments has been conducted by Paine and Averback [26]. They found that mixing depends on several parameters. First, 


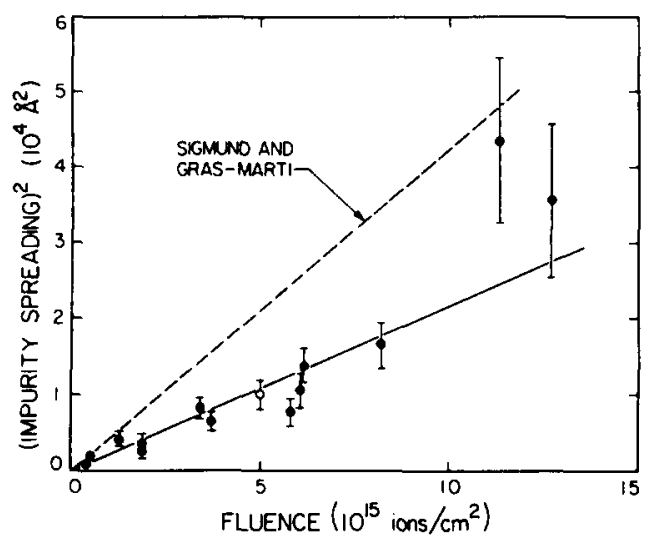

Fig. 9. Variance $\sigma^{2}$ of the irradiation-induced mixing of the $P t$ layer plotted against irradiation fluence (filled circles). The open circles are the measurements by Matteson et al. [21], scaled by the appropriate ratio of values of $F_{D}$ for a layer depth of $500 \AA$. The dashed line is the calculation by Sigmund and Gras-Marti [8]. (from ref. 13)

the profiles in marker layer systems after mixing are almost always Gaussian as expected from theory. For both markers and bilayers, mixing appears to be independent of temperature below $\sim 80 \mathrm{~K}$. The parameter, $\mathrm{Dt}$, does not vary with the irradiation flux $\phi$, but it is linear with fluence, $\Phi$, below $T_{c}$, the characteristic temperature below which thermal processes become negligible. Below $\mathrm{T}_{\mathrm{c}}, \mathrm{Dt}$ generally varies linearly with $F_{D}$. However, $X e$ ions produce a factor of 2 more mixing than $\mathrm{N}, \mathrm{Ne} \mathrm{Ar}$ and $\mathrm{Sb}$ ions in $\mathrm{Ni}(\mathrm{Au})$ marker layer systems. Also, for $\mathrm{Si} / \mathrm{Pt}$ bilayers at low temperatures, mixing efficiencies for heavy ions ( $\mathrm{Kr}$ and $\mathrm{Xe}$ ) were greater than for light ions ( $\mathrm{He}$ and $\mathrm{Ne}$ ) by about a factor of four.

Fu-Zhai and Heng-De [27] used a Monte Carlo code to simulate the transportation process of an incident ion and the cascade of all recoils in an elementary amorphous, bilayered target. They found that the interface mixing was remarkably sensitive to the interface potential (between layers $A$ and $B$ ). Further, primary recoil mixing contributes only $9.3 \%$ of the total mixed Sb atoms in a $\mathrm{Sb} / \mathrm{Si}$ bilayer target, while long range mixing contributes nearly $50 \%$.


the interface.

Paine and Averback [26] have also reviewed the mixing of marker layer efficiency in $\mathrm{Si}$ and $\mathrm{Ge}$, Fig. 10 and $\mathrm{Al}_{2} \mathrm{O}_{3}, \mathrm{SiO}_{2}$ and metals, Fig. 11 as a function of the $z$ of the marker. As shown, the collisional model of Sigmund and Gras-Marti 
[8] cannot explain the large variation of the results with marker species. Such large differences in mixing efficiency between different markers suggest that the chemical nature of the marker must play a role.

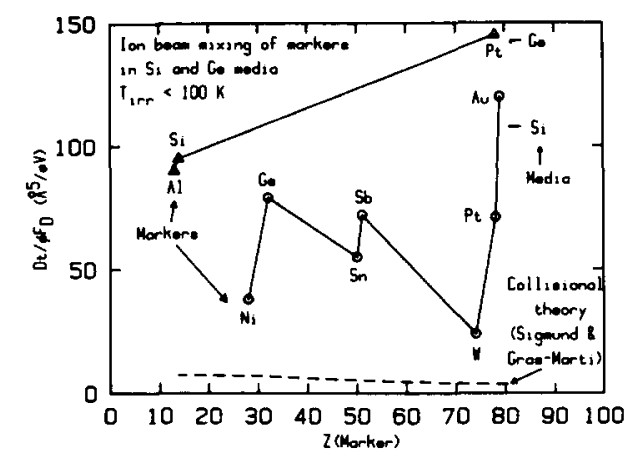

Fig. 10. The efficiency of marker mixing $D t / \phi F_{D}$, plotted as a function of the atomic number of the markers in $\mathrm{Si}$ and $\mathrm{Ge}$ media. The plotted points are the averages of all reported data for irradiations below 100K. The dashed line is calculated from the low energy collision cascade model of Sigmund and Gras-Marti [8]. (from ref. 26)

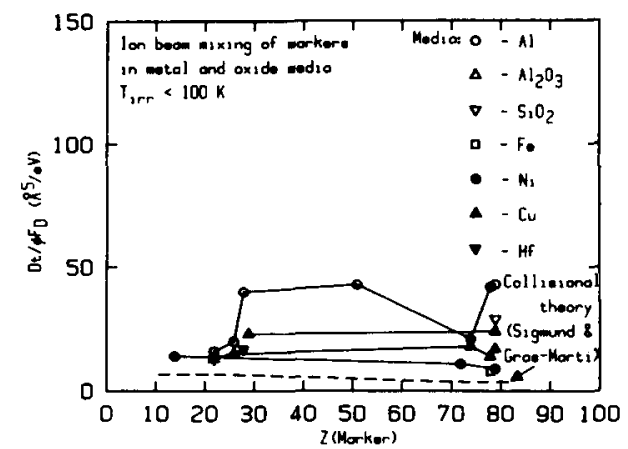

Fig. 11. The efficiency of marker mixing, $\mathrm{Dt} / \phi \mathrm{F}_{\mathrm{D}}$, plotted against the atomic number of the marker for metal and oxide media. Again, the plotted points are the average of all reported data for irradiations below 100K. The dashed line is calculated from the low energy collision cascade model of Sigmund and Gras-Marti [8]. (from ref. 26)

\section{Chemical effects}

Bilayer mixing rates have been found to depend strongly on the nature of the layers as noted earlier in experiments on $\mathrm{Cu}$-Au layers where the mixing was an order of magnitude greater than in $\mathrm{Cu}-\mathrm{W}$ layers [23,24], and in the greater mixing rate (by a factor of 2.6) in $\mathrm{Ag} / \mathrm{Al}$ as compared to $\mathrm{Sb} / \mathrm{Ag}$ [25]. d'Heurle et al. [28] found that the chemical affinity of the elements has a controlling effect on the amount of mixing which is obtained. Minimal mixing is observed with pairs such as $\mathrm{Zr} / \mathrm{Hf}$ or $\mathrm{Pd} / \mathrm{Pt}$, and maximal mixing with pairs such as $\mathrm{Zr} / \mathrm{Pt}$ or Pd/Hf. The results imply that a high chemical affinity between the elements results in high mixing. Cheng et al. [29] also noted a strong chemical effect and was able to show that the mixing rates could be correlated in a linear manner with the liquid alloy heat of mixing of a given metal pair, Figs. 12 and 13 . In 1985, Johnson et al. [30-33] proposed a phenomenological equation to account for the observed chemical effects, based on the presumption that the dominant 
contribution to ion mixing occurs when particle kinetic energies are of order $1 \mathrm{eV}$, and properly accounting for the Kirkendall effect to describe diffusional intermixing in non-ideal solutions.

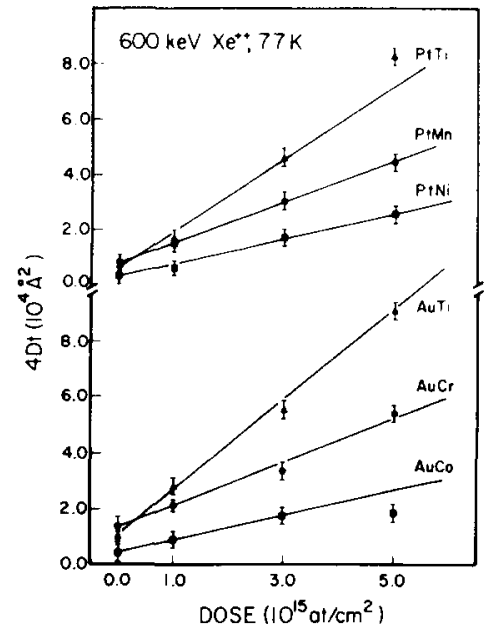

Fig. 12. Variance of interface profile vs dose for selected bilayers irradiated with $600 \mathrm{keV} \mathrm{Xe} \mathrm{Xe}^{++}$at $77 \mathrm{~K}$. (from ref. 29)

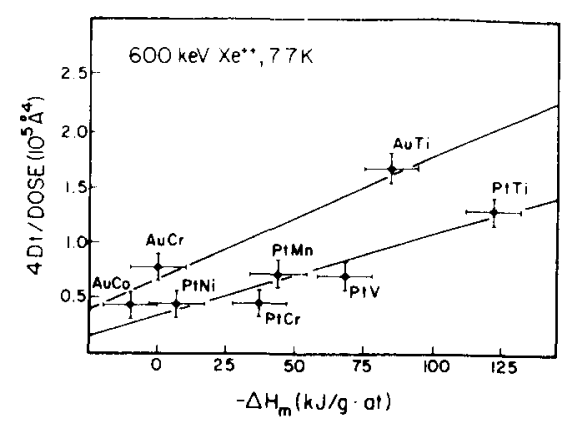

Fig. 13. Correlation between mixing parameter and Miedema's heat of mixing for various bilayers irradiated with $600 \mathrm{keV} \mathrm{Xe}^{++}$at $77 \mathrm{~K}$. (from ref. 29)

Johnson argues that although recoil mixing in the ballistic regime yields a density of displaced atoms at a given distance from the interface which is linear with ion fluence, low energy mixing in a well developed cascade can be described by a random walk or diffusional process $[18,34]$ which will dominate ballistic mixing in well developed cascades [29]. In fact, the measured mixing rates for different pairs of elements can be accounted for by replacing the diffusion coefficient in Fick's law by a modified D' which accounts for the Kirkendall effect and describes diffusional intermixing,

$$
\begin{gathered}
D^{\prime}=D_{0}^{\prime}\left[1-2 \Delta H_{m i x} / k T\right], \\
\text { where } \Delta H_{m i x}=2 \delta C_{A} C_{B}, \\
\text { and } \delta=\left[V_{A B}-\left(V_{A A}+V_{B B}\right) / 2\right],
\end{gathered}
$$

where $V_{A A}, V_{B B}$ and $V_{A B}$ are the potential energies of the interaction of the respective pairs. Physically, this equation says that a random walk will be 
biased when the potential energy depends on the configuration. This equation can be fit to the data [29] to obtain a value of $\mathrm{kT}_{\text {eff }}=1-2 \mathrm{eV}$, indicating that chemical biasing can only contribute to ion mixing when the particle kinetic energies are of order $1 \mathrm{eV}$. Since strong chemical effects have been noted, this implies that the dominant contribution to ion mixing occurs in the $1 \mathrm{eV}$ range!

Johnson [30] postulates that the effective diffusion constant per unit dose rate can be described by an equation of the form

$$
4 D^{\prime} t / \Phi=K_{1} \varepsilon^{2} /\left(\rho^{5 / 3} \Delta H_{\operatorname{coh}^{2}}\right)\left[1+K_{2}\left(\Delta H_{m i x} / \Delta H_{c o h}\right)\right],
$$

which can be used to predict mixing profiles for an arbitrary metal bilayer with well developed cascades in the thermalizing regime, Fig. 14. In this equation, $\varepsilon$ is the energy deposited per unit path length, $\rho$ is the average atomic density of the target, $\Delta H_{\text {coh }}$ is the binding enthalpy per atom and $K_{1}$ and $K_{2}$ are constants. The condition for well developed cascades can be stated in terms of a critical value of $\varepsilon / \Delta H_{\text {coh }} \gg \alpha_{C}$, where $\alpha_{C}$ is the critical value for which ballistic recoil mixing is comparable to diffusive mixing.

Recently, de la Rubia et al. [35] have conducted molecular dynamics (MD) calculations of 3 and $5 \mathrm{keV}$ cascades in $\mathrm{Cu}$ to show that, indeed, the central region of the displacement cascade shows considerable disorder, Fig. 15, and the radial pair-distribution functions, $g(r)$, for the cascade region are quite similar to that of liquid $\mathrm{Cu}$, Fig. 16. The prompt region of the cascade evolution is divided into two subregimes; the ballistic regime or collisional phase in which atomic displacements up to $0.1 \mathrm{ps}$ are well described by ballistic mixing theory, and the thermalizing regime or cooling phase $(t=10 \mathrm{ps})$ during which atomic mixing occurs by a diffusional process. Using these definitions for the respective temporal zones of the cascade, the authors concluded that the majority of the mixing occurs in the region of the melt and is not associated with Frenkel-pair production. They observed that only a relatively small fraction of the atomic mixing takes place up to $0.12 \mathrm{ps}$ (roughly the end of the collisional phase), whereas the vast majority of the mixing occurs during the thermal spike $(t=$ 10ps) and can be accounted for by diffusion in the locally melted region, Fig. 17. Further, energy densities are typically of the order of 1-10 eV/atom and the mean time interval ( $<1.0 \mathrm{ps})$ between collisions in this energy range is small relative to the lifetime of the cascade. These results are consistent with observations of a chemical effect of mixing (which could only occur if mixing was dominant in the eV/atom range) and support Johnson's phenomenological model. The results are supported by those of Webb et al. [36] who showed melting in 5 $\mathrm{keV} \mathrm{Ar}^{+}$bombarded $\mathrm{Cu}$ within $0.2 \mathrm{ps}$. Most recently, Ibe [37] has shown that 
the amount of mixing is equally dependent on the heat of mixing for alloy formation and the intrinsic diffusion coefficients. Hence the present picture of mixing in ion irradiated solids can be summarized by the following:

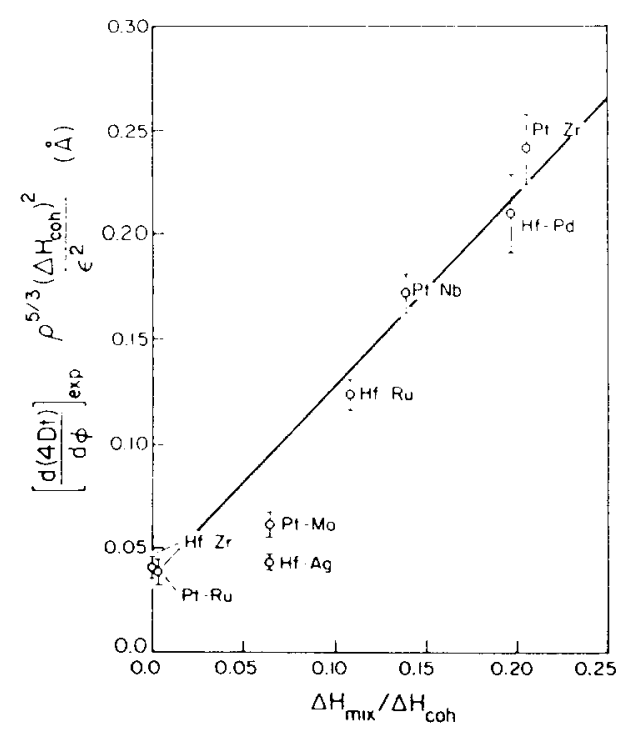

Fig. 14. Correlation between the normalized mixing parameter and $\Delta \mathrm{H}_{\mathrm{mix}} / \Delta \mathrm{H}_{\mathrm{coh}}$ for bilayers irradiated with $600 \mathrm{keV} \mathrm{Xe}^{++}$, and for $\mathrm{K}_{1}=0.034 \AA$ and $\mathrm{K}_{2}=27$. (from ref. 31)



Fig. 16. Radial pair distribution functions, $\mathrm{g}(\mathrm{r})$, corresponding to the disordered zones at $\mathrm{t}=1.10$ and 3.84 ps. Distribution function for liquid $\mathrm{Cu}$ is included for comparison. (ref. 35)

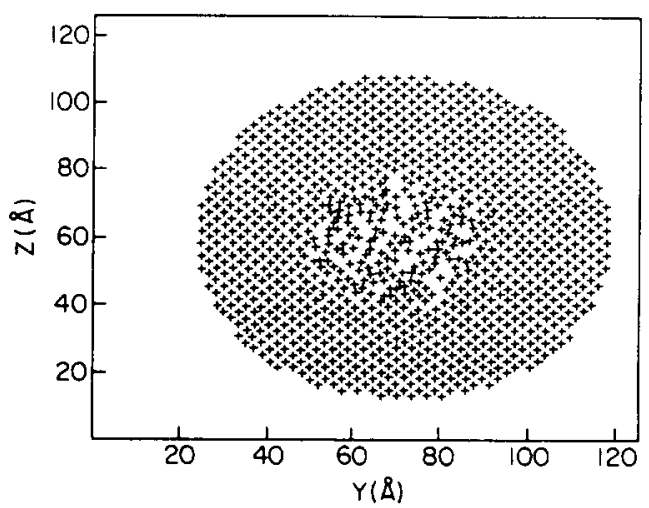

Fig. 15. Locations of atoms in a (100) cross sectional slab of thickness $a_{0} / 2$ through the center of a $5-\mathrm{keV}$ cascade at $t=1.1$ ps. (from ref. 35)

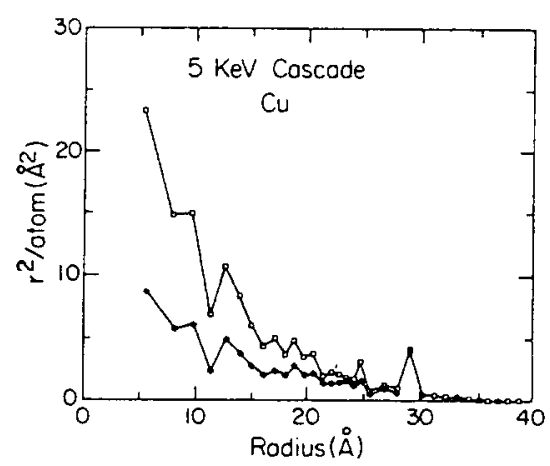

Fig. 17. The integrated diffusion coefficient as a function of distance from the center of the cascade at the end of the collisional phase, $t=0.12 \mathrm{ps}$ (diamonds) and at the end of the cooling phase, $t=10.0 p s$ (squares). (from ref. 35) 
1) The core of the cascade region resembles that of a liquid,

2) mixing in the $1-10 \mathrm{eV} / \mathrm{atom}$ range strongly dominates that at higher energies, and

3) because of 1) and 2), chemical effects can strongly affect the extent of mixing in all systems.

Although this description describes the situation at low temperature, as temperatures increase, radiation enhance diffusion begins to play a role in the observed mixing.

\section{Radiation-enhanced diffusion}

In the absence of radiation, the diffusion of vacancies and interstitials is characterized by a random walk process which is described by an equation of the form

$$
\begin{gathered}
D=D_{0} \exp \left(-\Delta H_{m} / k T\right), \\
D_{0}=\alpha a_{o}{ }^{2} v \exp \left(\left(\Delta S_{t h}+\Delta S_{m}\right) / k\right),
\end{gathered}
$$

where $\alpha$ depends on crystal structure, $a_{o}$ is the lattice parameter, $v$ is the Debye frequency, $\Delta S_{t h, m}$ are the configurational and mixing entropies, respectively, $\Delta H_{m}$ is the defect migration enthalpy, $k$ is Boltzmann's constant and $T$ is the temperature. In the presence of irradiation, the thermally-activated free migration of irradiation-induced vacancy and interstitial defects is known as radiation-enhanced diffusion (RED). Sizmann [38] has written a comprehensive review of RED including dependencies on temperature, dose and dose rate. He notes that diffusion can be significantly enhanced under irradiation by either of two mechanisms: (1) by increasing the concentration of defect species, e.g. vacancies and interstitials which normally provide the means for atom mobility, and (2) by creating other diffusion mechanisms via defect species which are usually not operative. Since the diffusion coefficient is a linear superposition of the various diffusion paths, then

$$
D_{i r r}=f_{v} D_{v} C_{v}+f_{2 v} D_{2 v} C_{2 v}+f_{i} D_{i} C_{i}+\ldots,
$$

where the fs are correlation factors, usually $<1$. Thus the determination of the diffusion coefficient depends on the concentrations of vacancies and interstitials. 
The local change in defect concentration of the various defect species can be written as the net result of the local production rate, reaction rates and divergence of flow. The resulting rate equations are

$$
\begin{gathered}
\mathrm{dC}_{\mathrm{v}} / \mathrm{dt}=\mathrm{K}_{\mathrm{o}}-\mathrm{K}_{\mathrm{iv}} \mathrm{C}_{\mathrm{i}} \mathrm{C}_{\mathrm{v}}+\mathrm{K}_{\mathrm{vs}} \mathrm{C}_{\mathrm{v}} \mathrm{C}_{\mathrm{s}}+\nabla \mathrm{D}_{\mathrm{v}} \nabla \mathrm{C}_{\mathrm{v}}, \\
\mathrm{dC} \mathrm{C}_{\mathrm{i}} / \mathrm{dt}=\mathrm{K}_{\mathrm{o}}-\mathrm{K}_{\mathrm{iv}} \mathrm{C}_{\mathrm{i}} \mathrm{C}_{\mathrm{v}}+\mathrm{K}_{\mathrm{is}} \mathrm{C}_{\mathrm{i}} \mathrm{C}_{\mathrm{s}}+\nabla \mathrm{D}_{\mathrm{i}} \nabla \mathrm{C}_{\mathrm{i}},
\end{gathered}
$$

where $K_{0}$ is the point defect production rate and $K_{i v}, K_{v s}$ and $K_{i s}$ are the rate constants for the reactions indicated by the suffix combination. Note that these equations account for localized sinks by inclusion of the last term in each expression as well as uniformly distributed sinks. Their solution requires the statement of boundary conditions in addition to the initial local concentrations $C_{i, v}(r)$ of the mobile defects $i, v$. However, if the mean defect separation is larger than the mean distance between extended defects, or the sinks can be homogenized without loss of accuracy, then the chemical rate equations become

$$
\begin{gathered}
\mathrm{dC}_{\mathrm{v}} / \mathrm{dt}=\mathrm{K}_{\mathrm{o}}-\mathrm{K}_{\mathrm{iv}} \mathrm{C}_{\mathrm{i}} \mathrm{C}_{\mathrm{v}}+\mathrm{K}_{\mathrm{vs}} \mathrm{C}_{\mathrm{v}} \mathrm{C}_{\mathrm{s}}, \\
\mathrm{d} \mathrm{C}_{\mathrm{i}} / \mathrm{dt}=\mathrm{K}_{\mathrm{o}}-\mathrm{K}_{\mathrm{iv}} \mathrm{C}_{\mathrm{i}} \mathrm{C}_{\mathrm{v}}+\mathrm{K}_{\mathrm{is}} \mathrm{C}_{\mathrm{i}} \mathrm{C}_{\mathrm{s}}, \\
\mathrm{D}_{\mathrm{irr}}=\mathrm{D}_{\mathrm{v}} \mathrm{C}_{\mathrm{v}}+\mathrm{D}_{\mathrm{i}} \mathrm{C}_{\mathrm{i}} .
\end{gathered}
$$

The steady state concentrations are determined by solving eqns (3.33) and (3.34) with both $\mathrm{dC}_{\mathrm{v}} / \mathrm{dt}$ and $\mathrm{dC}_{\mathrm{i}} / \mathrm{dt}$ taken equal to zero, giving,

$$
\begin{aligned}
& \mathrm{C}_{\mathrm{v}}(\mathrm{s})=-\mathrm{K}_{\mathrm{is}} \mathrm{C}_{\mathrm{s}} / 2 \mathrm{~K}_{\mathrm{iv}}+\left[\mathrm{K}_{\mathrm{o}} \mathrm{K}_{\mathrm{is}} / \mathrm{K}_{\mathrm{iv}} \mathrm{K}_{\mathrm{vs}}+\mathrm{K}_{\mathrm{is}}{ }^{2} \mathrm{C}_{\mathrm{s}}{ }^{2} / 4 \mathrm{~K}_{\mathrm{iv}}{ }^{2}\right], \\
& \mathrm{C}_{\mathrm{i}}(\mathrm{s})=-\mathrm{K}_{\mathrm{is}} \mathrm{C}_{\mathrm{s}} / 2 \mathrm{~K}_{\mathrm{iv}}+\left[\mathrm{K}_{\mathrm{o}} \mathrm{K}_{\mathrm{vs}} / \mathrm{K}_{\mathrm{iv}} \mathrm{K}_{\mathrm{is}}+\mathrm{K}_{\mathrm{vs}}{ }^{2} \mathrm{C}_{\mathrm{s}}{ }^{2} / 4 \mathrm{~K}_{\mathrm{iv}}{ }^{2}\right]
\end{aligned}
$$

Note that the steady state concentrations of vacancies and interstitials gives the dependencies on the temperature, sink concentration, total dose and dose rate. In particular, at low temperature and for low to intermediate sink densities, recombination dominates loss to sinks and the vacancy and interstitial concentrations can be approximated by

$$
\begin{aligned}
& \mathrm{C}_{\mathrm{v}}=\left(\mathrm{K}_{\mathrm{o}} \mathrm{K}_{\mathrm{is}} / \mathrm{K}_{\mathrm{iv}} \mathrm{K}_{\mathrm{vs}}\right)^{1 / 2}, \\
& \mathrm{C}_{\mathrm{i}}=\left(\mathrm{K}_{\mathrm{o}} \mathrm{K}_{\mathrm{vs}} / \mathrm{K}_{\mathrm{iv}} \mathrm{K}_{\mathrm{is}}\right)^{1 / 2},
\end{aligned}
$$

giving 


$$
D_{i r r}=\left(\beta K_{0} D_{v} / 4 \pi R_{i v} N\right)^{1 / 2}+\left(\beta K_{0} D_{i} / 4 \pi R_{i v} N\right)^{1 / 2}
$$

where $\beta$ is the fraction of $v-i$ pairs still present in the delayed regime. This quantity is proportional to the product of dose rate and diffusivity to the $1 / 2$ power. At high temperatures, the steady state concentrations of vacancies and interstitials is given by,

Given that

$$
\begin{gathered}
\mathrm{C}_{\mathrm{v}}=\mathrm{K}_{\mathrm{o}} / \mathrm{K}_{\mathrm{vs}} \mathrm{C}_{\mathrm{s}}, \\
\mathrm{C}_{\mathrm{i}}=\mathrm{K}_{\mathrm{o}} / \mathrm{K}_{\mathrm{is}} \mathrm{C}_{\mathrm{s}} .
\end{gathered}
$$

$$
\mathrm{C}_{\mathrm{s}} \sim \pi^{2} / \mathrm{L}^{2}\left(\Omega / 4 \pi \mathrm{R}_{\mathrm{vs}, \text { is }}\right)
$$

then the diffusion coefficient is

$$
\mathrm{D}_{\mathrm{irr}}=2 \beta \mathrm{K}_{\mathrm{o}} \mathrm{L}^{2} / \pi^{2},
$$

which is directly proportional to the dose rate and the square of the mean distance $L$ between extended sinks. Figure 18 shows the steady state defect concentrations for an irradiated solid at high and low temperatures for high and low dislocation densities [39]. Figure 19 shows the resulting radiation-enhanced diffusion coefficient in a $\mathrm{Ni}$ foil due to ion bombardment at typical dose rates.

In traversing from low to high temperature, ion beam mixing shows an increasingly pronounced dependence on the sample temperature. Matteson [34] found that below $300 \mathrm{~K}$, broadening of $\mathrm{Ni}, \mathrm{Ge}, \mathrm{Pt}$ and $\mathrm{Au}$ in $\mathrm{Si}$ by $200 \mathrm{keV} \mathrm{Kr^{+ }}$ or $300 \mathrm{keV} \mathrm{Xe}^{+}$was temperature independent up to $300 \mathrm{~K}$ and up to $523 \mathrm{~K}$ for $\mathrm{Sn}$ and $\mathrm{Sb}$. However, he also showed [40] that in $\mathrm{Nb}-\mathrm{Si}$, mixing became strongly temperature dependent above $\sim 600 \mathrm{~K}$, Fig. 20 . The temperature at which mixing becomes strongly dependent on temperature is called the critical temperature, $\mathbf{T}_{\mathbf{c}}$. It is also defined as the narrow temperature range which separates the temperature-independent region from the Arrhenius-type radiation-enhanced diffusion region, or by the intersection of the high- and low-temperature asymptotes. Various authors have used the occurrence of $T_{c}$ to deduce the specie responsible for the observed mixing behavior. Cheng et al. [42] describes the behavior of the amount of mixing by an effective diffusion coefficient of the form

$$
D=D_{c}+D_{\text {irr }} \exp (-Q / k T)
$$




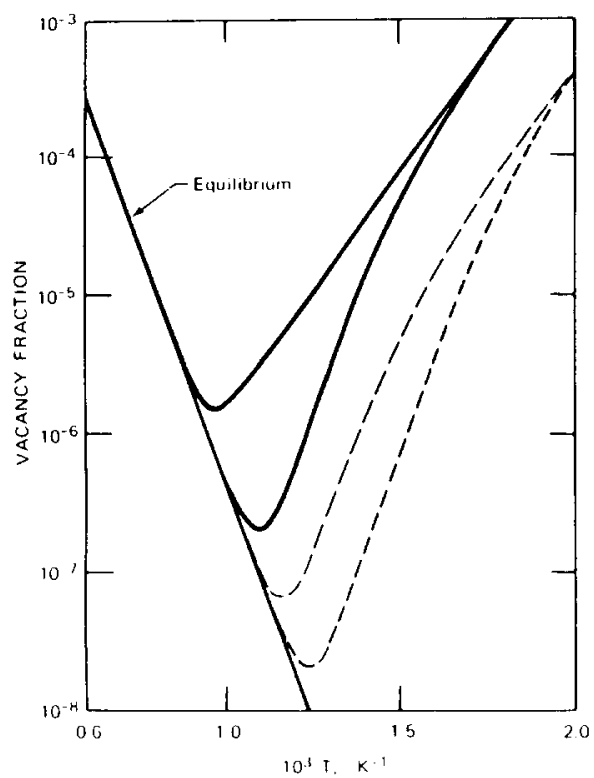

(a)

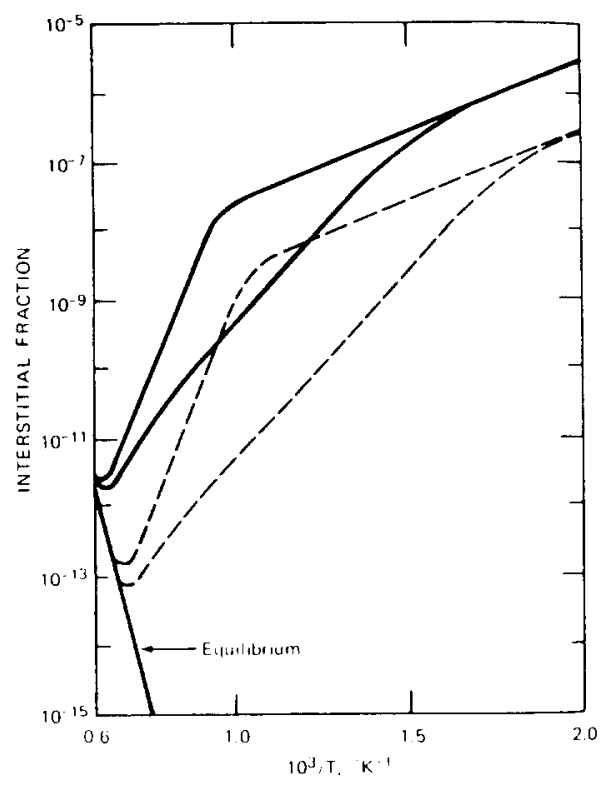

(b)

Fig. 18. Steady-state point-defect concentrations in an irradiated solid at a high defect production rate (solid line) and at a low defect production rate (dashed line). The upper and lower curves for each defect production rate represent small and large dislocation densities, respectively. (from ref. 39)

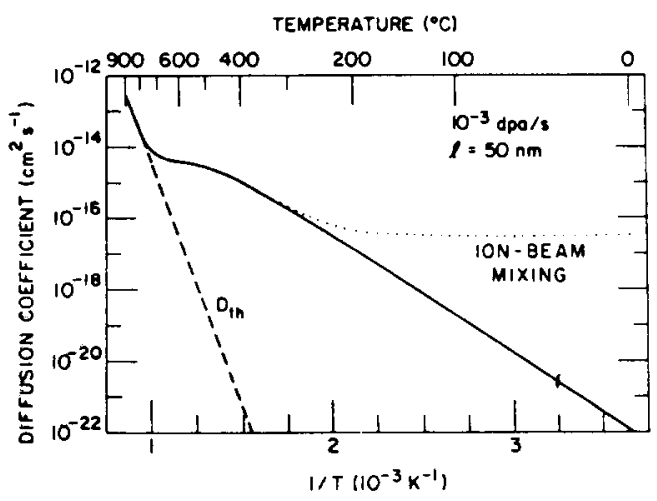

Fig. 19. The mean radiation-enhanced diffusion coefficient in a $500 \AA$ thick foil of $\mathrm{Ni}$ at a damage rate typical of ion bombardment, calculated from the analytical solution to the rate equations. A sink density of $1010 / \mathrm{cm} 2$ was assumed. The curve shown represents a somewhat idealized picture, in that saturation of the vacancy concentration and time-dependence of the defect concentrations at low temperature have been neglected. The thermal diffusion coefficient and the diffusion coefficient due to ballistic mixing are also shown for comparison. (from ref. 41) 


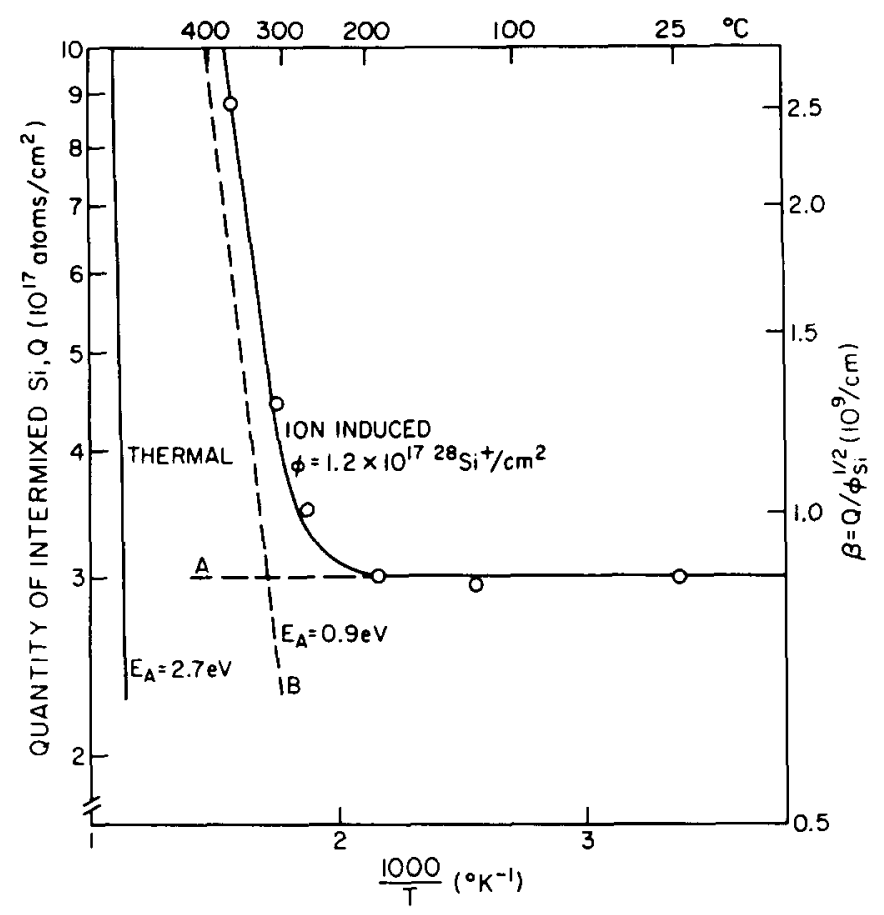

Fig. 20. Logarithm of the quantity of intermixed silicon versus reciprocal temperature for a fluence of $1.2 \times 10^{17} 28 \mathrm{Si}^{+} / \mathrm{cm}^{2}$. The solid line is a fit to the data points of the sum of a temperature independent part (dotted line A) and a thermally activated part (dotted line $B$ ) with an activation energy $\mathrm{E}_{\mathrm{A}} \sim 0.9 \mathrm{eV}$. The quantity of intermixed silicon which would be produced by thermal silicide growth without irradiation in the same time interval is included in the figure for comparison. The scale on the right is for coefficient $\beta$ where $Q=\beta \phi^{1 / 2}$. (from ref. 40)

where the first term on the right-hand side is due to cascade mixing and is temperature independent, and the second term is due to radiation-enhanced diffusion and has an Arrhenius-type temperature dependence, where $Q$ is an apparent activation energy. At a temperature near $T_{c}$, the two terms on the right of eq. (3.45) contribute equally and

$$
T_{c}=(1 / k)\left[\ln \left(D_{i r r} / D_{c}\right)\right]^{-1} Q .
$$


Assuming the existence of a scaling relationship between $Q$ and the cohesive energy of the matrix, $E_{c o h}$, i.e., $Q=S E_{c o h}$, then eqn (3.46) can be written as

$$
T_{c}=(S / k)\left[\ln \left(D_{i r r} / D_{c}\right)\right]^{-1} E_{c o h} .
$$

A correlation between $T_{c}$ and $E_{c o h}$ is given in Fig. 21 for 10 binary systems, verifying the predicted linear relationship. Cheng estimated that the average value of $\ln \left(D_{i r r} / D_{c}\right) \sim 11.6$, and from the slope of the line determined that $S \sim 0.1$ and $Q \sim 0.1 E_{c o h}$. Since $E_{m}{ }^{v}=0.24 E_{c o h}$ by the same scaling relationship, $\mathrm{Q} \sim 0.12 \mathrm{E}_{\mathrm{coh}}$. Cheng concludes that this value for the activation energy (half of the vacancy migration energy) is consistent with a model based on a vacancy mechanism for radiation enhanced diffusion [43,44]. This result is also consistent with the work of Sizmann [38] which shows that at steady state, the activation enthalpy for $D_{i r r}$ is $0.5 \mathrm{H}_{\mathrm{m}}{ }^{\mathrm{v}}$. This yields a value of $1.03 \mathrm{eV}$ for the vacancy activation enthalpy in nickel which is consistent with the assumption that in Stage III vacancies are mobile.

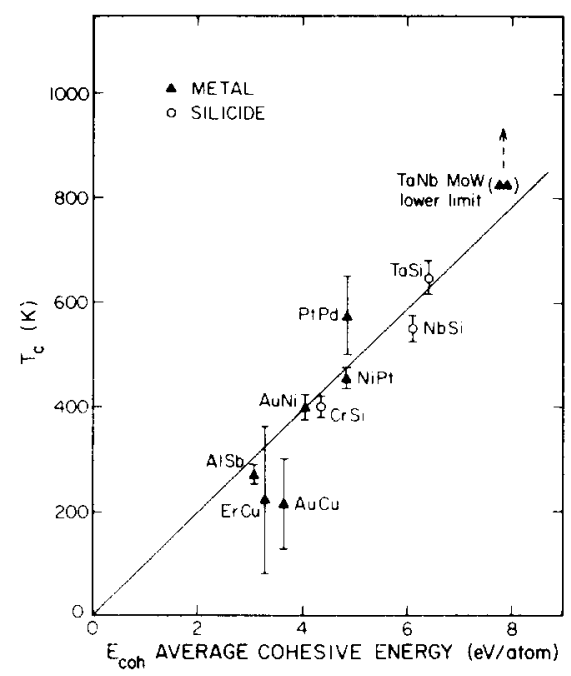

Fig. 21. Correlation between the cohesive energy of the system and the critical temperature $T_{c}$ at which radiation-enhanced diffusion becomes dominant. (from ref. 42)

However, Rauschenbach has observed that $T_{c}$ is often well below stage III and along with Peak and Averback [45] has concluded that because of its high migration enthalpy "normal" vacancy migration does not contribute significantly 
to mixing. Rauschenbach [46] has proposed that the observed $T_{c}$ is in fact, due to the migration of mixed dumb-bells via orthogonal jumps into nearestneighbor positions. From this he has developed an expression for the characteristic temperature $T_{c}$ by equating the diffusivity due to vacancies and interstitials at $\mathrm{T}<\mathrm{T}_{\mathrm{c}}$ to that due to diffusivity by mixed dumb-bell migration at $\mathrm{T}>\mathrm{T}_{\mathrm{c}}$ :

$$
T_{c}=(1 / k)-H_{m} v /\left[\ln \left(K_{0} / C_{s}^{2} \beta\right)-\ln \left(2 v_{v} / v_{c} \alpha_{c v}\right)\right]
$$

where $v_{v, c}$ is the Debye frequency for vacancies and complexes, respectively, and $\alpha_{c v}$ is the recombination coefficient. The calculated values of $T_{c}$ were compared to measured temperatures, $T_{c} \exp$ for 5 marker layer systems and 8 bilayer systems with considerable success.

The observation of low onset temperatures for enhanced mass transport in ion-beam mixed systems has been noted by Rehn and Okamoto [47]. The authors have suggest that enhancement in mixing at temperatures too low to be due to the free migration of irradiation-induced vacancy and interstitial defects may be due to intra- or inter-cascade mechanisms. This can be confirmed by observing the dose rate dependence of $D$.

\section{E. Radiation-induced segregation}

The first experimental observation of radiation-induced segregation was made in 1974 by Okamoto and Wiedersich [48]. Since that time, a complete theory for the mechanism of RIS has been developed [49-58] and considerable experimental evidence exists [59-70] to support the theory. RIS can be classified as non-equilibrium segregation which is driven by kinetic processes rather than by thermodynamic forces as in equilibrium segregation. Defect migration can drive alloy microstructures either toward or away from equilibrium. The nonequilibrium path is driven by radiation-induced segregation, which is caused by the preferential transport of certain alloying components via persistent defect fluxes generated during irradiation. Subsequent annealing at the same temperature in the absence of irradiation will cause radiation-induced segregation effects to diminish. In contrast, radiation-enhanced diffusion results primarily from the random migration of the excess defects generated during irradiation and hence, drives the system toward equilibrium.

Ion irradiation produces atomic displacements and hence, point defects, in solids along the length of the ion track. At high temperatures, these defects are 
mobile and are eliminated by mutual recombination or annihilation at sinks. If either the production, annihilation or both are spatially inhomogeneous, net defect fluxes will be induced. Since the motion of defects requires motion of the atoms, e.g., vacancies exchange sites with neighboring atoms and interstitials jump to neighboring interstices, defects will migrate preferentially by the motions of atoms of one or more alloying elements. Thus, a preferential coupling exists between defect fluxes and fluxes of certain alloying elements [58].

In order for radiation-induced segregation to occur, two conditions must exist [58]: (1) a flux of defects into or out of certain spatial regions that persist in time and (2) a preferential coupling of certain alloying elements to these fluxes. This combination induces and maintains local concentration gradients that will decay in the absence of defect fluxes. As a consequence, defect fluxes will preferentially transport solute atoms into or out of local regions, causing segregation. An important feature of defect-flux driven segregation is that solute redistribution occurs regardless of the initial distribution of solute. This redistribution has been studied most frequently in alloys in which the components were homogeneously distributed throughout the material before irradiation. However, significant effects have also been observed in the depth distribution of implanted solutes [55] as well as in the distribution of solute atoms introduced by ion-beam mixing of a thin surface layer [58].

As discussed by Wiedersich [58], the origins of persistent defect fluxes are many. The most obvious cause for a defect flux into a spatial region is local elimination of excess defects at sinks such as voids, dislocations, grain boundaries, and surfaces. A less prominent cause for persistent defect fluxes is defect trapping at local inhomogeneities such as solute clusters and coherent interfaces that cannot act as independent defect sinks, but increase recombination of vacancies and interstitials by virtue of trapping. Persistent defect fluxes can also arise from non-uniform defect production. Since defect production rates depend on composition, structure and bonding of the material via the threshold displacement energy, defect fluxes may persist between adjacent phases of different compositions and/or different structures. Finally, non-uniform defect production is also a source of persistent defect fluxes. During ion implantation, the defect production rate varies with the depth of the bombarding particle, increasing to a peak at a location that is slightly shallower than that of the implanted ion distribution, and then falling to zero just beyond the end of range. The damage rate at the peak of the damage distribution can be over an order of magnitude greater than that in the flat portion of the profile near the surface. All of these inhomogeneities are sources for persistent defect fluxes which contribute to radiation-induced segregation. 
(i) Segregation mechanisms. Preferential coupling between alloying components and the defect fluxes can occur by two mechanisms: inverse Kirkendall effects and by the formation of mobile defect-solute complexes. These two mechanisms couple a net flux of solute atoms to the defect fluxes, causing disproportionate mass transport into and out of local regions, i.e., segregation. Significant defect fluxes are produced during irradiation only when both types of defects, i.e., vacancy- and interstitial-types are mobile. Otherwise recombination dominates and little long-range mass transport occurs. The temperature range in which defect-flux driven segregation can produce redistribution of alloying components over significant distances is about 0.3 to 0.5 of the absolute melting temperature.

\section{(a) Inverse Kirkendall effect}

Anthony [71] suggested that under irradiation, an "inverse" Kirkendall effect can occur. Recall the Kirkendall effect in which a composition gradient can induce a net flux of defects across a "marker" plane in an alloy. In the "inverse" Kirkendall effect, defect fluxes would induce compositional gradients in an initially homogeneous alloy [72]. Under irradiation, both vacancy and interstitial defects can produce inverse Kirkendall effects, Fig. 22 As shown in Fig. 22a, a vacancy gradient near a sink in a binary alloy composed of elements $A$ and $B$, generates a vacancy flux, toward the sink, which induces an atom flux $\left(J_{A}{ }^{v}+J_{B} v\right)$ of equal magnitude in the opposite direction, where $J_{A} v$ and $J_{B} v$ are the fluxes of $A$ and $B$ atoms, respectively. Since $J_{A} v$ and $J_{B} v$ transport $A$ and $B$ atoms in amounts proportional to their local atom fractions, $C_{A}$ and $C_{B}$, and to their partial diffusion coefficients, $D_{A} v$ and $D_{B} v$, it follows that the alloy composition around the sink does not change when $D_{A} v=D_{B} v$. However, if $D_{A}^{v}=/ D_{B} v$, the flux of the faster diffusing component away from the sink will be proportionately greater than its concentration in the alloy. Therefore, the inverse Kirkendall effect induced by a vacancy flux will always cause depletion, at a sink of the faster diffusing component.

The same process holds for an interstitial flux. However, because the interstitial flux and the complementary atom fluxes, $J_{A} i$ and $J_{B}{ }^{i}$, move in the same direction, Fig. 22b, any difference in the partial diffusion coefficients of the $A$ and $B$ atoms via interstitials, i.e. $D_{A}^{i} \neq D_{B}^{i}$ will result in the preferential transport of the faster diffusing component toward the sink. Therefore, depending on the relative magnitudes of the ratios $D_{A} v / D_{B} v$ and $D_{A} i / D_{B}{ }^{i}$, the two inverse Kirkendall effects may aid or oppose each other in causing solute segregation near a sink. 



Fig. 22. Schematic illustration of inverse Kirkendall effects induced by (a) vacancy flux, and (b) interstitial flux. (from ref. 54)

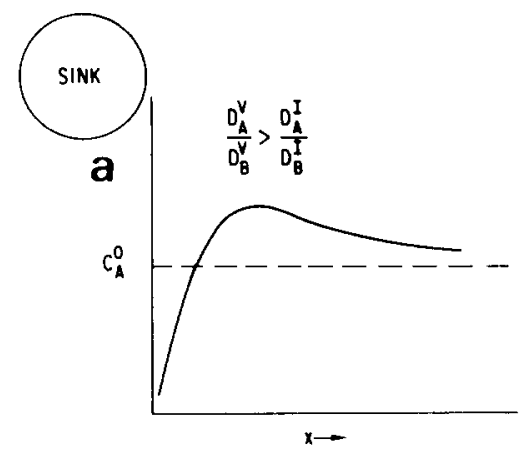

Fig. 23. Effect of partial diffusion coefficients on the depth distribution of A atoms. (from ref. 54)

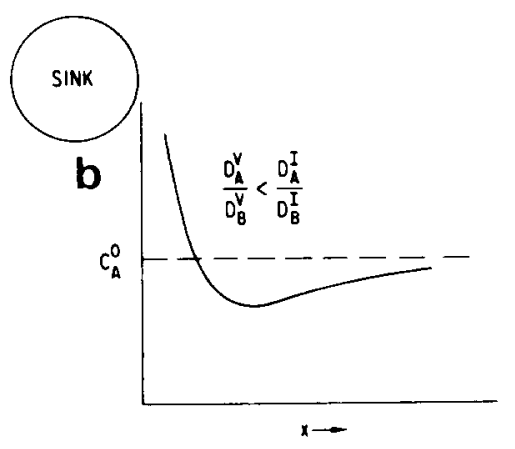


A simple treatment of RIS, proposed by Wiedersich et al. [56], is based on the concept of partitioning the defect fluxes into those occurring by exchange with the various alloy components and the atom fluxes, into those taking place via vacancies and interstitials. In a binary alloy $A B$, the fluxes of atoms, $J_{A}$ and $J_{B}$, and those defects, $J_{v}$ and $J_{i}$, can be expressed in terms of the concentration gradients of all species present as [57]

$$
\begin{aligned}
& \Omega J_{A}=-\left(D_{A}^{v}+D_{A}{ }^{i}\right) \nabla C_{A}+D_{v}^{A} \nabla C_{v}-D_{i}^{A} \nabla C_{i}, \\
& \Omega J_{B}=-\left(D_{B}^{v}+D_{B}^{i}\right) \nabla C_{B}+D_{v}{ }^{B} \nabla C_{v}-D_{i}^{B} \nabla C_{i}, \\
& \Omega J_{v}=-\left(D_{v}^{A}+D_{v}^{B}\right) \nabla C_{v}+D_{A}{ }^{v} \nabla C_{A}+D_{B} v \nabla C_{B}, \\
& \Omega J_{i}=-\left(D_{i}^{A}+D_{i}^{B}\right) \nabla C_{i}-D_{A}^{i} \nabla C_{A}-D_{B}{ }^{i} \nabla C_{B},
\end{aligned}
$$

where $\Omega$ is the average atomic volume and $D_{A} v, D_{A} i, D_{v} A, D_{i} A$ are the partial diffusion coefficients defined so that the subscript indicates the diffusing specie, and the superscript the complementary species via which the diffusion occurs. For example, $D_{A} v$ and $D_{A} i$ are the partial diffusion coefficients of A-atoms migrating via vacancies and interstitials, respectively, and $D_{v} A$ and $D_{i} A$ are the partial diffusion coefficients of vacancies and interstitials, respectively, migrating via $\mathrm{A}$-atoms.

In a concentrated binary alloy, Wiedersich et al. [56] derived the following relation between the steady-state concentration gradient for the A component and the vacancy concentration gradient:

$$
\nabla C_{A}=(1 / \alpha) D_{i}^{B} D_{i}^{A} /\left(D_{i}{ }^{B} D_{A}^{i r r}+D_{i}{ }^{A} D_{B}^{i r r}\right)\left(D_{A}^{v} / D_{B} v-D_{A}^{i} / D_{B}^{i}\right) \nabla C_{v},
$$

$D_{A}{ }^{i r r}$ and $D_{B}$ irr are the total radiation-enhanced diffusion coefficients for the $A$ and $B$ atoms and $\alpha$ is a thermodynamic factor which deviates from unity for non-ideal solutions. The two cases of interest predicted by eqn (3.53) are illustrated in Fig. 23. Segregation of A away from the sink occurs when the preferential transport of $A$ atoms via vacancies exceeds that via interstitials. Conversely, enrichment of $A$ atoms at the sink occurs when the preferential transport of $\mathrm{A}$ atoms by interstitials exceeds that via vacancies. The enrichment of $A$ at the sink is maximized when $A$ atoms diffuse exclusively via interstitials and $B$ atoms via vacancies. 


\section{(b) Defect-solute complexes}

In addition to the inverse Kirkendall effect caused by the different rates at which defects exchange with atoms of different elements, defect-flux driven segregation can also result from the formation of bound defect-solute complexes [58]. An attractive interaction between point defects and solute atoms leads to a preferential association, or defect-solute complex formation, which generally affects not only the defect mobility but also the diffusion of substitutional solute atoms. For example, a positive (attractive) binding energy between vacancies and solute atoms increases the probability of a vacancy occupying a nearestneighbor site of a solute. Therefore, even in the absence of any changes in the vacancy jump frequencies, binding affects the diffusivity of the solute atoms differently than that of the solvent atoms. Similarly, formation of soluteinterstitial complexes will alter the diffusivity of the solute atoms via interstitials. Furthermore, the defect jump frequencies will generally depend on the proximity of solute atoms and their participation in the jump process. Hence, effects of solute atoms on defect jump frequencies, and preferential solutedefect association due to binding, cause a coupling between defect fluxes and solute fluxes.

These defect-solute complexes are especially important for segregation in dilute alloys and have been extensively discussed by Johnson and Lam [49]. Defect-solute complexes can be considered to be mobile when the following relationship is satisfied:

$$
E_{b}^{d-s}+E_{m}^{d}>E_{m}^{d-s},
$$

that is, when the sum of the defect-solute binding energy, $E_{b} d-s$, and the defect migration energy, $E_{m}{ }^{d}$, exceeds the migration energy of the complex, $E_{m} d-s$. Complexes that satisfy this condition may be regarded as distinct entities that can migrate through the crystalline lattices. Since they flow in the same direction as the defect fluxes, both interstitial- and vacancy-type mobile complexes will tend to sweep solute toward sinks in initially homogeneous alloys.

Defect-solute interactions are less effective at high temperatures and so are expected to dominate at low temperatures while inverse Kirkendall effects may dominate at high temperatures [66]. In the absence of interstitial-solute interactions, solute enrichment at sinks can occur at low temperatures via vacancy-solute complexes, and solute depletion at high temperatures due to the vacancy-induced inverse Kirkendall effect. Calculations by Johnson and Lam [49] show that binding energies of $\geq 0.2 \mathrm{eV}$ are required for mobile defect-solute 
complexes to produce significant segregation. Only a few examples of vacancysolute binding energies of this magnitude have been reported in the literature, but several well-documented examples of large $(>0.5 \mathrm{eV})$ interstitial-solute binding energies exist [73]. As discussed by Okamoto and Wiedersich [48], segregation by mobile interstitial-solute complexes is expected to be especially important for undersize solutes since smaller atoms can be more easily be accommodated in interstitial sites.

The preferential participation of $\mathrm{A}$ atoms in the interstitial population can be accounted for by incorporating into the diffusivity coefficients, factors that represent the fractions of $A$ - and B-interstitials [56]:

$$
\begin{gathered}
\mathrm{C}_{A}^{\mathrm{i}}=\mathrm{C}_{\mathrm{i}} \mathrm{C}_{\mathrm{A}} \exp \left(\mathrm{H}_{\mathrm{Ai}} \mathrm{b} / \mathrm{kT}\right) /\left[\mathrm{C}_{\mathrm{A}} \exp \left(\mathrm{H}_{\mathrm{Ai}} \mathrm{b} / \mathrm{kT}\right)+\mathrm{C}_{B}\right], \\
\mathrm{C}_{B}{ }^{\mathrm{i}}=\mathrm{C}_{\mathrm{i}} \mathrm{C}_{\mathrm{B}} /\left[\mathrm{C}_{\mathrm{A}} \exp \left(\mathrm{H}_{\mathrm{Ai}} \mathrm{b} / \mathrm{kT}\right)+\mathrm{C}_{B}\right]
\end{gathered}
$$

where $k$ is the Boltzmann constant, $T$ is the absolute temperature, and $\mathrm{H}_{\mathrm{Ai}} \mathrm{b}^{\mathrm{b}}$ is the energy gained by converting a B-interstitial into an A-interstitial.

However, in order to account for atom transport by tightly-bound, mobile A atom-vacancy ( $\mathrm{vA}$ ) complexes in dilute alloys, additional terms derived from the contribution of the complex flux $\mathrm{J}_{\mathrm{vA}}=-\mathrm{D}_{\mathrm{vA}} \nabla \mathrm{C}_{\mathrm{vA}}$ must be included in eqns (3.49) and (3.51) for $\mathrm{J}_{\mathrm{v}}, \mathrm{J}_{\mathrm{A}}$ and $\mathrm{J}_{\mathrm{B}}$ :

$$
\begin{gathered}
\Omega J_{v}=\left[\left(D_{A} v^{v}-D_{B} v\right) \alpha-K_{v A} D_{v A} C_{v}\right] \nabla C_{A}-\left(D_{v}+K_{v A} D_{v A} C_{A}\right) \nabla C_{v}, \\
\Omega J_{A}=-\left[\alpha D_{A}+\left(1-2 C_{A}\right) K_{v A} D_{v A} C_{v}\right] \nabla C_{A}-D_{i} A \nabla C_{i}+\left[D_{v} A_{-}\left(1-2 C_{A}\right) K_{v A} D_{v A} C_{A}\right] \nabla C_{v}, \\
\Omega J_{B}=\left[\alpha D_{B}+2 C_{B} K_{v A} D_{v A} C_{v}\right] \nabla C_{B}-D_{i} B^{3} C_{i}+\left[D_{v} B+2 C_{B}{ }^{2} D_{v A} D_{v A}\right] \nabla C_{v},
\end{gathered}
$$

where $D_{v A}$ is the diffusion coefficient of the vA complex, $D_{v A}=\lambda^{2} v_{v A} c / 6\left(n_{v A} c\right.$ being the complex jump frequency), and $\mathrm{K}_{\mathrm{vA}}=12 \exp \left(\mathrm{H}_{\mathrm{vA}} \mathrm{b} / \mathrm{kT}\right)$ is the rate constant for the formation of $v A$ complexes in equilibrium $\left(\mathrm{H}_{\mathrm{vA}}{ }^{\mathrm{b}}\right.$ being the complex binding energy).

The idea of tightly bound solute-defect complexes migrating as distinct entities loses its well-defined meaning and its usefulness when the concentration of the solute exceeds a few atom percent [58]. For example, vacancies in concentrated alloys will frequently have more than one solute atom as nearest neighbors. Therefore, a multitude of vacancy-solute complexes containing different numbers of solute atoms, many with several distinguishable configurations, would have to be considered. Furthermore, at high solute 
concentrations, a vacancy jump may re-form a vacancy complex similar to that existing prior to the jump, and 'complex migration' may occur without a corresponding solute flux. Therefore, Weidersich et al. [56] used the formalism described in section 3.E.i to arrive at eqn. (3.53) for the relation between concentration gradients of solute and defects in concentrated alloys.

With regard to interstitials, however, there exists both experimental $[54,74,75]$ and theoretical evidence [76] which supports the formation of tightly bound solute-interstitial complexes for undersized solutes, in both the dilute and concentrated limits, which migrate as solute interstitials. Hence, Wiedersich's model for segregation appears well suited to describe alloy systems with significant atomic size differences, including the limit in which the undersized component is present in dilute solution. The origin of the size effect follows.

(ii) Solute size effect. The size difference between solute and solvent atoms plays a strong role in the magnitude and direction of radiation-induced segregation through the reduction of the strain energy stored in the lattice [51]. This provides the driving force for the undersize solute substitutional atoms to preferentially exchange with solvent atoms in interstitial positions, whereas oversize solute atoms will tend to remain on, or return to, substitutional sites. The same strain-energy considerations will drive vacancies to preferentially exchange with oversize solute atoms. During irradiation at elevated temperature, the fraction of undersize solute atoms migrating as interstitials, or of oversize solute atoms migrating against the vacancy flux, may greatly exceed the fraction of solute in the alloy. Such a disproportionate participation of misfitting solute atoms in the defect fluxes to sinks will cause a redistribution of solute, which will produce an enrichment of undersize solute and a depletion of oversize solute near defect sinks. Since the surface of an irradiated solid serves as an unsaturable link for both defect types, concentration gradients will be created near the surface of alloys that contain misfitting solute atoms during irradiation at appropriate temperatures.

Irradiation of solid solution binary alloys of 1 at\% $\mathrm{Al}, \mathrm{Ti}, \mathrm{Mo}$ and $\mathrm{Si}$ in $\mathrm{Ni}$ with 3.5 $\mathrm{MeV} \mathrm{Ni}{ }^{+}$ions produced sharp concentration gradients near the irradiated surface in all the alloys [51]. The three oversize solutes, $\mathrm{Al}, \mathrm{Ti}$ and Mo with misfits of $+0.05,+0.10,+0.12$ (where misfit is defined as the fractional change in lattice parameter of a solid-solution alloy produced per atom fraction of solute, $(\Delta \mathrm{a} / \mathrm{a}\} / \mathrm{c})$, all exhibited depletion from the irradiated surface and an enriched region at intermediate depths, Fig. 24a-c. The undersize Si, however, shows enrichment at the irradiated surface followed by depletion at intermediate 
depths, Fig. 24d. In fact, Table 2.1 shows some 26 binary alloys in which RIS has been observed along with the corresponding volume misfit [61]. As shown, there are only three discrepancies with theoretical predictions.

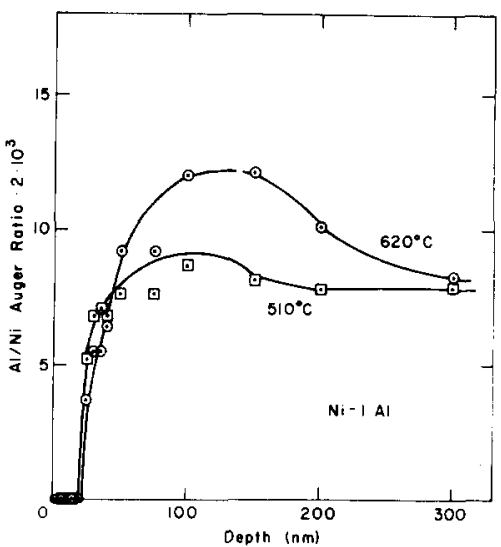

(a)

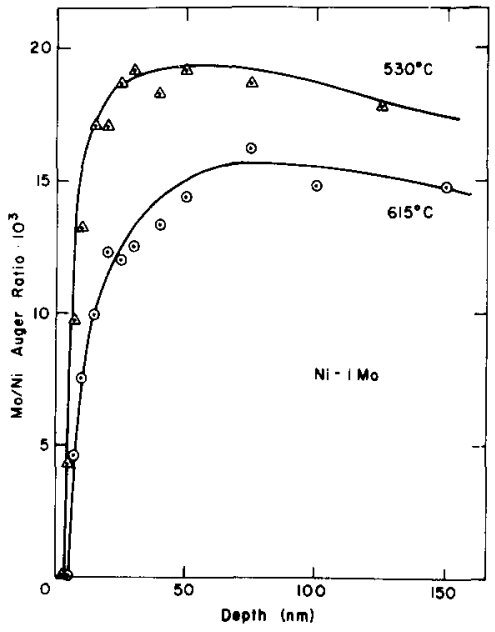

(c)

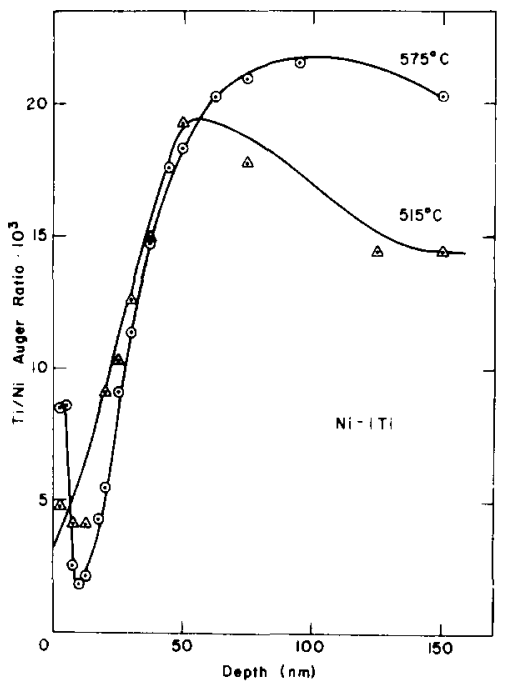

(b)

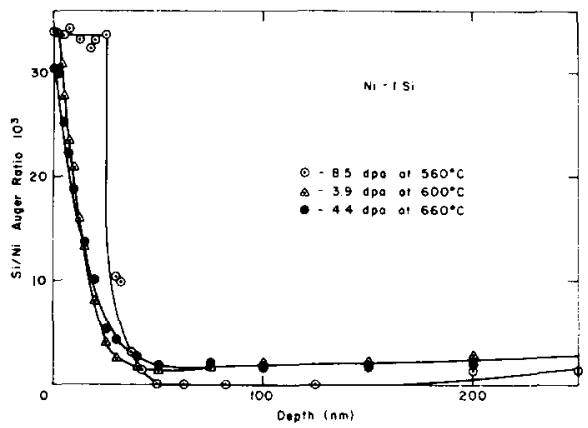

(d)

Fig. 24. Measured concentration vs depth profiles for a (a) $\mathrm{Ni}-1$ at\% $\mathrm{Al}$ alloy irradiated to $10.3 \mathrm{dpa}$ at $510^{\circ} \mathrm{C}$ and $10.7 \mathrm{dpa}$ at $620^{\circ} \mathrm{C}$, (b) $\mathrm{Ni}-1$ at $\% \mathrm{Ti}$ alloy irradiated to $11.2 \mathrm{dpa}$ at $515^{\circ} \mathrm{C}$ and $8.5 \mathrm{dpa}$ at $575^{\circ} \mathrm{C}$, (c) $\mathrm{Ni}-1$ at $\%$ Mo alloy irradiated to $11.6 \mathrm{dpa}$ at $530^{\circ} \mathrm{C}$ and $11.2 \mathrm{dpa}$ at $615^{\circ} \mathrm{C}$, and (d) $\mathrm{Ni}-1$ at\% $\mathrm{Si}$ alloy irradiated to $8.5 \mathrm{dpa}$ at $560^{\circ} \mathrm{C}, 3.9 \mathrm{dpa}$ at $600^{\circ} \mathrm{C}$ and $4.4 \mathrm{dpa}$ at $660^{\circ} \mathrm{C}$. (ref. 51) 
Table 1. Volume misfit parameters based on atomic size or mean atomic volume determined for a number of solute/solvent systems. Note that the discrepancies in the direction of segregation (under RIS) predicted from the King volume misfit parameter [67] are removed when the atomic size volume misfit parameter is used. (from ref. 66)

\begin{tabular}{|c|c|c|c|c|c|c|c|}
\hline $\mathrm{r}_{\mathrm{solv}}(\bar{\AA})$ & Structure & Alloy & $\mathrm{r}_{\mathrm{sol}}(\bar{A})$ & $\begin{array}{c}\text { Structure } \\
\text { of the solute } \\
\text { when } \\
\text { crystalline }\end{array}$ & $\begin{array}{c}\text { Volume } \\
\text { misfit } \% \\
\left(r_{\text {sol }} / r_{\text {solv }}\right)^{3}-1\end{array}$ & $\begin{array}{c}\text { Direction } \\
\text { of } \\
\text { segregation } \\
\text { [62] }\end{array}$ & $\begin{array}{l}\text { Volume } \\
\text { misfit } \% \\
\text { after King } \\
\text { [67] }\end{array}$ \\
\hline 1.3775 & fcc & $\begin{array}{l}\mathrm{Pd}-\mathrm{Cu} \\
\mathrm{Pd}-\mathrm{Fe} \\
\mathrm{Pd}-\mathrm{Mo} \\
\mathrm{Pd} \cdot \mathrm{Ni} \\
\mathrm{Pd}-\mathrm{W}\end{array}$ & $\begin{array}{l}1.278 \\
1.24115 \\
1.36255 \\
1.2458 \\
1.37095\end{array}$ & $\begin{array}{l}f c c \\
b c c \\
b c c \\
f c c \\
b c c\end{array}$ & $\begin{array}{r}-20 \\
-27 \\
-3 \\
-26 \\
-2\end{array}$ & $\begin{array}{l}+ \\
+ \\
+ \\
+ \\
+\end{array}$ & $\begin{array}{r}-19 \\
-12 \\
-4 \\
-14 \\
-4\end{array}$ \\
\hline 1.4315 & fcc & $\begin{array}{l}\mathrm{Al}-\mathrm{Ge} \\
\mathrm{Al}-\mathrm{Si} \\
\mathrm{Al}-\mathrm{Zn}\end{array}$ & $\begin{array}{l}1.2249 \\
1.17585 \\
1.3347\end{array}$ & $\begin{array}{l}\text { diamond } \\
\text { diamond } \\
h \in p\end{array}$ & $\begin{array}{l}-37 \\
-45 \\
-19\end{array}$ & $\begin{array}{l}+ \\
+ \\
+\end{array}$ & $\begin{array}{r}+13 \\
-16 \\
-6\end{array}$ \\
\hline 1.278 & fcc & $\begin{array}{l}\mathrm{Cu}-\mathrm{Ag} \\
\mathrm{Cu}-\mathrm{Be} \\
\mathrm{Cu}-\mathrm{Fe} \\
\mathrm{Cu}-\mathrm{Ni}\end{array}$ & $\begin{array}{l}1.4447 \\
1.1130 \\
2.24115 \\
1.2458\end{array}$ & $\begin{array}{l}\text { fcc } \\
\text { hcp } \\
\text { bcc } \\
\text { fcc }\end{array}$ & $\begin{array}{r}+44 \\
-34 \\
-8 \\
-7\end{array}$ & $\begin{array}{l}- \\
+ \\
+ \\
+\end{array}$ & $\begin{array}{r}+44 \\
-26 \\
+5 \\
-8\end{array}$ \\
\hline 1.2458 & fcc & $\begin{array}{l}\mathrm{Ni}-\mathrm{Al} \\
\mathrm{Ni}-\mathrm{Au} \\
\mathrm{Ni}-\mathrm{Be} \\
\mathrm{Ni}-\mathrm{Cr} \\
\mathrm{Ni}-\mathrm{Ge} \\
\mathrm{Ni}-\mathrm{Mn} \\
\mathrm{Ni}-\mathrm{Mo} \\
\mathrm{Ni}-\mathrm{Si} \\
\mathrm{Ni}-\mathrm{Si} \\
\mathrm{Ni}-\mathrm{Ti}\end{array}$ & $\begin{array}{l}1.4315 \\
1.44205 \\
1.1130 \\
1.2490 \\
1.2249 \\
1.36555 \\
1.36255 \\
1.450 \\
1.17585 \\
1.4478\end{array}$ & $\begin{array}{l}\text { fcc } \\
\text { fcc } \\
\text { hcp } \\
\text { bcc } \\
\text { diamond } \\
\text { fcc ( } \gamma \text {-phase) } \\
\text { bcc } \\
\text { rhomboh. } \\
\text { diamond } \\
\text { hcp }\end{array}$ & $\begin{array}{r}+52 \\
+55 \\
-29 \\
+1 \\
-5 \\
+32 \\
+31 \\
+58 \\
-16 \\
+57\end{array}$ & $\begin{array}{l}- \\
- \\
+ \\
- \\
+ \\
- \\
- \\
+ \\
-\end{array}$ & $\begin{array}{r}+15 \\
+64 \\
<0 \\
+10 \\
+15 \\
+23 \\
+22 \\
+21 \\
-6 \\
+29\end{array}$ \\
\hline 1.4478 & hcp & $\begin{array}{l}\text { Ti-Al } \\
\text { Ti-V }\end{array}$ & $\begin{array}{l}1.4315 \\
1.3112\end{array}$ & $\begin{array}{l}f c c \\
f c c\end{array}$ & $\begin{array}{r}-3 \\
-26\end{array}$ & $\begin{array}{l}+ \\
+\end{array}$ & $\begin{array}{l}-20 \\
-15\end{array}$ \\
\hline 1.24115 & bcc & $\mathrm{Fe}-\mathrm{Cr}$ & 1.2490 & bcc & +2 & - & +4 \\
\hline 1.59855 & $h c p$ & $\mathrm{Mg}-\mathrm{Cd}$ & 1.4894 & hc p & -19 & + & -21 \\
\hline
\end{tabular}


(iii) Dose dependence. The dose dependence of RIS has been measured by Averback et al. [63] in a $\mathrm{Ni}-12.7$ at \% $\mathrm{Si}$ alloy bombarded with $2 \mathrm{MeV} \mathrm{He}^{+}$ions. Since the external surface serves as a sink, the preferential transport of silicon by the defect fluxes causes the silicon concentration at the surface to exceed the solubility limit of $\sim 10$ at\% and a coating of $\mathrm{Ni}_{3} \mathrm{Si}\left(\gamma^{\prime}\right)$ forms. The thickness of the layer grows with increasing dose and the slope of the growth rate curve is the growth rate constant. The data shown in Fig. 25 for irradiation with $2 \mathrm{MeV} \mathrm{\textrm {Li } ^ { 7 }}$ at $520^{\circ} \mathrm{C}$ give a coating growth that is parabolic with time. That is, at a constant dose rate, the coating thickness is proportional to the square root of the dose. The dose dependence is also shown more qualitatively [64] in Fig. 26. The dose dependence of RIS to the surface has also been investigated in three other alloy systems; $\mathrm{Cu}-\mathrm{Ni}, \mathrm{Ni}-\mathrm{Ge}$ and $\mathrm{Ni}-\mathrm{Sb}$. In all three cases, growth of the segregated surface layer was found to be approximately parabolic with dose [72].

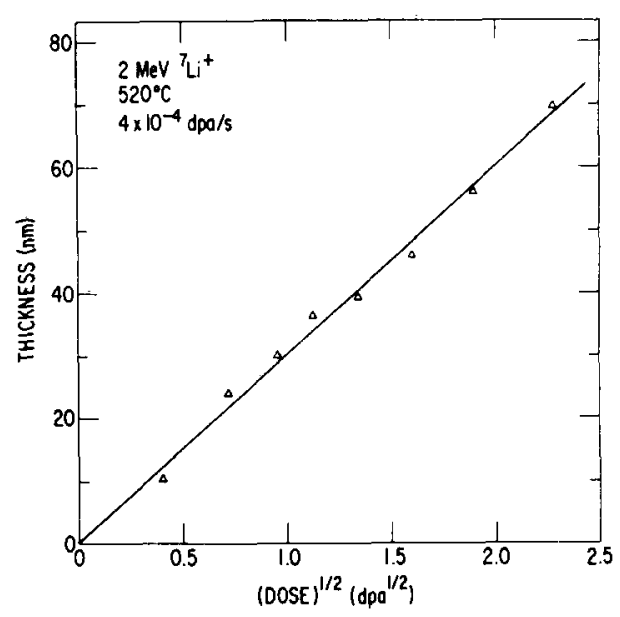

Fig. 25. Thickness of the $\gamma^{\prime}$ surface layer as a function of the square root of dose for $2.0 \mathrm{MeV} \mathrm{Li}$ irradiation at $520^{\circ} \mathrm{C}$ and a dose rate, $K_{0}$ of $4 \times 10^{-4} \mathrm{dpa} / \mathrm{s}$. (from ref. 63)

(iv) Temperature dependence. The temperature dependence of radiationinduced segregation results from the interplay of several factors. At low temperatures at which the irradiation-produced vacancies are relatively immobile, the recombination rate of vacancies and interstitials will be high. This greatly reduces the fraction of defects that annihilates at sinks and consequently the degree of radiation-induced segregation. At high temperatures, enhanced recombination that results from the larger equilibrium vacancy concentration, 
faster back diffusion and the decreased effectiveness of defect-solute binding combine to again inhibit segregation. However, at intermediate temperatures (typically 0.3 to $0.5 \mathrm{~T}_{\mathrm{m}}$ ), significant solute participation in the defect fluxes may occur, resulting in pronounced solute segregation over large distances (10s to $100 \mathrm{~s}$ of nanometers). The temperature dependence of radiation-induced segregation is therefore expected to closely resemble the temperature dependence of the radiation-enhanced portion of the diffusion coefficient.

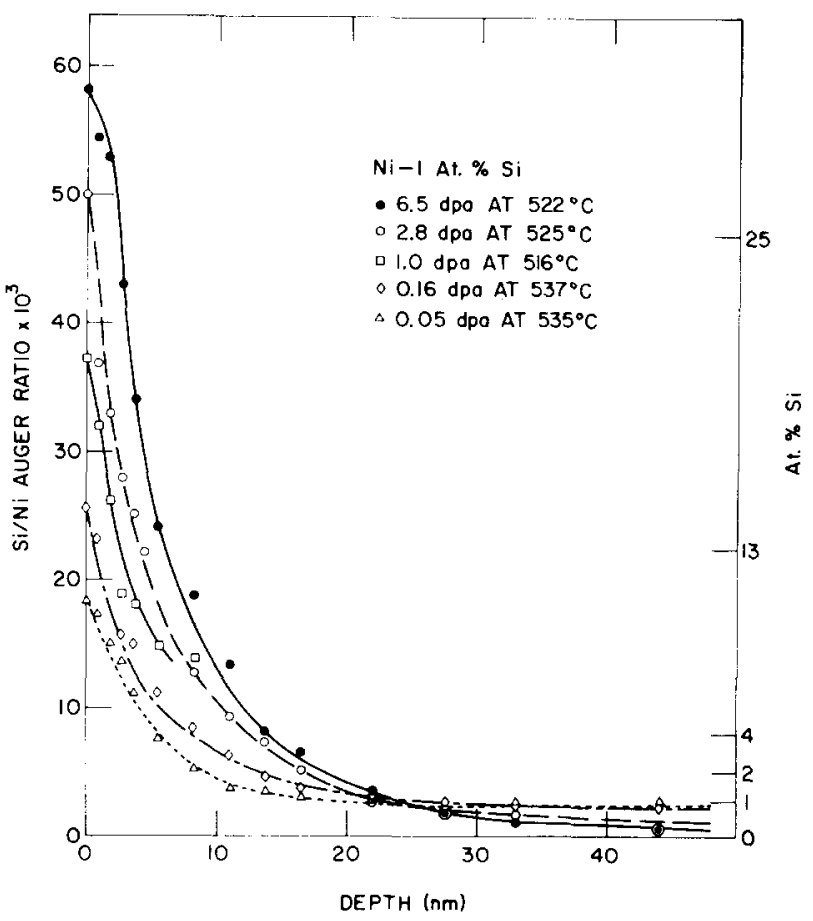

Fig. 26. Si/Ni peak-to-peak ratios as a function of depth from the irradiated surface for a series of $\mathrm{Ni}-1$ at\% $\mathrm{Si}$ alloys irradiated to different doses at a minimal temperature of $525^{\circ} \mathrm{C}$. The right ordinate gives $\mathrm{Si} / \mathrm{Ni}$ peak-to-peak ratios obtained from unirradiated $\mathrm{Ni}-\mathrm{Si}$ alloys of known $\mathrm{Si}$ concentration. (from ref. 64)

The temperature dependence of RIS has been studied by many investigators. Rehn et al. [51] studied the dependence of $\mathrm{Si}$ segregation on irradiation temperature in a $\mathrm{Ni}-1$ at\% $\mathrm{Si}$ alloy bombarded with $3.5 \mathrm{MeV} \mathrm{Ni}^{+}$ions, and Averback et al. [63] studied the same dependence in a $\mathrm{Ni}-12.7$ at\% $\mathrm{Si}$ alloy during $2 \mathrm{MeV} \mathrm{He}^{+}$bombardment. Since the external surface curves as a sink, the preferential transport of silicon by the defect fluxes causes the silicon concentration at the surface to exceed the solubility limit of $\sim 10$ at $\%$ and a 
coating of $\mathrm{Ni}_{3} \mathrm{Si}\left(\gamma^{\prime}\right)$ forms. The thickness of the layer grows with increasing dose and the slope of the growth rate curve is the growth rate constant. When plotted as a function of temperature, Fig. 27, the peak in the growth rate occurs at an intermediate temperature as expected from theory. At low temperature the growth rate is low due to the high recombination rate of vacancies and interstitials resulting from the high excess vacancy concentration due to the low vacancy mobility. At high temperature the growth rate also diminishes due to the increase in the equilibrium vacancy concentration and thus, recombination. But at intermediate temperatures, the segregation peaks. The same is true for irradiation of Ni-1at\% Si, Fig. 28. Several others have investigated the temperature behavior of RIS and found the same qualitative dependence [51,55].

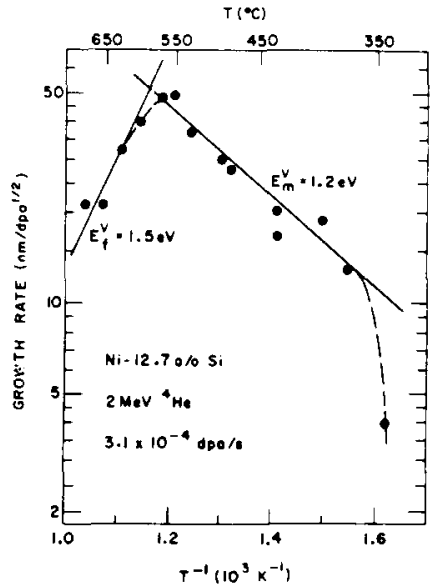

Fig. 27. Arrhenius plot of the measured growth rates of $\gamma^{\prime}$-coatings on $\mathrm{Ni}-12.7$ at\% $\mathrm{Si}$ specimens during 2 $\mathrm{MeV} \mathrm{He}^{4}$ bombardment at a dose rate of $3.1 \times 10^{-4} \mathrm{dpa} / \mathrm{s}\left(4.7 \times 10^{14}\right.$ ions $/ \mathrm{cm}^{2} \cdot \mathrm{s}$ ). (from ref. 62 )

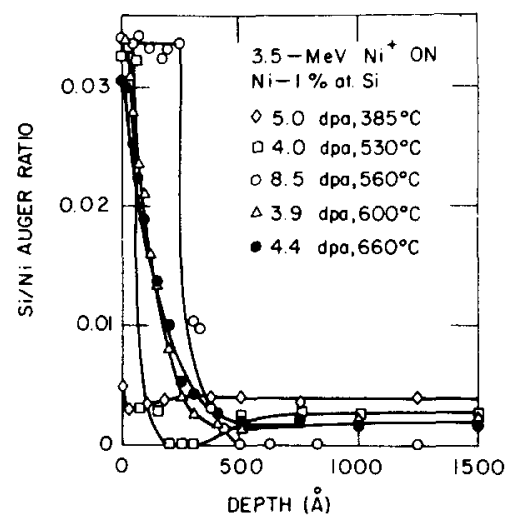

Fig. 28. Si/Ni peak-to-peak ratios versus depth from the irradiated surface for a series of $\mathrm{Ni}-1$ at\% $\mathrm{Si}$ alloys irradiated at various temperatures and doses. $\mathrm{A} \mathrm{Si} / \mathrm{Ni}$ ratio of 0.033 corresponds to 25 at\% Si. (from ref. 51)

(v) Dose rate effect. The magnitudes of the defect fluxes, which determine the degree of radiation-induced segregation near sinks, are temperature and dose rate dependent. Since the minimum in the defect recombination rate shifts toward lower temperatures for lower damage rates, decreasing the defect production rate is expected to decrease the temperature where maximum segregation occurs. Current theory also predicts that the RIS growth rate constant should vary inversely as the fourth root of the dose-rate in the recomibination-limited temperature regime [62]. Averback et al. [63] also 
investigated the dose rate effect on the radiation-induced formation of $\mathrm{Ni}_{3} \mathrm{Si}$, Fig. 29. The predicted fourth-root dependence in the recombination-limited regime is indicated by the dotted line. The observed dependence of the RIS rate on dose-rate is slightly weaker than predicted, but the agreement is considered reasonable. Since the vacancy concentration during irradiation at high temperatures is approximately equal to the equilibrium vacancy concentration, the amount of defect annihilation per unit dose is independent of dose-rate, and and no effect of the dose-rate on the growth rate constant is predicted at high temperatures. As can be seen from Fig. 29, the experimental results support this conclusion in the case of $\mathrm{Ni}-12.7$ at\% $\mathrm{Si}$.

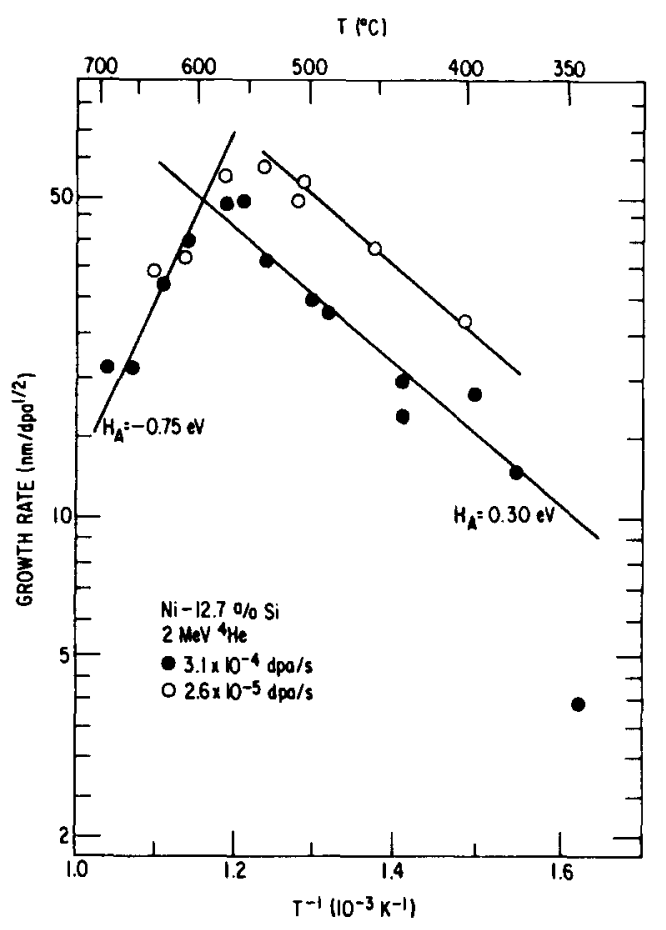

Fig. 29. Arrhenius plot of the growth-rate constant for two dose rates $3.1 \times 10^{-4}$ $\mathrm{dpa} / \mathrm{s}$ (closed symbols) and $2.6 \times 10^{-5} \mathrm{dpa} / \mathrm{s}$ (open symbols). (from ref. 63)

The dependence of RIS on dose rate in $\mathrm{Ni}-10$ at\% $\mathrm{Ge}$ has also been studied [77]. RBS measurements clearly show that during irradiation with $2 \mathrm{MeV} \mathrm{He}^{+}$at $450^{\circ} \mathrm{C}$, more $\mathrm{Ge}$ is transported to the surface at a dose rate of $2.6 \times 10^{-5} \mathrm{dpa} / \mathrm{s}$ than at $3.2 \times 10^{-4} \mathrm{dpa} / \mathrm{s}$ over a range (0.04-1.0 dpa) of total doses. These results are in qualitative agreement with those for Ni-Si. 
(vi) Homogeneous alloys. Some very striking examples of solute redistribution by RIS have been observed by Robrock and Okamoto and reported by Rehn and Wiedersich [72]. In the case of a Ni-6 at\% $\mathrm{Si}$, single phase, solid solution alloy irradiated with $\mathrm{MeV}$ ions, the defect generation rate does not vary strongly with depth over the first few hundred nanometers. At these shallow depths of penetration, the surface serves as the dominant sink. Silicon is preferentially transported to the surface until the solubility limit $(10$ at $\%)$ is exceeded, where upon $\mathrm{Ni}_{3} \mathrm{Si}\left(\gamma^{\prime}\right)$ begins to precipitate. As silicon is transported to the surface from the interior, the layer thickness grows until the effectiveness of the sink diminishes relative to internal sinks. Then, precipitation begins to occur at these sinks with the same result. Voids, dislocation loops and grain boundaries all become coated with $\gamma^{\prime}$. Hence, RIS has decomposed a single phase alloy into a two-phase material.

A similar spatial redistribution occurs when the solute atoms are preferentially transported away from sinks. The result is an enrichment in solute a distance away from the sink. If the matrix composition is close to the solubility limit, precipitation may occur in these regions (since they are usually rather large compared to the solute depleted regions and only small changes in composition are expected) and the solid solution alloy is decomposed into a twophase structure.

RIS in two-phase alloys can redistribute the phases by solute redistribution. Thermally aged $\mathrm{Ni}-\mathrm{Al}$ alloys result in a uniform distribution of cuboidal $\mathrm{Ni}_{3} \mathrm{Al}$ $\left(\gamma^{\prime}\right)$ particles. During irradiation, aluminum depletion shifts the local composition around sinks into the solid solution range [78], Precipitate-denuded zones form at the surface and around internal sinks such as dislocation loops, and $\gamma^{\prime}$ becomes concentrated in the sink-free areas.

(vii) Damage distribution and bombarding ion effects. When the rate of defect production during irradiation varies with ion depth, radiation-induced segregation, which is driven by gradients in the point-defect concentration, $C_{d}$, can become significant. Early in the irradiation, the composition of the alloy is still fairly uniform and the flux, $J_{A}$, of element $A$ is essentially proportional to $\nabla$ $C_{d}$. From the diffusion equation,

$$
\partial \mathrm{C}_{\mathrm{A}} / \partial \mathrm{t}=-\nabla \mathrm{J}_{\mathrm{A}},
$$

it follows that the divergence of the defect flux, $\nabla^{2} C_{d}$, is therefore a measure of the rate of accumulation of solute at a given depth. Examples of the variation in 
$K_{0}, C_{d}, d C_{d} / d x$, and $d^{2} C_{d} / d x^{2}$ with distance from the free surface are shown in Figs. 30 and 31 for low- and high-energy ions, respectively [72]. Note that positive values of $d^{2} C_{d} / d x^{2}$ will cause solute accumulation if the solute and defect fluxes move in the same direction, but solute depletion if the fluxes are in opposite directions. By this reasoning it can be seen that these examples are representative of solute redistribution via the interstitial inverse Kirkendall effect. In these cases, solute enrichment will occur in regions along the ion range where the defect profile is concave upward, while regions that are concave downward will be depleted of solute. In both the low- and high-energy cases, solute enrichment occurs at the bombarded surface and just beyond the damage peak, while the peak and near surface regions are depleted of solute. A difference between the high- and low-energy ion bombardment occurs in the midrange region, about halfway between the damage peak and the surface, where solute enrichment occurs for high-energy ions but not for low energy ions.

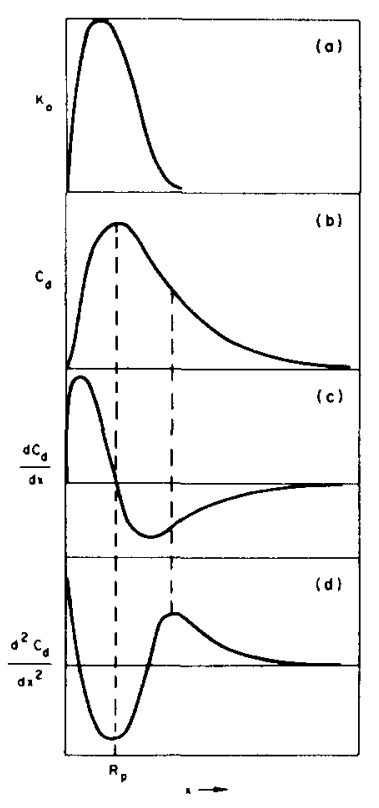

Fig. 30. Schematic plots of (a) defect production rate $\mathrm{K}_{\mathbf{0}}$, (b) steady-state defect concentration $C_{d}$, (c) $d C_{d} / d x$, and (d) $d^{2} C_{d} / d x^{2}$ versus depth for low-energy ions. (from ref. 72)

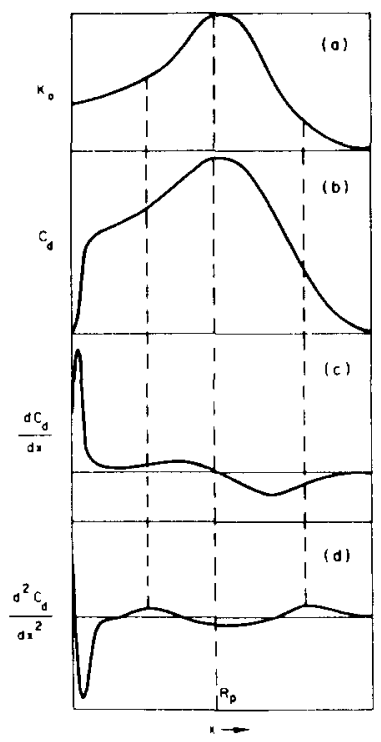

Fig. 31. Schematic plots of (a) defect production rate $K_{0}$, (b) steady-state defect concentration $C_{d}$, (c) $d C_{d} / d x$, and (d) $d^{2} C_{d} / d x^{2}$ versus depth for high-energy ions. (from ref. 72) 
In addition to differences in solute redistribution due to ion energies, the mass of the ion can significantly affect the degree of radiation induced segregation [72]. This is due to the variation in the efficiency for producing freely migrating defects (which fuels RIS) with ion type. Figure 32 shows the relative efficiencies (normalized to that of $1 \mathrm{MeV}$ protons) for defect production as a function of the defect-production weighted-average recoil energy, $P_{1 / 2} . P_{1 / 2}$ is the primary recoil energy above and below which half of the defects are produced. It gives a measure of the spatial distribution of the defect production; the larger the value of $P_{1 / 2}$, the greater is the tendency for defects to be produced in cascades rather than as isolated defects. This figure provides a quantitative representation of the relative efficiency for producing solute redistribution at elevated temperatures as a function of the hardness of the primary recoil spectrum.

Qualitatively, as recoil events increase in energy from tens to hundreds of electron volts, Frenkel pair production changes from the introduction of randomly distributed isolated Frenkel defects to the generation of defect pairs in close proximity. Cascade regions form for primary recoil energies above $1 \mathrm{keV}$. Hence, since RIS is tied to the efficiency of defect production, the degree of the observed segregation can be expected to vary substantially depending upon the ion and the energy used during the irradiation.



Fig. 32. Relative efficiencies of various ions for producing long-range migrating defects at elevated temperature plotted as a function of the weighted-average recoil energy. (from ref. 72)

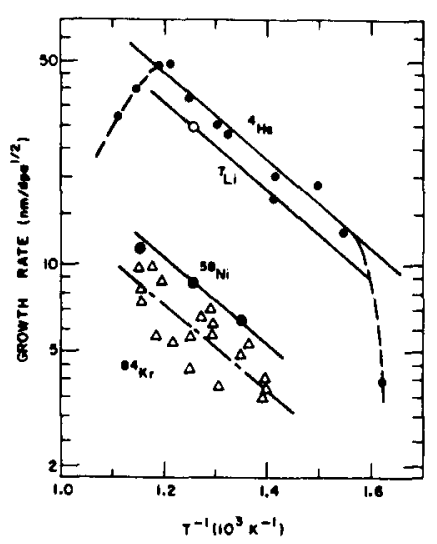

Fig. 33. Arrhenius plot of the growth rates of $\gamma^{\prime}$ coatings on $\mathrm{Ni}-12.7$ at\% $\mathrm{Si}$ specimens for different ions. The calculated displacement rates were kept approximately constant at $3 \mathrm{x}$ $10^{-4} \mathrm{dpa} / \mathrm{s}$. (from ref. 62 ) 
A large reduction in the growth rate of $\gamma^{\prime}$ coatings on $\mathrm{Ni}-12.7$ at $\% \mathrm{Si}$ alloy, with ion mass was observed by Rehn [62] while keeping the near-surface displacement rate constant for all ion masses, Fig. 33. In this case, it was found that sinks produced by the heavier ion bombardment significantly reduced RIS to the surface at $485^{\circ} \mathrm{C}$. However, experimental evidence also shows that defect losses within the denser cascades also contributed to the reduction in RIS.

\section{F. Gibbsian adsorption}

The readjustment of the surface composition of a homogeneous alloy to a composition different than that in the bulk, in an effort to minimize the free energy of the system, is known as Gibbsian adsorption (GA) [79]. Also known as thermal surface segregation, this process can lead to substantial changes in composition in the first one or two atom layers at the surface, while leaving the bulk composition practically unaffected due to the large bulk-to-surface-volume ratio. The readjustment occurs spontaneously at temperatures sufficiently high for diffusion to proceed at reasonable speed. At equilibrium, the atom fractions of $A$ and $B$ in the "surface phase", $C_{A} s$ and $C_{B} s$, are related to the respective atom fractions in the "bulk phase", $C_{A}$ b and $C_{B}$ b, by the relationship [79]

$$
C_{A} s / C_{B} s=\left(C_{A}^{b} / C_{B}^{b}\right) \exp \left(-\Delta G_{a} / k T\right),
$$

where $\Delta \mathrm{G}_{\mathrm{a}}=\Delta \mathrm{H}_{\mathrm{a}}-\mathrm{T} \Delta \mathrm{S}_{\mathrm{a}}$ is the GA free energy, with $\Delta \mathrm{S}_{\mathrm{a}}$ and $\Delta \mathrm{H}_{\mathrm{a}}$ being the entropy and enthalpy of adsorption for the A component, respectively.

During the concentration buildup towards equilibrium, the net flux of $A$ atoms into the surface atomic plane, $\mathrm{J}_{\mathrm{A}}$, is calculated by [80]

$$
\Omega J_{A}=\left(v_{A}^{b->s} C_{A}{ }^{b} C_{B} s-v_{A}^{s->b} C_{A} s C_{B} b\right) \xi,
$$

where $\xi$ is the atomic layer thickness, and the surface-to-bulk jump frequency $v_{A} s>b$ is related to the bulk-to-surface jump frequency $v_{A}{ }^{b->s}$ by the following equation based on the condition of equilibrium $\left(\mathrm{J}_{\mathrm{A}}=0\right)$

$$
v_{A}^{s->b}=v_{A}{ }^{b->s} \exp \left(\Delta G_{a} / k T\right) .
$$


Since $v_{A} b->s$ is a function of defect jump frequencies by exchange with $A$ atoms and defect concentrations in the bulk, then

$$
v_{A}^{b->s}=v_{A v} C_{v}^{b}+v_{A i} C_{i}^{b}
$$

and GA can be strongly enhanced by irradiation at temperatures below $\sim 0.6 \mathrm{~T}_{\mathrm{m}}$.

The dependence of Gibbsian adsorption on temperature has recently been measured on a number of alloys [81-83] using ion scattering spectroscopy, and the $1 / \mathrm{T}$ dependence of $\ln \left[\mathrm{C}_{\mathrm{A}} \mathrm{s} / \mathrm{C}_{\mathrm{B}} \mathrm{s}\right]$, from eqn. (3.63), has been confirmed for Ni$\mathrm{Cu}$ and $\mathrm{Ni}-\mathrm{Au}$ alloys $[81,82]$.

Gibbsian adsorption involves compositional changes in the first one or two atom layers, which is a comparable depth scale to the effects of preferential sputtering. Hence, under ion bombardment, the surface composition will be affected by both of these processes. Lam and Wiedersich [80] provided a schematic description of the dynamic behavior of the surface composition during ion bombardment resulting from the simultaneous effects of GA and PS. In this example, Fig. 34, Gibbsian adsorption results in a surface concentration of A atoms that is initially greater than the bulk level. This leads to enhanced preferential sputtering of $A$ atoms since the sputtered atom flux is primarily from the first atom layer. Consequently, the concentration of $A$ atoms in the subsurface layer will be significantly reduced in an effort to re-establish thermodynamic equilibrium. However, at steady state, the composition of the sputtered atom flux is equal to the bulk composition of the alloy.

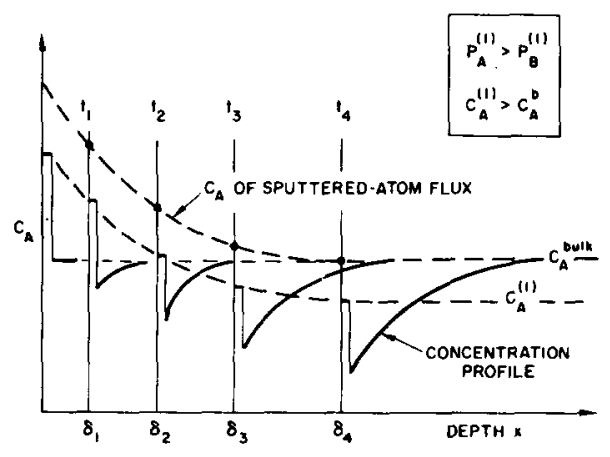

Fig. 34. Schematic description of the simultaneous effects of GA and PS on the time evolution of the sputtered-atom flux composition and alloy composition in the near surface region. (from ref. 80) 


\section{G. Sputtering}

(i) Basic model. Sputtering is a key element in determining surface composition under ion bombardment, primarily through the action of preferential sputtering. However, all of the processes discussed thus far will contribute to the observed effects. Since different elements sputter with different probabilities, and the surface composition is a function of this probability as well as the processes of ion implantation, displacement mixing, radiation-enhanced diffusion, radiationinduced segregation and Gibbisan adsorption, the resulting surface composition will also depend on these processes. This section will briefly describe the process of physical sputtering, followed by a discussion of preferential sputtering as it affects surface compositional changes in alloys.

Sigmund [84-86] developed the basic theory and description for the sputter yield due to linear cascade sputtering, and Winters [87] provided a clear and concise summary of theory and experiment. Linear cascade sputtering refers to conditions on the collision cascade. A collision cascade is linear if only a minor fraction of the target atoms within the cascade volume is set in motion. For a bulk cascade, this implies a low density of point defects generated. As applied to sputtering, it means that the sputter yield must be small compared to the number of target atoms located within the surface area affected by a bombarding particle. In practice, cascades in metals are close to linear except those generated by rather heavy ions bombarding heavy targets in the energy range from $\sim 10 \mathrm{keV}$ to $\sim 1 \mathrm{MeV}$. The linear sputter yield formula is

$$
\mathrm{Y}=\Lambda \mathrm{F}_{\mathrm{D}} \text {, }
$$

where $\mathrm{Y}$ is the number of atoms emitted per incident particle, $\mathrm{L}$ contains all the material properties and incident ion angular dependence and $F_{D}$ is the density of deposited energy per unit depth at the surface and depends on the type, energy and direction of the incident ion and the target parameters $Z_{2}, M_{2}$ and $N$. The derivation of $\Lambda$ involves a description of the number of recoil atoms that can overcome the surface barrier and escape from the solid. Sigmund [84] has derived an expression for $\Lambda$ using the Thomas-Fermi screening function

$$
\Lambda=0.042 / \mathrm{NU}_{\mathrm{o}}
$$

where $\mathrm{N}$ is the atomic number density and $U_{0}$ is the surface binding energy and can be estimated from the heat of sublimation. The deposited energy $F_{D}$ is given as 


$$
F_{D}=\alpha N S_{n}(E)
$$

where $\alpha$ is a dimensionless quantity depending on the relative masses and angle of incidence [84], and $S_{n}(E)$ is the nuclear stopping power. For keV energies and heavy-to-medium mass ions, the expression for the nuclear stopping power is calculated by Lindhard [10,88], using a Thomas-Fermi cross section [89],

$$
S_{n}(E)=4 \pi Z_{1} Z_{2} e^{2} a\left[M_{1} /\left(M_{1}+M_{2}\right)\right] S_{n}(\varepsilon),
$$

where $S_{n}(\varepsilon)$ is the reduced stopping power, a is the screening radius, $Z_{1}, M_{1}$ and $Z_{2}, M_{2}$ are the atomic numbers and masses of incident ion and target atom, respectively, and

$$
\varepsilon=a M_{2} E /\left[Z_{1} Z_{2} e^{2}\left(M_{1}+M_{2}\right)\right] .
$$

The sputtering yield is therefore

$$
\mathrm{Y}=0.528 \alpha \mathrm{Z}_{1} \mathrm{Z}_{2} \mathrm{M}_{1} /\left[\mathrm{M}_{1}+\mathrm{M}_{2}\right] \mathrm{S}_{\mathrm{n}}(\varepsilon) / \mathrm{U}_{\mathrm{o}} .
$$

This model has undergone extensive experimental testing and has enjoyed considerable success. However, systematic deviations have been pointed out for some cases such as light-ion sputtering and low-energy sputtering. Current theories are summarized by Mashkova and Molchanov [90]. Yamamura et al. [91] have proposed a new empirical formulation of the sputtering yield to account for both light-ion and low-energy sputtering in the sputtering yield. Kelly, Falcone and Oliva have undertaken a full re-examination of collisional sputtering theory. In this re-analysis, they have addressed the treatment of the collision cross section [92], the surface binding energy [93], the scaling of deposited energy [94], the characteristic depth and cross section for low-energy elastic collisions [95], the effect of crystallinity [96] and preferential sputtering effects [97]. It is not the intention of this paper to discuss basic sputtering theory, but rather the means by which sputtering influences the composition of bombarded surfaces. With these formulations in hand, we can now discuss the effect of sputtering on compositional changes in the target.

(ii) Preferential sputtering. Wiedersich et al. [98], proposed a simple concept and definition of preferential sputtering by writing the yield or number of A- 
atoms of an alloy per incident ion in the flux of sputtered atoms as

$$
\mathrm{Y}_{\mathrm{A}}=\int_{0}^{\infty} \sigma \mathrm{A}(\mathrm{x})\left(\mathrm{c}_{\mathrm{A}}(\mathrm{x}) / \Omega\right) \mathrm{d} \mathrm{x},
$$

where $\sigma_{A}(x)$ is the cross section for $A$-atoms at a depth $x \geq 0$ to be ejected from the surface, $x=0$, into the region $x<0$ per incoming ion, $C_{A}(x)$ is the atomic fraction of $A$ in the alloy at depth $x$, and $\Omega$ is the mean atomic volume. He then goes on to define $\mathrm{p}_{\mathrm{A}}(\mathrm{x}) \equiv \sigma_{\mathrm{A}}(\mathrm{x}) / \Omega$, where $\mathrm{p}_{\mathrm{A}}(\mathrm{x})$ is the probability per unit depth that an A-atom present at depth $x$ is ejected by an incoming ion. The yield of A-atoms then takes the form

$$
Y_{A}=\int_{0}^{\infty} p A(x)\left(C_{A}(x) d x,\right.
$$

In this form, the distinction introduced by Sigmund [99], between the primary and secondary effects in alloy sputtering is made explicit. The primary effects are those related to the individual sputtering events and the physical variables contributing to the sputter yield are all contained in the sputter probability, $\mathrm{p}_{\mathrm{A}}$, which depends on the type and energy of the incoming ion, the type of the sputtered atom and its surface binding energy, etc. Since the values of the sputter probabilities, $\mathrm{p}_{\mathrm{i}}$, will differ for differing atomic species, preferential sputtering will occur.

The secondary effects in alloy sputtering enter into eqn. (3.72) via the atomic concentration, $C_{A}$, which gives the probability that a site is occupied by an Aatom. All processes mentioned earlier that affect the surface composition during bombardment, eg., recoil implantation, Gibbsian adsorption, RIS, etc., but not preferential sputtering, enter primarily through their effects on the near-surface concentration. As a consequence, the sputter yields of the alloying components will be affected through the factor $C_{A}(x)$ in eqn. (3.72).

Practically, since sputtered atoms come from a shallow layer as shown by Sigmund $[84,85]$, and later by Falcone and Sigmund [100] the integral in eqn (3.72) can be replaced by

$$
\mathrm{Y}_{\mathrm{A}} \sim \overline{\mathrm{p}_{\mathrm{A}}} \mathrm{C}_{\mathrm{A}} \mathrm{s},
$$

where $\overline{p_{A}}$ is the average total probability for an $A$-atom present in the surface layer to be sputtered off per incident ion and $C_{A} s$ is the average atomic concentration of $\mathrm{A}$ in the surface layer. The thickness of this layer is not well defined but should be taken as one or two atomic layers for determining $C_{A} s$ 
since the origin of sputtered ions is heavily weighted toward the first atomic layer.

Differences in the sputter probabilities for component atoms in an alloy are caused by differences in the amounts of energy and momentum transferred to atoms of different masses, and surface binding energies. However, continued sputtering of a semi-infinite alloy target of uniform bulk composition must eventually lead to a steady state in which the composition of the flux of sputtered atoms leaving the surface equals the composition of the bulk alloy. Lam and Wiedersich [80] have shown that bombardment of a binary alloy AB with a flux of ions $\phi$ (ions $/ \mathrm{cm}^{2} \mathrm{~s}$ ) leads to an atom removal rate given by

$$
\mathrm{dN} / \mathrm{dt}=\phi\left(\mathrm{Y}_{\mathrm{A}}+\mathrm{Y}_{\mathrm{B}}\right)
$$

Therefore, the rate at which the sputtered surface recedes can be calculated from the total rate of atom loss per unit area,

$$
\dot{\delta}=\mathrm{d} \delta / \mathrm{dt}=\phi \Omega \mathrm{dN} / \mathrm{dt}=\phi \Omega\left(\mathrm{p}_{\mathrm{A}} \mathrm{C}_{\mathrm{A}} \mathrm{s}+\mathrm{p}_{\mathrm{B}} \mathrm{C}_{\mathrm{B}} \mathrm{s}\right)
$$

where $\delta$ is the thickness of the surface layer removed by sputtering.

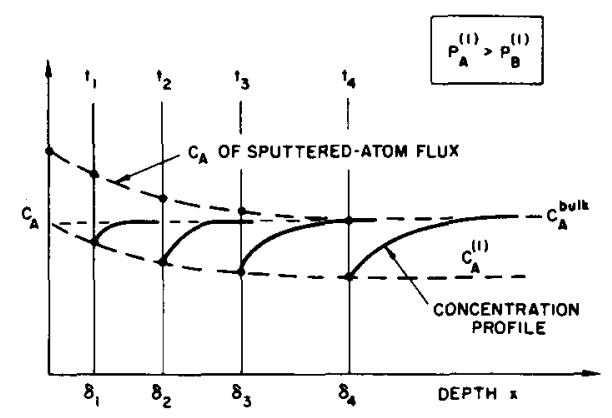

Fig. 35. Schematic description of the preferential sputtering effect on the time evolution of the composition of the sputtered-atom flux and of the near-surface region in the alloy. (from ref. 80)

Lam and Wiedersich [80] have described the time evolution of PS on the nearsurface composition for a binary alloy $A B$ with $\overline{p_{A}} s>\overline{p_{B}} s$, i.e. for the case where PS of A atoms occurs, Fig. 35. Note that initially, the concentration of $A$ atoms in the sputtered flux is larger than in the bulk. However, as the surface 
composition changes and the near-surface layer composition is altered, a steady state will be achieved after a certain bombardment time, when the composition of the sputtered-atom flux becomes equal to the bulk composition, as dictated by the law of conservation of matter. At steady state, the following condition must be fulfilled,

$$
\mathrm{Y}_{1}: \mathrm{Y}_{2}: \mathrm{Y}_{3} \ldots=\mathrm{C}_{1} \mathrm{~b}: \mathrm{C}_{2}^{\mathrm{b}}: \mathrm{C}_{3} \mathrm{~b} \ldots
$$

that is, the ratios of the alloy components in the sputtered flux are the same as those of the bulk alloy and their concentrations are just uniformly diluted by the reemitted sputter ions. Combining this result with eqn. (3.73) the ratio of the total sputter probabilities is

$$
\overline{p_{1}}: \overline{p_{2}}: \ldots=\left(C_{1} b / C_{1} s\right):\left(C_{2} b / C_{2} s\right): \ldots .
$$

i.e., after steady state is attained, the sputter probabilities are proportional to the ratio of the bulk and surface concentrations of the element in question.

\section{H. Phenomenological model for surface composition changes}

Accounting for the effects of all the processes described in the previous sections, Lam \& Wiedersich [80] constructed a phenomenological model for bombardment-induced composition modification by formulating a set of coupled partial differential equations describing the temporal and spatial evaluation of defect and atom concentrations during ion bombardment of a binary alloy. The formulation was based on the set of diffusion and reaction rate equations, i.e., Fick's second law with source and sink terms, describing the time-rate of change of the alloy composition and defect concentrations,

$$
\begin{gathered}
\partial \mathrm{C}_{\mathrm{v}} / \partial \mathrm{t}=-\nabla \cdot\left(\Omega \mathrm{J}_{\mathrm{v}}\right)+\mathrm{K}_{\mathrm{o}}-\mathrm{R}, \\
\partial \mathrm{C}_{\mathrm{v}} / \partial \mathrm{t}=-\nabla \cdot\left(\Omega \mathrm{J}_{\mathrm{i}}\right)+\mathrm{K}_{\mathrm{o}}-\mathrm{R}, \\
\partial \mathrm{C}_{\mathrm{A}} / \partial \mathrm{t}=-\nabla \cdot\left[\left(\Omega \mathrm{J}_{\mathrm{A}}\right)-\mathrm{D}_{\mathrm{A}} \operatorname{disp} \nabla \mathrm{C}_{\mathrm{A}}\right],
\end{gathered}
$$

where $K_{0}$ and $R$ are the local, spatially dependent rates of production and recombination of vacancies and interstitials and $D_{A}$ disp is the diffusion coefficient 
caused solely by the displacement process. The time-dependent atom and defect concentration distributions can be determined by solving eqns (3.78) through (3.80) numerically for a semi-infinite target using appropriate starting and boundary conditions as described by Lam $[80,101,102]$. This formulation covers the processes of DM, RED and RIS. Gibbsian adsorption and preferential sputtering can be accommodated in the model by treating the surface layer as a separate phase. Because of the structure of the phenomenological model, calculations can be made to determine the dependence of surface and subsurface compositional changes on material and irradiation variables as well as isolating the contributions of individual processes. However, because many of the parameters needed in the models are unknown, quantitative comparisons with experiment are difficult. Nevertheless, semiquantitative modeling of bombardment-induced compositional redistribution in several binary alloys have been made.

Lam and Wiedersich [80] calculated the composition distributions in a $\mathrm{Ni}-40$ at\% $\mathrm{Cu}$ alloy bombarded with $3 \mathrm{keV} \mathrm{Ni}{ }^{+}$at $500^{\circ} \mathrm{C}$. Figure 36 shows the spatial distribution of $\mathrm{Cu}$ atoms for a number of sputtering times. The calculated peak damage rate was $3.5 \times 10^{-2} \mathrm{dpa} / \mathrm{s}$ and corresponds to an ion flux of $3.75 \times 10^{13}$ $/ \mathrm{cm}^{2} \mathrm{~s}$. The peak damage occurs at a depth of $\sim 1 \mathrm{~nm}$. The projected range is $\sim 7$ $\mathrm{nm}$ and the spatially dependent damage rate is shown by the dashed curve in the to graph for time $t=0$. The thickness of the surface layer removed by sputtering is indicated for various times. Since the initial starting conditions correspond to the thermodynamic equilibrium state of the alloy, with an equilibrated $\mathrm{Cu}$ enrichment due to $\mathrm{GA}$ at the surface, the $\mathrm{Cu}$ surface concentration, $\mathrm{C}_{\mathrm{Cu}}$ s is very high initially, then decreases with sputtering time, and finally attains a steady state value after $\sim 10^{4} \mathrm{~s}$.

The temperature dependence of the bombardment-induced compositional changes as measured by the time evolution of $\mathrm{C}_{\mathrm{Cu}}$ s and the steady state concentration profiles is shown in Fig. 37. At short sputtering times, $t \leq 10 \mathrm{~s}$, GA controls the magnitude of $\mathrm{C}_{\mathrm{Cu}}$ s, when starting from the equilibrium state, at which $\mathrm{C}_{\mathrm{Cu}} \mathrm{s}$ is nearly 100 at\%, Fig 37a. With increasing time, however, PS and RIS lead to a gradual decrease in $\mathrm{C}_{\mathrm{Cu}}$ s towards a steady state value. At steady state, the surface alloy composition, $\mathrm{C}_{\mathrm{Cu}} \mathrm{s} / \mathrm{C}_{\mathrm{Ni}} \mathrm{s}$, is determined by the relative contributions of the first and second layers to the sputtered atom flux and the bulk composition, in such a way that the compositions of the sputtered atom flux and the bulk alloy become equal. 


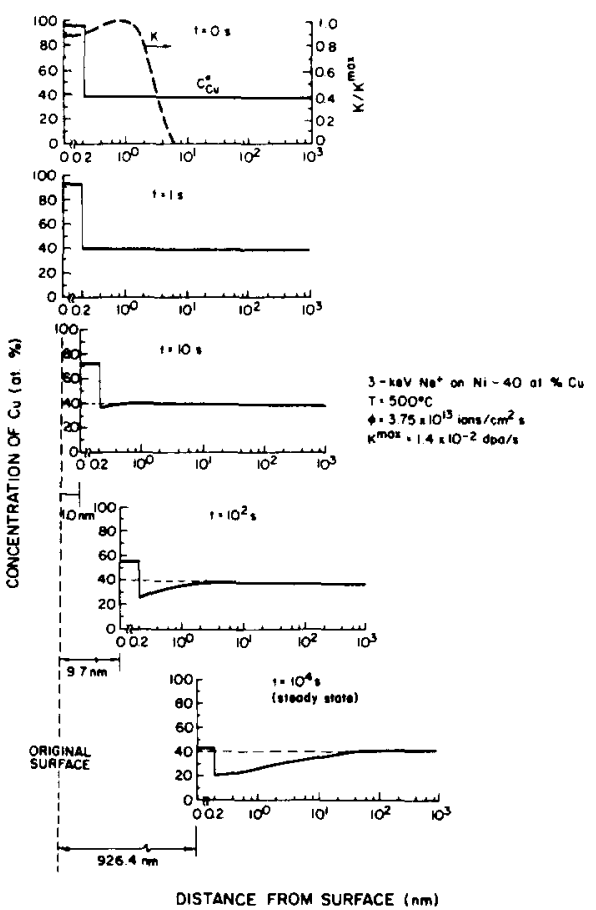

Fig. 36. Calculated time evolution of $\mathrm{Cu}$ concentration profiles in a $\mathrm{Ni}-40$ at $\% \mathrm{Cu}$ alloy sputtered with $3-\mathrm{keV} \mathrm{Ne} \mathrm{Ne}^{+}$ions at $500^{\circ} \mathrm{C}$. The profile of the damage rate $\mathrm{K}_{\mathrm{o}}$ is shown by the dashed curve in the top portion, and the thickness of the surface layer removed by sputtering is indicated. The energies for vacancy migration via $\mathrm{Cu}$ and $\mathrm{Ni}$ atoms were taken to be 0.95 and $0.97 \mathrm{eV}$, respectively. (from ref. 80 )

Figure 37b shows the steady state concentration profiles in the subsurface region calculated for various temperatures. Near room temperature, where point-defect mobility is limited, PS and DM are the main processes that govern the development of the alloy composition in the altered layer, which extends to a depth approximately equal to the damage range. The additional effect of GA, which is quite small at this temperature, is reflected by the noticeable difference between $\mathrm{C}_{\mathrm{Cu}} \mathrm{s}$ in layers 1 and 2 . Above $\sim 100^{\circ} \mathrm{C}, \mathrm{GA}, \mathrm{RED}$ and RIS become significant, and effectively determine the extent of and the composition in the altered layer. The higher the temperature, the thicker the altered layer. At $700^{\circ} \mathrm{C}$, the thickness of this layer is $\sim 4 \mu \mathrm{m}$, which is $\sim 600$ times larger than the damage range. Compositional changes at such large depths suggest that the effects of RED and RIS are profound at elevated temperatures. The long times which are necessary to achieve steady state at high temperatures attest to the extension of the concentration gradient to such depths. 

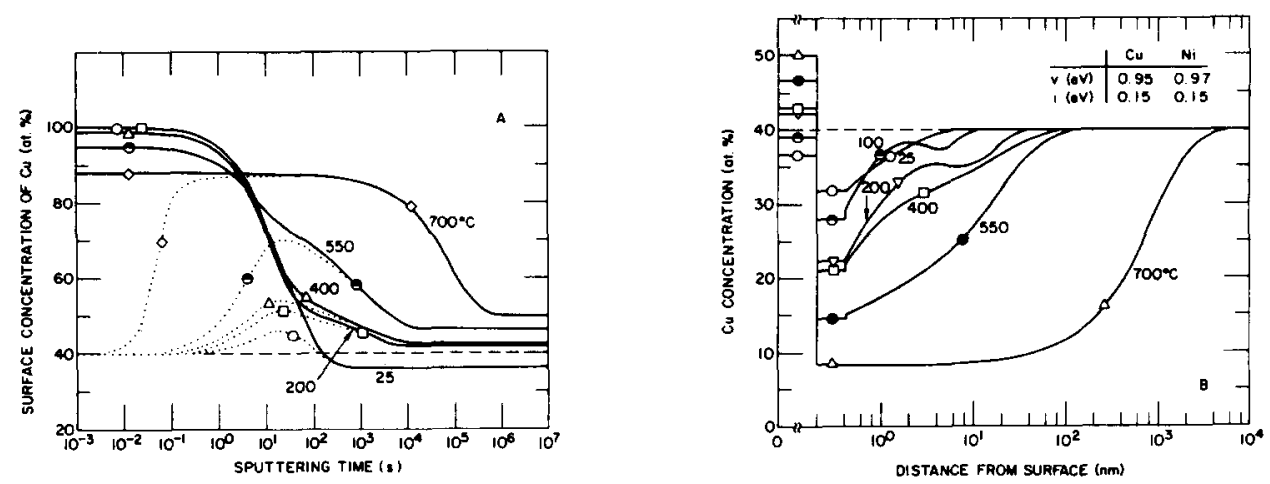

Fig. 37. (a) Calculated time evolution of the composition in the outermost atom layer of a $\mathrm{Ni}-40$ at $\% \mathrm{Cu}$ alloy during $3-\mathrm{keV} \mathrm{Ni}^{+}$sputtering at various temperatures. Two sets of initial conditions were used: the thermodynamic equilibrium state of the alloy (solid curves), and the nonequilibrium uniform alloy composition (dotted curves). (b) The corresponding compositional profiles at steady state. The vertical line indicates the boundary between the first and second atomic layers. The migration energies of vacancies and interstitials via $\mathrm{Cu}$ and $\mathrm{Ni}$ atoms are tabulated. The symbols are used simply to label the curves for different temperatures. (from ref. 80)

Experimental evidence of these observations comes from an experiment conducted by Rehn et al. [103] in which a $\mathrm{Cu}-40$ at\% $\mathrm{Ni}$ alloy was bombarded by $5 \mathrm{keV} \mathrm{Ar}$ ions at elevated temperatures. AES and room temperature ion sputtering were used to reveal two different subsurface regions in which nickel enrichment was observed, both of which decay exponentially with depth. Region I has a decay length between 20 and $40 \mathrm{~nm}$ and region II has a decay length up to $1600 \mathrm{~nm}$. However, calculated values of the decay lengths were smaller than the experimental values by factors ranging from approximately 3 to 8 . Nevertheless, the fact that the calculations underestimate the RIS contribution very deep in the specimen while still predicting a substantial RIS contribution in the near-surface region suggests that RIS can play an important role in determining near-surface compositional changes during sputtering at temperatures where defects are mobile.

Shimizu [104], and Shimizu et al. [105-109] as well as others have measured the surface concentration of $\mathrm{Cu}-\mathrm{Ni}$ alloys as a function of sputtering time. With 
careful AES and ISS measurements, results have shown the existence of ion beam induced surface segregation such that the component with lower surface energy $(\mathrm{Cu})$ tends to segregate to the outermost atomic layer (GA). The result is enrichment of the atoms of lower surface energy at the surface and, at the same time, their depletion beneath the outermost atomic layer. This leads to the conclusion that radiation-induced surface segregation plays as substantial a role in the preferential sputtering of alloys as kinetic collision processes. Swartzfäger et al. [110] confirmed these findings at $200^{\circ} \mathrm{C}$ and Lam et al. [81] observed similar results over a range of temperatures, which are consistent with the picture that an increase in the $\mathrm{Cu}$ concentration of the first atomic layer due to Gibbsian adsorption is balanced by a corresponding decrease in the $\mathrm{Cu}$ concentration in the second layer.

In the $\mathrm{Au}-\mathrm{Cu}$ system, a similar result is found with preferential sputtering of $\mathrm{Cu}$ atoms producing an enrichment of $\mathrm{Au}$ at the the outermost atomic layer and $\mathrm{Au}$ depletion beneath the outermost atomic layer [111-113]. The factors causing preferential sputtering are differences in both the masses and surface binding energies of the constituent atoms, as well as radiation-induced surface segregation as shown in Fig. 38. In $\mathrm{Au}-\mathrm{Cu}$, the mass effect plays a dominant role to establish the surface composition, followed by the surface segregation of $\mathrm{Au}$ with its lower binding energy, leading to a stable state when equilibrium is reached. However, surface segregation is considered to be more dominant in $\mathrm{Cu}$ $\mathrm{Ni}$ alloys. Note that the depth scale of these processes is orders of magnitude shallower than that for RIS.

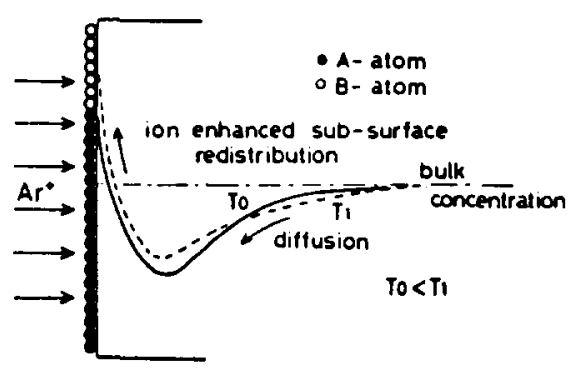

Fig. 38. Schematic illustration of the formation of an altered layer on the sample surface under ion bombardment. Solid and dotted curves indicate the composition vs depth profiles for two different temperatures, $T_{0}$ and $T_{1}$. (from ref. 104) 
Wiedersich et al. [98] provided a nice description of the effects of the different processes on the time evolution of the surface concentration and on the steady state profiles in a $\mathrm{Cu}-40$ at\% $\mathrm{Ni}$ alloy bombarded by $5 \mathrm{keV} \mathrm{Ar}^{+}$at $400^{\circ} \mathrm{C}$, as illustrated in Figs. 39 and 40 . The calculations were performed with various combinations of preferential sputtering, displacement mixing, radiationenhanced diffusion, Gibbsian adsorption and radiation-induced segregation included. Figure 39 shows the time dependence of the $\mathrm{Cu}$ concentration at the alloy surface, calculated at $400^{\circ} \mathrm{C}$ [114]. Note that in the absence of irradiation (curve \#1), GA leads to a strong $\mathrm{Cu}$ enrichment in the first atom layer. Accounting only for PS and RED during irradiation (\#2) causes a monotonic decrease in $\mathrm{C}_{\mathrm{Cu}}$ s to the steady state value, defined by the sputtering probability ratio and the bulk composition. If $\mathrm{GA}$ is included (\#3), $\mathrm{C}_{\mathrm{Cu}}$ s increases rapidly at short times owing to radiation-enhanced adsorption, and then decreases slowly to the steady state value. The inclusion of DM reduces the effect of GA (\#4). Considering only PS, RED and RIS (\#5), $\mathrm{C}_{\mathrm{Cu}}$ s decreases rapidly to the steady state value due to the sominant effect of segregation. If GA is added (\#6) then the effect of RIS is masked. Finally, with the addition of DM (\#7), or when all processes are included, $\mathrm{C}_{\mathrm{Cu}}$ s increases initially and then decreases toward the

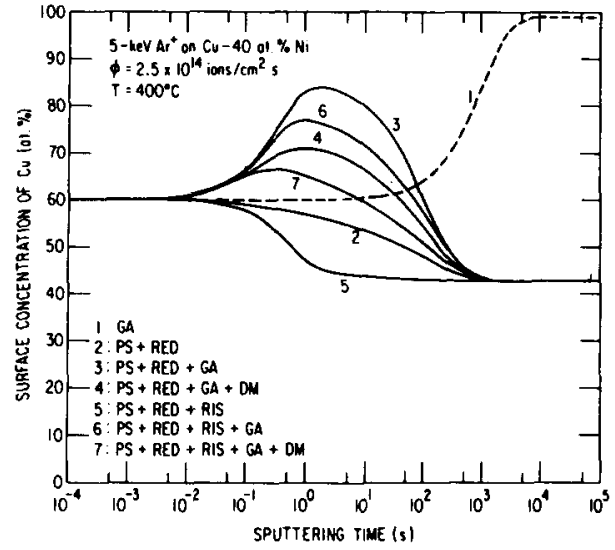

Fig. 39. Effects of various combinations of Gibbsian adsorption (GA), preferential sputtering (PS), radiation-enhanced diffusion (RED), displacement mixing (DM), and radiation-induced segregation (RIS) on the time evolution of the surface concentration of $\mathrm{Cu}$ during sputtering at $400^{\circ} \mathrm{C}$. (from ref. 98)

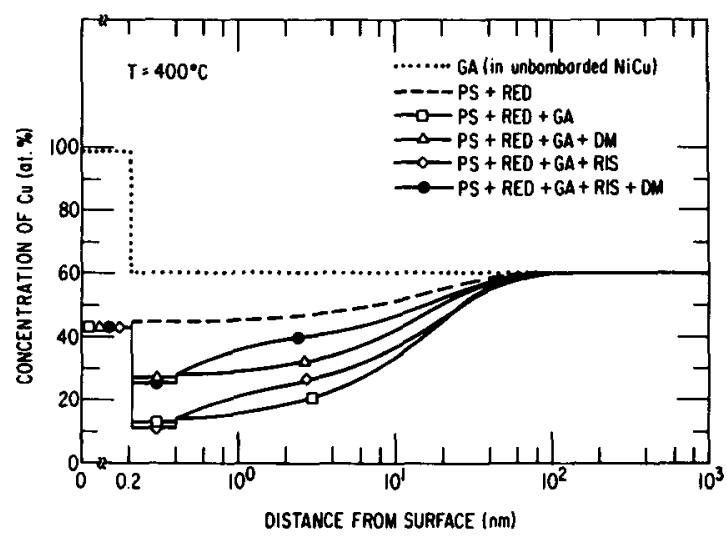

Fig. 40. Effects of various combinations of the five basic processes defined in Fig. 39 on the steady-state $\mathrm{Cu}$ concentration profiles during sputtering at $400^{\circ} \mathrm{C}$. (from ref. 98) 
steady state value. The effect of different combinations of processes on the steady-state $\mathrm{Cu}$ concentration profile is illustrated in Fig. 40.

\section{Implant redistribution during ion implantation}

A kinetic model has been developed recently by Lam and Leaf [115] to describe the effects of these kinetic processes on the spatial redistribution of implanted atoms during the implantation process. The effects of spatially nonuniform rates of damage and ion deposition, as well as the movement of the bombarded surface as a result of sputtering and introduction of foreign atoms into the system, were taken into account. The evolution of the implant concentration profile in time and space was calculated for various temperatures, ion energies, and ion-target combinations for a metal substrate $B$ into which $A$ atoms are implanted at a flux $\phi$. The local concentrations of vacancies (v), B interstitials (iB), A interstitials (iA), A-vacancy complexes (vA) and free substitutional solutes (A) change with implantation time according to a system of kinetic equations [80] similar to those of eqns. (3.78) through (3.80).

Concurrently with the buildup of solute concentration in the host matrix, the surface is subjected to displacements both from sputtering and the introduction of foreign atoms into the system. Sputtering causes a recession of the surface while implantation causes an expansion. The net surface displacement rate is controlled by the competition between the rates of ion collection and sputtering.

The temporal and spatial evolution of the surface and subsurface alloy composition is obtained by solving this set of equations for a semi-infinite medium, starting from the thermodynamic equilibrium conditions. Sample calculations [115] were performed for low and high-energy $\mathrm{Si}^{+}$and $\mathrm{Al}^{+}$ implantations into $\mathrm{Ni}$, since it is known from earlier studies that in irradiated $\mathrm{Ni}$, $\mathrm{Si}$ segregates in the same direction as the defect fluxes whereas $\mathrm{Al}$ opposes the defect fluxes [116]. Redistributions of $\mathrm{Al}$ and $\mathrm{Si}$ solutes in $\mathrm{Ni}$ during $50 \mathrm{keV}$ implantation at $500^{\circ} \mathrm{C}$ are shown in Figs. 41 and 42 , respectively.

In the Al-implantation case, $\mathrm{C}_{\mathrm{Al}} \mathrm{s}$ increases with time to a steady state value of $\sim 50$ at $\%$, which is governed by the partial sputtering yield of the implant. This value is substantially larger than that obtained in very high energy implantation, where sputtering is negligible and $\mathrm{C}_{\mathrm{Al}} \mathrm{s}$ is controlled by RIS. However, the total implant concentration remaining in the sample is significantly smaller because of sputtering. Furthermore, the shape of the steady state implant profile is dictated by PS which controls the boundary condition at the surface, and by RED and RIS. 


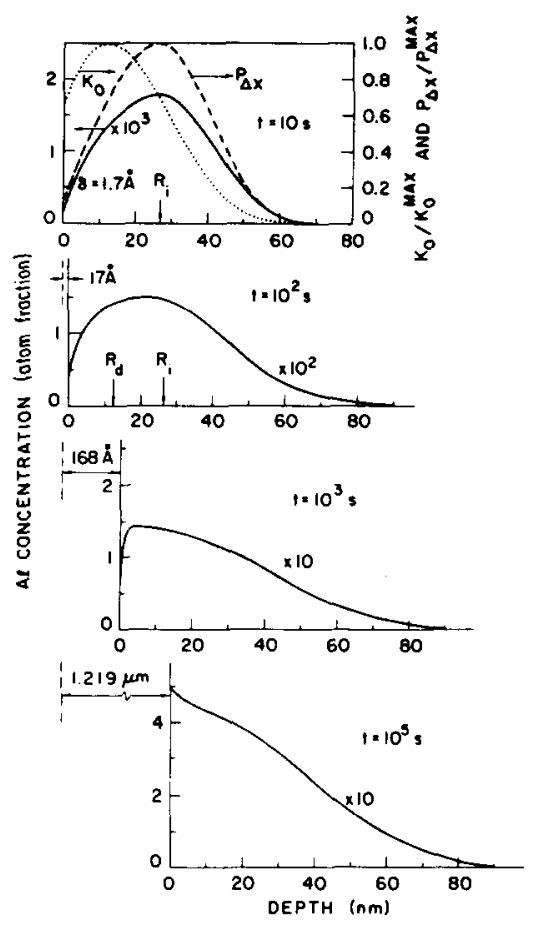

Fig. 41. Development of the Al profiles during $50-\mathrm{keV}$ implantation at $500^{\circ} \mathrm{C}$. The normalized damage $\left(\mathrm{K}_{0}\right)$ and ion-deposition $\left(\mathrm{P}_{\Delta \mathrm{x}}\right)$ rates are shown in the top portion, and the surface displacements resulting from sputtering are indicated. Note that the concentration scales are multiplied by factors shown in each plot. (from ref. 80 )

The evolution of the $\mathrm{Si}$ profile is rather different from that of $\mathrm{Al}$, because of the different RIS behaviors. After a short implantation time, Si enrichment occurs at the surface because of RIS, and the Si distribution peak starts moving into the sample interior, Fig. 42. With increasing time, $\mathrm{C}_{S \mathrm{i}}$ s increases monotonically, attaining a steady state value of $\sim 100$ at $\%$ at $\geq 2 \times 10^{4} \mathrm{~s}$. Unlike the Al case, the Si profile shows a significant shift of the implant distribution into the beyond-range region. This predicted translation of the Si-distribution peak into the sample interior was consistent with recent experimental measurements by Mayer et al. [117] in Si-implanted $\mathrm{Ni}$ at elevated temperatures. 


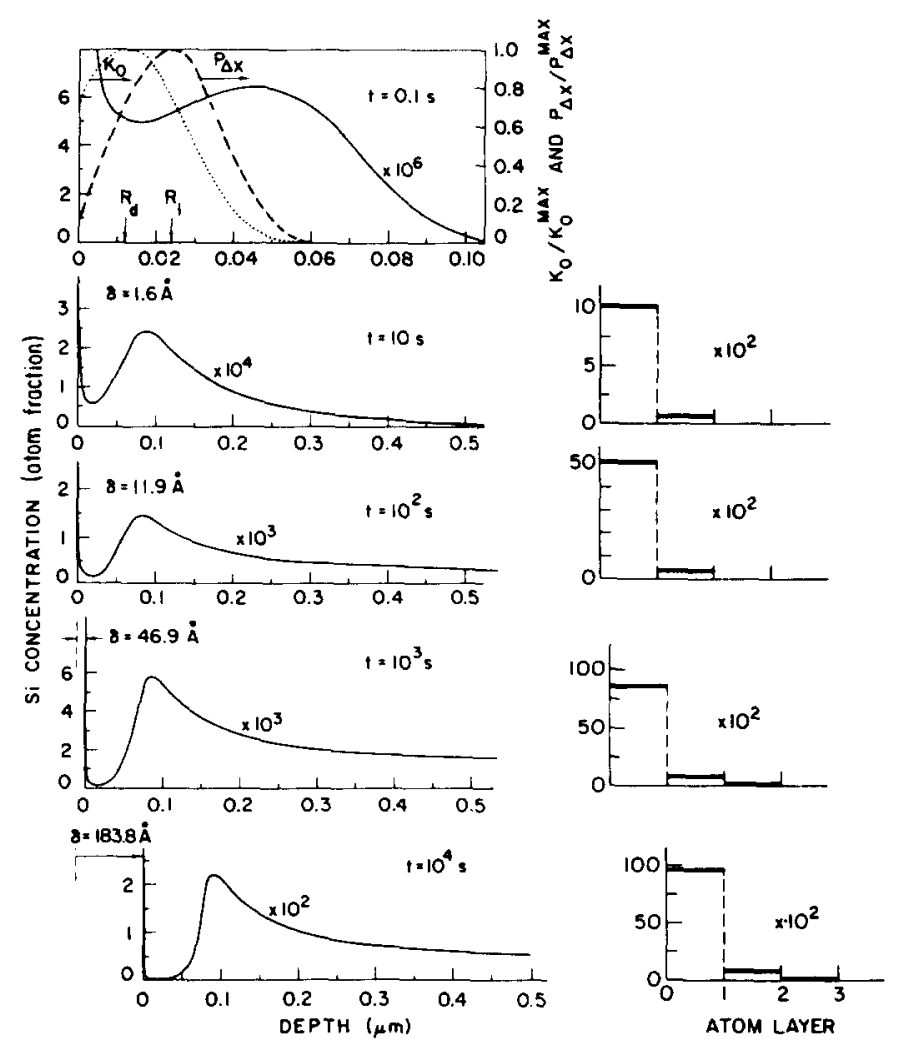

Fig. 42. Time evolution of the Si profiles during $50-\mathrm{keV}$ implantation at $500^{\circ} \mathrm{C}$. The normalized damage and ion deposition rates are shown in the top portion. Surface displacements $d$ resulting from sputtering are indicated. The rapidlychanging $\mathrm{Si}$ concentrations in the first three atomic layers are shown in the right inserts (each layer is $0.203 \mathrm{~nm}$ thick). (from ref. 80)

\section{Microstructural Changes}

Central to the changes occurring in alloys under irradiation is the alteration of the phase microstructure. This includes radiation-induced precipitate nucleation, precipitate growth and stability, resolution, phase redistribution and changes in phase composition. These processes fall under the category of phase stability. The subject of phase stability under irradiation has been studied for many years and has its origins in the U. S. breeder reactor program. The topic 
has been the subject of numerous excellent and recent reviews [57,118-122] Due to the nature of its origin, the emphasis has been on the reaction of the microstructure to the deposited energy (typically from neutrons) and less on the role of the bombarding particle or the change in composition during irradiation. The commonality with ion bombardment is in the damage introduced during the irradiation. However, there are a number of additional processes which affect the microstructure which are more specific to ion implantation, ion beam mixing or ion beam assisted deposition, such as metastable phase formation, ioninduced grain growth, etc. Consequently, this section is divided into three parts. Part A briefly summarizes the state of our knowledge in phase stability under irradiation, part $B$ discusses the formation of metastable phases by ion implantation or ion beam mixing, and part $\mathrm{C}$ describes additional aspects of microstructural evolution during ion irradiation.

\section{A. Phase stability}

The stability of precipitates under irradiation is a consequence of the processes discusses in section 3. Irradiation affects precipitate stability through an increased defect concentration, enhanced diffusion, segregation, and ballistic processes. The increased atomic mobility can enhance the rate of formation or resolution of a precipitate. The segregation of a solute to a sink can induce local compositional changes which are large enough to change the local phase equilibrium resulting in precipitate nucleation or dissolution. Vacancies and interstitials may also have a direct effect on nucleation of precipitate phases, by accommodating volume expansion or contraction at the nucleus interface. Displacement events may cause precipitate shrinkage by physically ejecting (recoil resolution) atoms from the interior to the matrix. The recoil resolution will be opposed by a diffusive flux from the matrix to the particle and the balance between these two processes will then determine the degree to which irradiation will destabilize the precipitate. Irradiation may also disorder regions in a thermodynamically stable ordered precipitate giving rise to their dissolution by virtue of an increased solubility of the disordered region. Finally, precipitates may be nucleated under irradiation. The following discussion begins with a treatment of radiation-induced nucleation of precipitates.

(i) Precipitate nucleation. Katz and Wiedersich [123] developed an expression for the nucleation rate of precipitate nuclei at steady state which was directly 
proportional to the arrival rate, $\beta_{\mathrm{e}}$ of solute in the saturated solution and, hence to the radiation-enhanced diffusion coefficient,

$$
J=\beta_{e} f(1) / \eta_{e}(1)\left\{\Sigma\left[s(x) \eta_{e}(x)\left(C / C_{e}\right)^{x}\right]^{-1}\right\}^{-1}
$$

where $f(x)$ is the concentration of embryos containing $x$ atoms per unit area, $\eta_{e}(x)$ is the equilibrium concentration of embryos in the saturated solid solution, $s(x)$ is the surface area of an embryo of $x$ atoms, and $C / C_{e}$ is the ratio of solute concentrations in the supersaturated and saturated solid solution. Note that at steady state, $J$ is independent of both $x$ and $t$.

Mruzik and Russell [124] developed a formulation for the nucleation rate applicable to calculation of the rate of incoherent precipitate nucleation in irradiated metals. The nulcei are taken as spherical and of elemental composition. Each particle is characterized by the number of atoms it contains, $x$, and by the number of excess vacancies it contains, $n$. Processes giving rise to changes in the state of the particle are those involved in growth plus the possibility of a nucleus being struck by a displacement cascade and dispersed into single solute atoms, vacancies, and interstitials. The calculation focuses on the number of nuclei growing past a certain value of $x$, per unit of volume and time. At steady state, this nucleation flux is independent of $x$ and is given by

$$
\mathrm{J}_{\mathbf{S}}(\mathrm{x})=\Sigma\left[\beta_{\mathbf{x}} \rho(\mathrm{n}, \mathrm{x})-\alpha_{\mathrm{x}}(\mathrm{n}, \mathrm{x}) \rho(\mathrm{n}, \mathrm{x}+1)\right]
$$

where $\beta_{x}$ is the arrival rate of solute to the embryo, $\alpha_{x}$ is the loss rate of solute from the embryo, and $\rho(n, x)$ is the number of particles of $n$ excess vacancies and $x$ solute atoms, per unit volume of material. The $\beta$ 's are determined by the concentrations and mobilities of the respective point defects and the time rate of change of $\rho(n, x)$ is obtained from a balance between particles entering and leaving a given $(n, x)$ size class.

Supersaturated vacancies were found to have a profound effect on enhancing precipitate nucleation. Furthermore, no reasonable values of solute supersaturation and interfacial energy will give an observable rate of nucleation in the absence of a vacancy supersaturation. While the presence of excess interstitials reduces the nucleation rate somewhat, the rate is still considerably higher than without vacancies. Frost and Russell [121] also showed that irradiation-induced vacancies stabilize particles for supersaturated, undersaturated or saturated solutions.

Whereas the increased nucleation rate resulting from the radiation-enhanced diffusion should be a general phenomenon, other effects of radiation on 
nucleation have been suggested. For example, misfit strains can be reduced by incorporation of excess point defects of the proper type into nuclei [125], as a consequence the equilibrium concentration, $\eta_{e}(x)$, of nuclei would increase and with it the nucleation rate. Cauvin and Martin [126] also suggested that enhanced vacancy-interstitial recombination at solute clusters may stabilize nuclei if solute segregation is associated with the defect flux to the clusters.

A very important and practical way in which precipitation can occur under irradiation is as a result of radiation-induced segregation [127]. As discussed in section 3.E, significant fractions of randomly produced defects annihilate at sinks, thus inducing defect fluxes. A preferential association or exchange of specific alloy components with defects couples net fluxes of alloy components to defect fluxes, which in turn alters the local composition near defect sinks. Usually, the matrix near sinks becomes depleted of the large atomic size components and enriched in the small atomic size components of the alloy. Local enrichments of solutes may be sufficiently large to exceed the solubility limit and precipitation can then occur in nominally solid solution alloys. Precipitation will continue until the matrix concentration decreases to a level that permits a sufficiently steep solute gradient to balance the defect flow-induced solute flux to the sinks by solute back diffusion. The temperature range in which radiationinduced precipitation is predicted to occur also goes through a maximum as a function of binding energy. The temperature range of precipitation shifts to lower temperatures with lower displacement rates, Fig. 43. A prime example is the precipitation of $\mathrm{Ni}_{3} \mathrm{Si}$ on surfaces, dislocation loops and on grain boundaries of a dilute $\mathrm{Ni}-\mathrm{Si}$ alloy [129].

The precipitation of ordered $\gamma^{\prime}\left(\mathrm{Ni}_{3} \mathrm{Si}\right)$ has also been reported in type 316 stainless steel after neutron irradiation below $\sim 525^{\circ} \mathrm{C}[130,131]$. This phase is not observed in 316 SS without irradiation and dissolves during prolonged postirradiation annealing [130,132]. The $\gamma^{\prime}$ phase is most likely a consequence of the strong segregation of $\mathrm{Si}$ and $\mathrm{Ni}$ to defect sinks. Sethi and Okamoto [133] have measured appreciable enrichment of $\mathrm{Si}$ and $\mathrm{Ni}$ and depletion of $\mathrm{Cr}$ in the near surface region of ion-irradiated austenitic $\mathrm{Fe}-\mathrm{Cr}-\mathrm{Ni}-\mathrm{Si}$ alloys. Neutron irradiations also induce precipitates of a ternary nickel silicide [130,131,134], Gphase and a silicon rich $\mathrm{M}_{6} \mathrm{C}$ [134]. Radiation-induced $\mathrm{G}$-phase but not $\gamma^{\prime}$ has been observed in ion irradiated type 316 stainless steel with $\mathrm{Si}$ and $\mathrm{Ti}$ modifications [135]. However, both phases are induced by $\mathrm{Ni}$-ion bombardment in austenitic $\mathrm{Fe}-12 \mathrm{wt} \% \mathrm{Cr}-15 \mathrm{wt} \% \mathrm{Ni}$ alloys with $\mathrm{Si}, \mathrm{Mn}$ and $\mathrm{Ti}$ additions [57]. High energy $\mathrm{Cr}+$ irradiation at $550^{\circ} \mathrm{C}$ of a ferritic steel containing $\mathrm{V}$, Mo or $\mathrm{Si}$ produce the $\mathrm{M}_{23} \mathrm{C}_{6}$ phase. Evidence exists to suggest that the incorporation of Mo and $\mathrm{V}$ into the $\mathrm{M}_{23} \mathrm{C}_{6}$ before irradiation may be a necessary prerequisite for 
measurable stability of this phase. In this alloy, all the grain boundary phases which precipitate upon heavy-ion irradiation are chromium-rich despite being formed at an interface where chromium depletion is occurring, suggesting that in this case the kinetics of precipitate formation are the dominant effect.

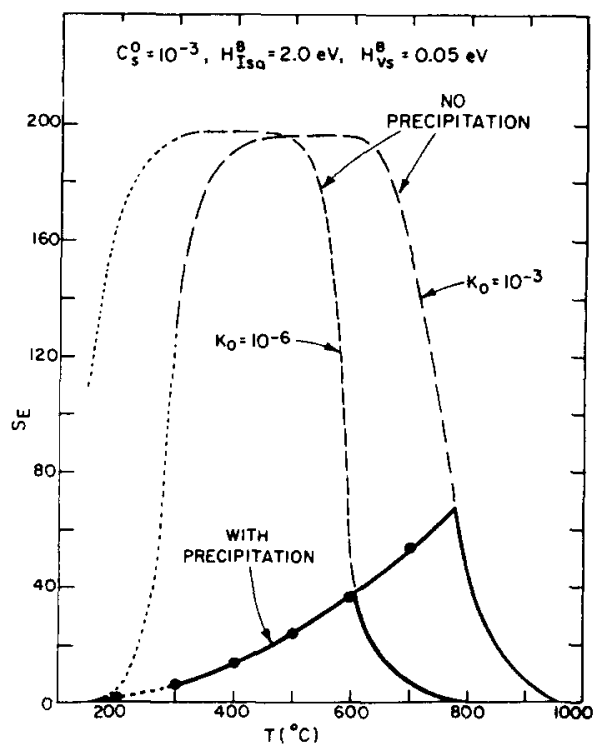

Fig. 43. Effect of defect-production rate on the temperature dependence of solute enrichment at a foil surface (no precipitation) and at the precipitatematrix interface (with precipitation). The parameters are chosen for nickel. (from ref. 128)

(ii) Precipitate growth and stability The earliest analysis of precipitate stability under irradiation was conducted by Nelson, Hudson and Mazey [136] who considered the balance between precipitate shrinkage due to irradiation resolution or disordering, and reprecipitation speeded by irradiation-enhanced diffusion. Particles are predicted to approach an equilibrium radius from larger or smaller size, at which point the rate of reprecipitation from the enriched matrix just balances the rate of solute loss from the particle due to disorder dissolution. This size dependence of particle stability is reversed from the usual, with smaller particles being more stable than larger particles. This is because dissolved material could also re-precipitate, at a rate governed by the irradiation-enhanced diffusion coefficient. The rate of mass loss of a particle of radius $r$ due to disordering was found to vary as $r^{2}$, and the rate of re- 
precipitation to vary as $r$. Thus, big particles were predicted to shrink and small particles to grow to a certain 'equilibrium' size as shown by the solid curve in Fig. 44. The model predicted that particles should approach the stable particle size after modest doses of a few dpa. The stable particle size was predicted to increase and the matrix solute concentration to decrease with increasing temperature.

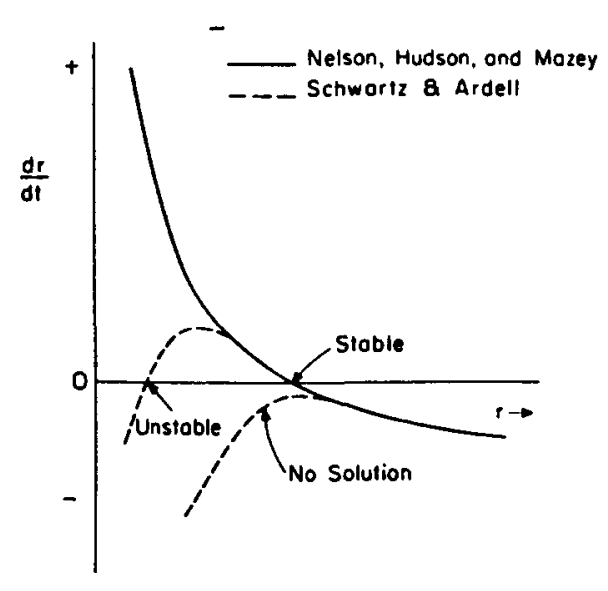

Fig. 44. Calculated growth rate for ordered, coherent particles undergoing irradiation-induced disorder dissolution. (from ref. 137)

Nelson did not, however, consider the increase in solubility which comes with a decrease in particle size and makes the smaller particles less stable. Russell [137] reported that Ardell and Schwartz accounted for the effect of curvatureenhanced solubility, with the results shown in Fig. 44. In some cases there is no 'equilibrium' size. Disorder-dissolution can reduce the particles to such a size that equilibrium solubility is high enough to put all particles back into solution. Thus, by dissolving the equilibrium phase, irradiation will have forced the system into such a state that a non-equilibrium phase could precipitate out. Subsequent analyses of this model [138-141] produce significantly different results including disagreements as to whether a unique steady state size distribution should be expected.

A theory for the stability of precipitates under irradiation was developed by Maydet and Russell [125]. They developed analytical expressions for the locations of nodal lines in $(n, x)$ space where the net rate of solute capture $(\dot{x})$ or of vacancy capture ( $\dot{n})$ is zero and for the critical point at which these lines 
intersect, Fig. 45 They found that a critical point exists only if $\Delta \phi<0$, where $\Delta \phi$ is an irradiation modified free energy which determines phase stability under irradiation,

$$
\begin{gathered}
\Delta \phi=-k T \ln S_{x}\left[S_{v}\left(1-\beta_{i} / \beta_{v}\right)\right]^{\delta}-k T\left[\ln S_{v}\left(1-\beta_{i} / \beta_{v}\right)\right]^{2} / 4 B \\
\text { where } \delta=(\Omega-\Omega m) / \Omega m \\
\text { and } B=\Omega E / 9 k T(1-v),
\end{gathered}
$$

and where $S_{\mathbf{x}}$ is the ratio of actual and saturation concentrations of solute, $S_{v}$ is the ratio of actual and saturation concentrations of vacancies, $\Omega$ is the atomic volume of the precipitate and $\Omega_{\mathrm{m}}$ is the atomic volume of the matrix, $E$ is Young's modulus and $v$ is Poisson's ratio. In that usually $\beta_{i} / \beta_{v}<1$, irradiation will tend to enhance the stability of a phase for which $\delta>0$, and destabilize in the reverse case. The former is usually the case since matrix solid solutions tend to be more closely packed than are precipitate phases.

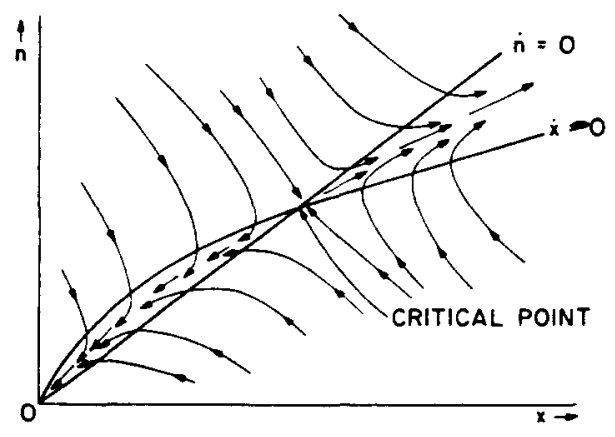

Fig. 45. Results of critical point/nodal line analysis of incoherent particle behavior under irradiation. (from ref. 137)

For an undersize precipitate phase $(\delta<0)$ to grow from a slightly supersaturated solid solution without prohibitive strain energy, the particle must emit vacancies into the matrix. In the absence of excess vacancies this is easily done, as vacancies arrive at and leave the interface at the same rate (in the absence of growth or decay), and there is no trouble in establishing a net flux out to allow growth. 
Under irradiation, the vacancy emission rate is not altered, but the arrival rate is increased by several orders of magnitude. It is then almost impossible to achieve a net emission of vacancies. There will instead tend to be a net gain of vacancies, resulting in a strain energy which is easiest relieved by emission of solute atoms in the face of the slight solute supersaturation. The excess vacancies thus destabilize the undersized precipitate phase. The same arguments may be used to understand stabilization of an oversized precipitate.

Cauvin and Martin [142] reanalyzed Russell's model for the growth of incoherent precipitates and showed that indeed, it qualitatively accounts for precipitation in the case of undersized precipitate atomic volume. More importantly, they observed that under appropriate conditions, undersaturated Al-Zn solid solutions give rise to a homogeneous precipitation of clusters which they argue to be Guiner-Preston zones of incoherent $\mathrm{Zn}$ precipitates, the atomic volume of which is smaller than that of the matrix.

They conducted a systematic TEM study of $1 \mathrm{MeV}$ electron irradiation damage in $\mathrm{Al} 1.9$ at $\% \mathrm{Zn}$ solid solution over a wide range of irradiation fluxes and temperatures, and showed that $\mathrm{Zn}$ precipitates form under irradiation at temperatures well above the $\mathrm{Zn}$ solvus temperature outside irradiation. This upward shift of the $\mathrm{Zn}$ solvus temperature under irradiation is dose rate dependent, thus defining a temperature dependent dose rate threshold for the occurrence of $\mathrm{Zn}$ precipitation in $\mathrm{AlZn}$ undersaturated solid solutions. The authors [142] suggest that an attractive solute-defect interaction in conjunction with defect-induced solute fluxes in the same direction as that of the defects, stabilize solute clusters present as a consequence of concentration fluctuations. Because of the defect-solute attraction, the defect concentration in solute rich clusters is enhanced. This leads to higher recombination losses within the clusters, which thus act as defect sinks. The clusters then grow by radiationinduced segregation.

The discovery of irradiation-induced precipitation in undersaturated solid solutions discredited the simple picture of the effects of irradiation on phase stability which described the phenomenon as simply resulting from a balance between radiation-induced disordering and radiation-accelerated diffusion towards the equilibrium state. Indeed it was learned that the solubility limit is dose rate dependent. Irradiation-induced precipitation is a result of radiationproduced point defects, and it is the supersaturation of these defects which provides the driving force for precipitation. For a given irradiation temperature and dose rate, the point defect supersaturation in the solid solution under irradiation is a function of the solute content and of the point defect sinks and trap density. Once precipitates are formed, they act as traps or sinks for point 
defects. The point defect supersaturation in the matrix is therefore lowered by the presence of the precipitates; precipitation will cease before the solute content of the matrix has reached the solubility limit without precipitates.

(iii) Coarsening. The theory for thermal coarsening was developed by Lifschitz and Slyozov [143], and Wagner [144]. Here, particles with a diameter larger than the mean diameter grow under the driving force of the interfacial free energy at the expense of particles smaller than the mean diameter. During coarsening, the precipitate size distribution and volume fraction remain essentially unchanged while the mean size increases such that $\mathrm{r}^{3}-\mathrm{r}_{0}{ }^{3}=\mathrm{Kt}$ where $\mathrm{K} \alpha \mathrm{D}$, the diffusion coefficient. Precipitate coarsening under irradiation has been studied by a number of authors [138-141]. The main effect of irradiation is the acceleration of the rate of particle growth due to radiation-enhanced diffusion. This has been confirmed for $\mathrm{Ni}-12.8$ at\% $\mathrm{Al}[145,146]$ and $\mathrm{Ni}-12.7$ at\% $\mathrm{Si}[147,148]$ as will be discussed in section 4.A.vi.

Baron et al. [139] considered the effects of irradiation resolution and enhanced diffusion, and allowed the matrix concentration to rise above the thermal value. Particles were allowed to change size by two processes: (1) steady drift due to the rates of thermal and irradiation resolution being greater (or less) than the rate of reprecipitation from the matrix, and (2) random walk due to statistical fluctuations in the rates of solute addition and loss. For particles which are initially larger than the maximum stable size, particle shrinkage $r$ varies linearly with time, with the proportionality constant depending only on the irradiation parameters. However, for initial particle sizes much smaller than the stable size, the coarsening has an $r^{3}$ versus $t$ dependence that is very similar to that for thermal coarsening and the proportionality coefficient in both cases involves the diffusion coefficient.

Urban and Martin [149] developed a theory for precipitate coarsening that includes point defect recombination at precipitate interfaces as an additional driving force. When a defect of opposite type as those already trapped arrives at the particle, it annihilates immediately so that three types of precipitates coexist: those with trapped vacancies, those with trapped interstitials and those without trapped defects. Because of the irreversibility of recombination, net fluxes of defects to the precipitate matrix interfaces are set up which affect the coarsening kinetics if radiation-induced segregation occurs. They have shown that for a system such as a dilute Al-Zn alloy, in which vacancies are trapped preferentially at the interface and in which the $\mathrm{Zn}$ solute segregates in the same direction as the defect fluxes, the coarsening kinetics is accelerated and the size 
distribution widened initially during irradiation, but both approach their purely radiation-enhanced coarsening values at long times.

(iv) Precipitation of implanted gases. Another example of precipitate stability due to implantation was discovered in 1984 when heavy inert gases ( $\mathrm{Ar}$ and $\mathrm{Xe}$ ) were implanted into aluminum and precipitated in a solid form [150,151]. Electron diffraction studies show identical symmetry of the rare gas diffraction pattern and of the host metal diffraction pattern indicating epitaxial alignment of rare gas crystallites and host matrix. For the lighter rare gas $\mathrm{Ne}$, the occurrence of liquid $\mathrm{Ne}$ precipitates under high pressures in implanted metals at room temperature has been reported [150,152]. For the lightest rate gas $\mathrm{He}$, evidence for solid $\mathrm{He}$ in small cavities at room temperature has been obtained for implanted $\mathrm{Ni}$ [153]. These results demonstrated that inert gas bubbles in metals formed by implantation at ambient temperatures are under high pressures, of the order of several GPa.

Recently, Birtchaer and Jäger [154] performed a careful study of the microstructural changes and the precipitation of $\mathrm{Kr}$ in thin films of $\mathrm{Al}$ during 65 $\mathrm{keV} \mathrm{Kr}^{+}$implantation at room temperature. Their TEM observations show that $\mathrm{Kr}$ becomes trapped in a dense population of growing cavities. At low fluences ( $\leq$ $10^{15} \mathrm{Kr}^{+} / \mathrm{cm}^{2}$ ) dislocation loops and the formation of a dislocation network is observed. The loops increase in size as the $\mathrm{Kr}$ fluence is increased until $\left(2 \times 10^{14}\right.$ $\mathrm{cm}^{-2}$ ) their interaction results in the formation of a dislocation network. At high fluences $\left(\geq 10^{15} \mathrm{~cm}^{-2}\right.$ the microstructure is dominated by a high concentration of cavities with larger cavities on the grain boundaries. At a fluence of $1.3 \times 10^{16}$ $\mathrm{cm}^{-2}$, the single crystal Al grains contain a high density $\left(1 \times 10^{20} \mathrm{~cm}^{-3}\right)$ of cavities whose average diameter is $1.7 \mathrm{~nm}$ with sizes up to $2.7 \mathrm{~nm}$. Increasing the $\mathrm{Kr}$ fluence to $2.2 \times 10^{16} \mathrm{~cm}^{-2}$ results in an increase of the average cavity diameter to $2.8 \mathrm{~nm}$ with a maximum diameter of $5 \mathrm{~nm}$, and a decrease of the cavity density to $0.5 \times 10^{20} \mathrm{~cm}^{-3}$. At a fluence of $5.5 \times 10^{16} \mathrm{~cm}^{-2}$, the size distribution contains cavities as large as $40 \mathrm{~nm}$. In all cases, grain boundary cavities were much larger than the largest cavities within the grains [155]. The authors suggest that the formation of visible cavities containing the implanted $\mathrm{Kr}$ can occur by the growth of Kr-vacancy complexes due to $\mathrm{Kr}$ absorption. $\mathrm{Kr}$ transport to cavities could result from diffusion by vacancy or divacancy mechanisms.

Evans [156] notes, however, that there appears to be general agreement that the result can only be consistent with a pressure-driven cavity growth process such as loop punching [157] in which a cavity can gain vacancies at the expense 
of punching out interstitial loops into the matrix. This view is supported by a recent compilation of results for krypton and xenon in a number of metals which showed that the pressure of the solid inert-gas precipitates was a strong function of the metal shear modulus [158].

(v) Order-disorder transformations. The effect of irradiation on ordered alloy phases can be described as a balance between two conflicting processes: the disordering produced by the atomic displacements during defect production and the ordering facilitated by the thermal migration of excess vacancies and interstitials [159-161]. The rate of change of the order parameter, $S$, is the sum of the irradiation disordering and radiation-enhanced thermal ordering rates:

$$
\mathrm{dS} / \mathrm{dt}=(\mathrm{dS} / \mathrm{dt})_{\mathrm{irr}}+(\mathrm{dS} / \mathrm{dt})_{\mathrm{th}}
$$

The disordering rate depends on the rate of atom replacement, which should be linear with the irradiation flux. If the atoms are replaced randomly by other atoms, we find that the disordering rate is proportional to the order parameter:

$$
(\mathrm{dS} / \mathrm{dt})_{\mathrm{irr}}=\varepsilon K S,
$$

where $\varepsilon$ is the disordering efficiency, which depends on the type of radiation. Note that this approximation shows no dependency on temperature.

The ordering rate is proportional to the concentration of mobile defects, the rate at which they jump, and the fraction by which ordering jumps exceed disordering jumps. The ordering rate therefore depends on the radiation flux through the dependence on defect concentration. It depends on temperature through the defect concentration and the jump rate and jump preference terms. As the temperature is lowered, the fraction of ordering jumps should increase because of an increasingly favorable Arrhenius factor. Therefore, in the regime where diffusion to fixed sinks dominates, the ordering rate should increase as the temperature is lowered. Only when the temperature is reached at which direct recombination of defects becomes important will the trend reverse. However, at temperatures where vacancies are immobile, even the enhanced ordering rate is insignificant and the disordering process will dominate. At higher temperatures where the thermal equilibrium vacancy concentration is large, there is little enhancement of the thermal ordering rate by irradiation. As a function of dose, the steady-state ordered and disordered states shift to lower temperature with lower dose rates. 
Experimental evidence is in good agreement with theory in many cases. For example, Banerjee et al. [162] have shown that the long-range order in $\mathrm{Ni}_{4} \mathrm{Mo}$ remains at the equilibrium order parameter above $520 \mathrm{~K}$ during irradiation at 5 $x \quad 10^{-3} \mathrm{dpa} / \mathrm{s}$. Below that temperature, the steady state long-range order decreases rapidly with irradiation temperatures becoming essentially zero below 450K. The decrease in order at low temperatures is exponential with dose. More examples of irradiation disordering are provided in section 4.B.i.

(vi) Phase redistribution and composition modifications. Phase redistribution has been frequently reported, but only a few systematic investigations exist. The most extensive studies have been reported by Potter and coworkers in a Ni12.8 at $\% \mathrm{Al}$ alloy $[145,146]$ and in a $\mathrm{Ni}-12.7$ at\% $\mathrm{Si}$ alloy $[147,148]$. Both alloys are two-phase in the temperature range of the $\mathrm{Ni}$-ion bombardment investigated $\left(400-700^{\circ} \mathrm{C}\right)$. Solution annealed and quenched samples of $\mathrm{Ni}-12.8$ at $\% \mathrm{Al}$ were irradiated with $\mathrm{Ni}$ ions at intermediate temperatures. Irradiation at low doses [153] causes the formation of a uniform distribution of small $\gamma^{\prime}$ precipitates except near interstitial dislocation loops and other sinks, Fig. 46. As the dislocation loops grow with increasing dose, the precipitate-free zones (PFZ) enclosing the loops grow correspondingly by dissolution of the precipitates because of radiation-induced segregation of $\mathrm{Ni}$ into these regions. The precipitates in the sink-free regions between PFZs coarsen at a radiationenhanced rate. At higher doses $\left(\geq 15 \mathrm{dpa}\right.$ at $\left.550^{\circ} \mathrm{C}\right), \gamma^{\prime}$-precipitates renucleate within the PFZ colonies. Apparently the continued drain of $\mathrm{Ni}$ toward the loop perimeter reverses the initial Ni-enrichment in regions of the zones that become remote from the dislocations, and ultimately reprecipitation of $\mathrm{Ni}_{3} \mathrm{Al}$ occurs there. A consequence of the reprecipitation process is a decrease in the average precipitate size during prolonged irradiation.

While radiation-induced segregation in two-phase $\mathrm{Ni}-\mathrm{Al}$ alloys promotes the single phase $\gamma$ solid solution in regions near sinks, the opposite occurs in twophase Ni-Si alloys [147,148], Fig. 47. In this case, low doses causes the formation of small $\gamma^{\prime}\left(\mathrm{Ni}_{3} \mathrm{Si}\right)$ precipitates in the initially solution-quenched alloy. At higher dose, disk-like precipitates grow in thickness and diameter with the growing dislocation loops, consuming nearly all the $\gamma^{\prime}$ in the matrix. At grain boundaries and at surfaces, continuous $\mathrm{Ni}_{3} \mathrm{Si}$ films grow during irradiation at the expense of the precipitates in the interior of grains [148,163]. 


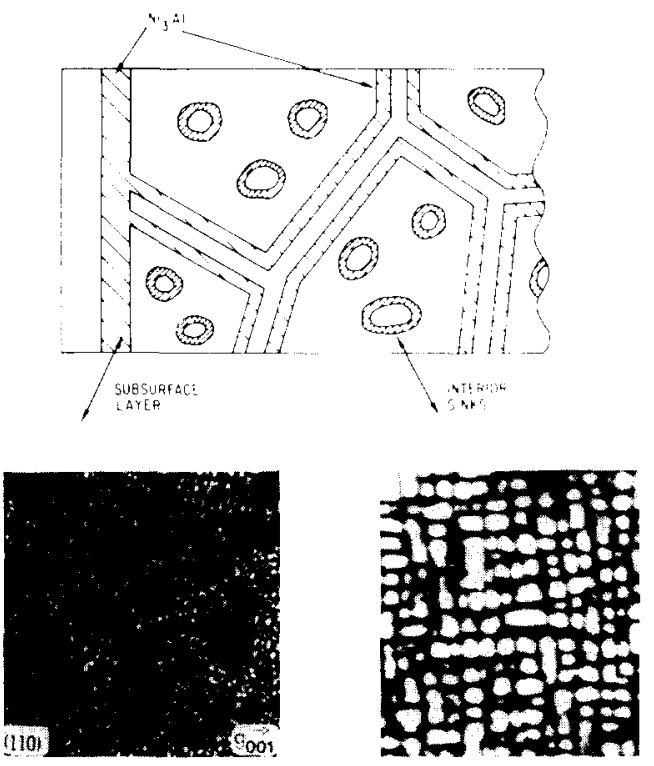

Fig. 46. Formation of $\gamma^{\prime}-\mathrm{Ni}_{3} \mathrm{Al}$ away from defect sinks in a solid solution $\mathrm{Ni}-\mathrm{Al}$ alloy because of RIS. The dark field micrographs from a two-phase $\mathrm{Ni}-\mathrm{Al}$ alloy show (a) $\gamma^{\prime}$ particles concentrated near the center of a thin foil and (b) preferential formation of $\gamma^{\prime}$ particles away from interstitial loops during ion bombardment. (from ref. 62)
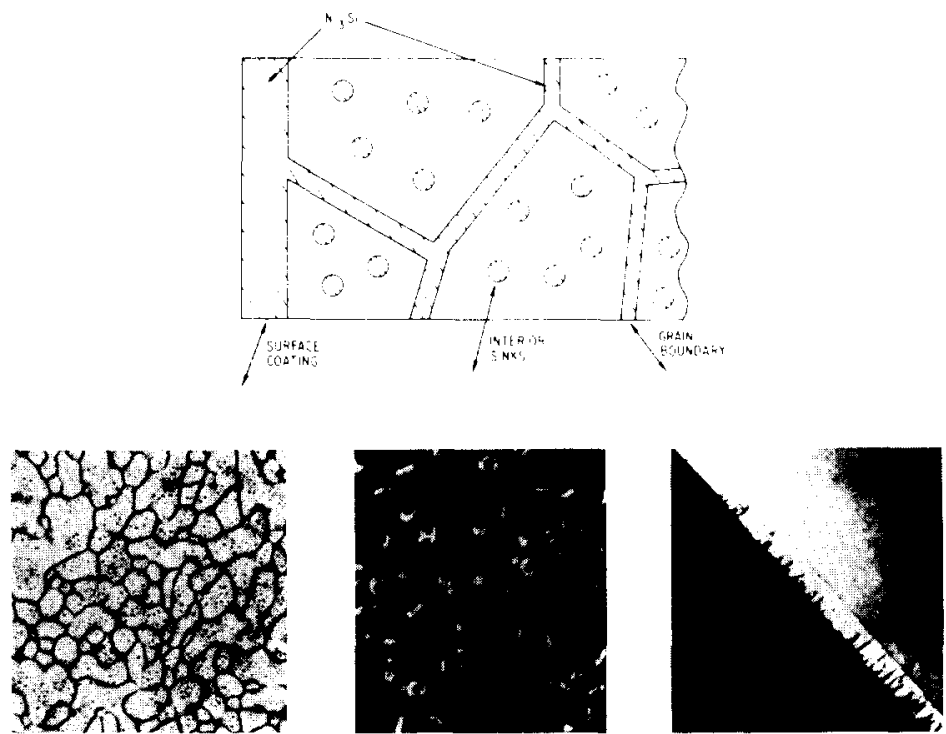

Fig. 47. Formation of $\gamma^{\prime}-\mathrm{Ni}_{3} \mathrm{Si}$ on defect sinks in a solid solution $\mathrm{Ni}-\mathrm{Si}$ alloy because of RIS. The dark-field micrographs show (a) the anti-phase domain structure in a contiguous surface coating: (b) toroidal $\gamma^{\prime}$ precipitates on interstitial loops: and (c) a grain boundary coated with $\gamma^{\prime}$. (from ref. 62) 
In addition to changes in the phase distributions of an alloy under irradiation, significant changes can occur in the composition of the phases. Wiedersich [118] provides an explanation for such an occurrence. At thermal equilibrium, the compositions on both sides of the interface between matrix and a second phase precipitate are uniquely defined. As long as equilibration can quickly occur across the interface, this region will adjust so that adjacent matrix precipitate compositions correspond to those of the equilibrium phase diagram. Thus, excess solute carried by defect fluxes from the matrix to a precipitate interface is accommodated by growth of the precipitate at a composition determined by the phase diagram. In binary alloys, this composition is a function of temperature alone and, to the degree that the composition within the precipitates can be considered spatially constant, the precipitates are the equilibrium phase.

In multicomponent alloys, the compositions of second phase precipitates can be altered significantly from those present in the alloy at thermal equilibrium by radiation-induced segregation. An example is the increased $\mathrm{Ni}$ and $\mathrm{Si}$ concentrations and the decreased $\mathrm{Cr}$ concentration at defect sinks by radiationinduced segregation in austenitic alloys $[133,134]$. If persistent defect fluxes from the matrix to second phase precipitate interfaces are maintained during irradiation, the precipitates will become enriched in $\mathrm{Ni}$ and $\mathrm{Si}$ and depleted in $\mathrm{Cr}$. Thus, Wiedersich points out [118] that the additional degrees of freedom in multicomponent alloys permit, even in the absence of concentration gradients within the precipitates, the compositions of second phases to become significantly different during irradiation from those in the unirradiated alloy.

Williams, Boothby and Titchmarsh [134] conducted a study of four $12 \mathrm{Cr}-15 \mathrm{Ni}$ austenitic alloys containing silicon in the range 0.14 to $1.42 \mathrm{wt} \%$ that were irradiated with neutrons to a dose of approximately $20 \mathrm{dpa}$ at temperatures in the range $400^{\circ} \mathrm{C}$ to $645^{\circ} \mathrm{C}$. After irradiation, only the low silicon alloy remained predominantly austenitic. At high temperatures the transformation to ferrite was confined to the grain boundary whereas at lower temperatures the transformation often extended throughout the grain. Nickel and silicon segregate to, and chromium and iron are depleted at grain boundaries. In addition, intragranular regions separate into nickel and silicon rich, chromium and iron depleted, and chromium and iron rich, nickel and silicon depleted zones, Fig. 48. At the highest irradiation temperatures, the silicon remains in solution in silicon rich intragranular regions, and chromium rich, nickel depleted regions remain austenitic. The scale of the compositional variations is on the order of $700 \mathrm{~nm}$. However, at lower temperatures silicon rich precipitates are formed 
within the silicon rich regions and the chromium rich regions transform toferrite. The wavelength of the compositional oscillation is considerably shorter, on the order of 25-50 nm, Fig. 49.

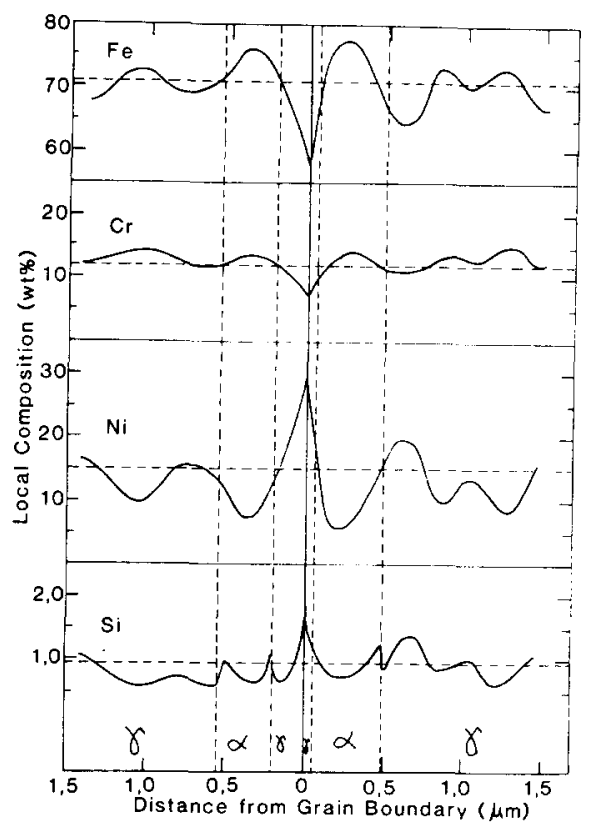

Fig. 48. Compositional variation near a grain boundary in a $12 \mathrm{Cr}-15 \mathrm{Ni}$ alloy containing $0.95 \% \mathrm{Si}$ after irradiation to $23.6 \mathrm{dpa}$ in $\mathrm{EBR}$ II at $645^{\circ} \mathrm{C}$. (from ref. 134)

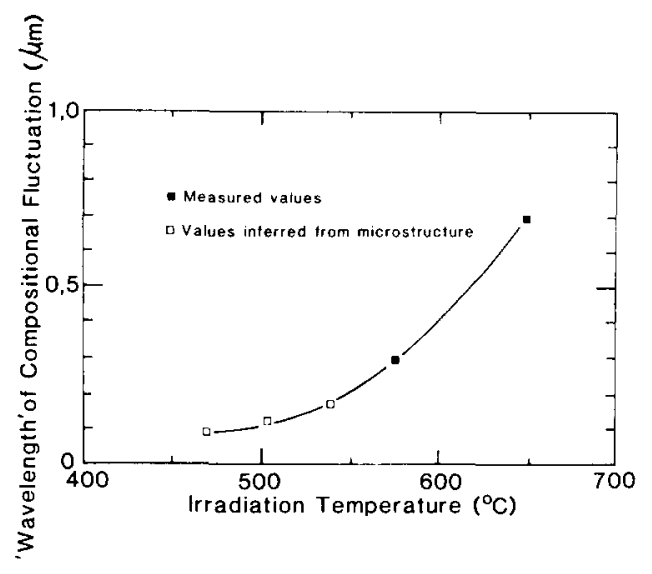

Fig. 49. Variation in the scale of compositional fluctuation with irradiation temperature in the alloy in Fig. 48, irradiated in EBR II. (from ref. 134) 
These transformations are a consequence of extensive irradiation-induced solute redistribution. However, the authors argue [134] that the intragranular solute redistribution does not appear to be associated with point defect sinks, but that segregation may have originally occurred at sinks such as dislocation loops which subsequently grew away or that irradiation-enhanced spinodal decomposition is responsible.

Murphy [164] recently addressed these results based on calculations he performed using the 'random-alloy model' for diffusion in concentrated alloys developed by Manning [165]. His intent was to investigate whether an irradiation-induced instability can produce compositional fluctuations in a concentrated alloy that is thermodynamically stable. He determined that spinodal decomposition was unlikely in thermodynamically stable alloys. Instead he suggested two alternative explanations for the observed compositional instabilities.

It is generally thought that silicon atoms interact strongly with irradiationproduced vacancies and interstitials [166]. These irradiation-induced instabilities caused by dilute concentrations of elements such as silicon produce spatial fluctuations in the concentrations of vacancies and interstitials which in turn can cause fluctuations in the concentrations of the major alloying components. Thus, it is possible that the oscillations in composition in $\mathrm{Fe}-\mathrm{Cr}-\mathrm{Ni}$ alloys arise because of the presence of solute atoms which act as point-defect traps.

An alternative explanation is in the underlying sink structure. Calculation of the wavelengths obtained when only the instabilities in the vacancy-loop population are included [167] show good agreement with the wavelengths of compositional fluctuations observed by Williams et al.[134] at low temperatures. Spatial fluctuations in the density of network dislocations or other point-defect sinks may be able to explain the development of compositional fluctuations at higher temperatures.

A general trend for most of the secondary phases in austenitic and highnickel fcc alloys is that irradiation frequently increases the $\mathrm{Ni}$ and $\mathrm{Si}$ content of thermally existing phases, and radiation-induced phases form with high concentrations of these elements $[130,132,134,135]$. These observations lend strong support for the contention that radiation-induced segregation plays a major role in the development of the phase-microstructure in these alloys. 


\section{B. Metastable phases}

Ion implantation and ion beam mixing are effective methods of forming nonequilibrium or metastable phases in alloys. With this technique, metastable alloys which were previously inaccessible can be readily produced and examined. Since the term "metastable" implies a phase with a free energy higher than that of the stable phase under the prevailing conditions of temperature and pressure, it is natural to look to thermodynamics for an explanation. Experimental results seem to indicate a strong role of thermodynamics in the tendency to form metastable phases. Intermetallic compounds with small ranges of solubility and complex crystal structures are prime candidates for transformation to a metastable phase [168]. Also, the change in the free energy of the solid due to the ion induced defect buildup argues for a thermodynamic explanation [169]. However, not all transformations can be explained on a purely thermodynamic basis.

Hung and Mayer [170] provided a concise summary of the role of kinetics in metastable phase formation. They state that at low temperatures, ion mixing is similar to a quench process where the atom configurations are essentially determined during the relaxation period following the collision events. Because kinetics are restricted, the formation of complex crystalline structures is unlikely and ion mixing will usually result in solid solution, simple cubic structures or amorphous structures. The structure of the metastable system is, however, influenced by the equilibrium nature of the system. Those systems with many intermetallics will tend to form amorphous phases while those with no intermetallic alloys show a tendency to form solid solutions in mixing. At high temperature, atom mobility is significant and equilibrium phases will usually form.

Metastable phases can be formed by ion irradiation, ion implantation and ion beam mixing. Differences in the transformation process between these three techniques can provide insight into the mechanism. For example, in ion irradiation experiments, the main purpose of the radiation is to impart damage to the lattice. However, in ion implantation, the implanted species provides a chemical alteration to the target as well. Ion beam mixing experiments are designed to follow the transformation by rapidly altering the bulk content of the film. Metastable phases formed by irradiation usually occur by one of four types of transformations [171]: 
order $\leftrightarrow->$ disorder

crystal structure A $\rightarrow$ crystal structure B

crystal structure $A \leftrightarrow \rightarrow$ amorphous

crystal structure A $\rightarrow>$ quasicrystalline

Each of these transformations will be reviewed individually, followed by a discussion of the thermodynamic and kinetic factors controlling the transformations.

(i) Order $\longleftrightarrow-\rightarrow$ disorder transformations. Order - disorder transformations can occur in either direction under irradiation, depending upon the composition and structure of the system and the target temperature under irradiation. Schulson [159] and Wilkes et al. [160,161] extensively reviewed the subject of the effects of irradiation on ordering of alloys in the late '70s and early '80s. But the most detailed model of the phenomenon was developed by Banerjee and Urban [162] in which radiation-enhanced ordering is assumed to occur by the thermal motion of vacancies only. The model treats the ordering by vacancy-atom exchange between the sublattices with the activation energy and, therefore, the jump frequency, depending both on the degree of the existing order and on the type of jump. The steady state vacancy concentrations are calculated according to a modification of reaction rate theory [172,173], taking into account the differences in concentrations on the sublattices that can develop in ordered alloys.

At lower temperatures where vacancies are immobile, ion irradiation of ordered alloys often leads to radiation-induced disordering as discussed in section 4.A.v. This occurs very quickly in some systems. For example, the $\gamma^{\prime}$ phase ( $\left.\mathrm{L}_{2}\right), \mathrm{Ni}_{3} \mathrm{Al}$, is extremely unstable under irradiation [174], becoming disordered at a dose of $2 \times 10^{14} \mathrm{i} / \mathrm{cm}^{2}$ [175]. On the other hand, $\mathrm{Fe}_{3} \mathrm{Al}$ (bcc) and FeAl (bcc-B2) undergo only partial disordering after $40 \mathrm{dpa}$ of $2.5 \mathrm{MeV} \mathrm{Ni}{ }^{+}$ irradiation [168]. Hence, factors besides dose play a role in the order-disorder transformation reaction in alloys.

Many compounds undergo chemical disordering prior to amorphization under electron irradiation. Luzzi and Meshii [176] showed that of 32 compounds irradiated, all underwent chemical disordering and 15 amorphized. They concluded that irradiation-induced chemical disordering provided the driving force for amorphization, and cited the difficulty in forming the amorphous structure in pure metals as support for this argument. Until recently [177], there were no observations of chemical disordering prior to amorphization 
during ion irradiation, giving rise to speculation that disordering is not necessary because of the much higher density of damage in ion-induced cascades as compared with electron irradiation. However, as will be shown in section 4.B.iv, disordering prior to amorphization is observed in $\mathrm{Zr}_{3} \mathrm{Al}$ and $\mathrm{FeTi}$, but not in $\mathrm{NiAl}$ which also did not amorphize [177].

(ii) Crystal structure A $\rightarrow$ crystal structure B transformations, Numerous examples exist on the transformation from one crystal structure to another upon ion irradiation. One of the best documented examples is the transformation of a pure metal, nickel, from fcc to hcp under irradiation. This transformation has been found to occur during irradiation with a variety of species including neutrons, chemically inert elements such as $\mathrm{He}$ and $\mathrm{Ar}$, the metalloids $\mathrm{P}$ and As as well as self-irradiation [178]. Observations on P-implanted high purity Ni [179] gave an orientational relationship between the new phase and the fcc matrix similar to that observed for martensitic fcc-->hcp transformations. The transformation in $\mathrm{Ni}$ is thus believed to be martensitic. TEM examination of Sbimplanted $\mathrm{Ni}$ shows hcp particles extending to depths $20 \mathrm{~nm}$ beyond the implanted depth, but dechanneling is present up to $130 \mathrm{~nm}$. This suggests that the defect distribution is playing a role in the structure transformation.

Ion irradiation has also been found to induce phase transformations between bcc and fcc phases in iron-based austenitic alloys [180]. The most prominent example is the fcc to bcc transformation of 304 stainless steel following implantation with $3 \times 10^{16} \mathrm{Fe} / \mathrm{cm}^{2}$ at $160 \mathrm{keV}$. Although this dose amounted to an increase in the $\mathrm{Fe}$ alloy composition by only 1 at\% (67 at\% $\mathrm{Fe}$ nominal), the structure transformed from fcc to bcc. The orientation relationship was neither the Kurdjumov-Sachs nor the Nishijima-Wasserman relationships typically found in ion-irradiated steels [180], but instead obeyed the following relationship:

$$
(100)_{\mathrm{bcc}} \|(100)_{\mathrm{fcc}} \text { and }[010]_{\mathrm{bcc}} \|[011]_{\mathrm{fcc}} \text {. }
$$

Follstaedt [181] suggested that the transformation need not be occurring martensitically, but that the increased defect concentration and hence, diffusivity, may be responsible for the transformation.

It should also be noted that this transformation is inherently different from that of nickel under irradiation. In the present case, the transformation is from the metastable state to the equilibrium structure, whereas in the case of $\mathrm{Ni}$, the transformation is from the equilibrium fcc structure to a metastable hcp structure. Some insight may be gained into the driving force for this type of 
transformation through the experiments of Eridon, Was and Rehn [183]. In these experiments, a target consisting of alternating layers of $\mathrm{Ni}$ and $\mathrm{Al}$ in the atom ratio $3: 1$ was irradiated with $0.5 \mathrm{MeV} \mathrm{Kr}^{+}$ions at 80 and $300 \mathrm{~K}$. Mixing to $1 \mathrm{x}$ $10^{16} \mathrm{Kr} / \mathrm{cm}^{2}$ produced a dual phase structure consisting of disordered $\gamma^{\prime}$ and a metastable hexagonal ideally close-packed phase with the same interatomic distance as the disordered $\gamma^{\prime}$ phase. Thermodynamic modeling of the hcp and disordered $\gamma^{\prime}(\mathrm{fcc})$ phases showed that the heats of transformation, $\Delta \mathrm{H}_{\mathrm{s}-\mathrm{ms}}$ for the two metastable structures were within $\sim 1 \%$ of each other, indicating the lack of a preferred metastable structure, hence the observation of the dual-phase structure.

Several additional examples of crystal structure transformation exist, such as the transformation of the FeV- $\sigma$ phase to a fine-grained, bcc-B2 type structure [168]. Hung et al. $[170,184,185]$ showed that $\mathrm{Al}_{3} \mathrm{Ni}_{2}\left(\mathrm{hP}_{5}\right)$ bombarded with 0.5 $\mathrm{MeV} \mathrm{Xe}+$ to $2 \times 10^{15} \mathrm{~cm}^{-2}$ transformed into the amorphous phase plus AlNi (bcc). The structural transformations in the $\mathrm{Ni}-\mathrm{Al}$ system are summarized in Figs. 50 and 51. Similarly, irradiation of $\mathrm{Pd}_{2} \mathrm{Al}_{3}\left(\mathrm{hP}_{5}\right)$ with $0.5 \mathrm{MeV} \mathrm{Xe}$ ions to a dose of 2 $x 10^{14} / \mathrm{cm}^{2}$ caused decomposition to the $\mathrm{PdAl}(\mathrm{bcc})$ and an amorphous phase [170]. These data indicate that the transformation from one crystal structure to another is readily obtained during ion implantation.

Lilienfield et al. [186] provided an explanation for understanding the transformation of the trigonal (D513) structure (e.g., $\mathrm{Ni}_{2} \mathrm{Al}_{3}, \mathrm{Pd}_{2} \mathrm{Al}_{3}$ ) to the bcc(B2) structure (NiAl, PdAl). The $\mathrm{Ni}_{2} \mathrm{Al}_{3}$ structure can be viewed as being made up of pseudo NiAl cubes, every other one having a vacant $\mathrm{Ni}$ body center site. To construct the $\mathrm{Ni}_{2} \mathrm{Al}_{3}$ structure from the NiAl structure simply requires that every third plane of the $\mathrm{Ni}$ atoms perpendicular to the NiAl [111] direction be replaced by vacancies. This vacancy ordering results in a contraction of the axis parallel to the NiAl [111] direction, which reduces the crystal symmetry from cubic to trigonal.

Since the unit cells of $\mathrm{Pd}_{2} \mathrm{Al}_{3}$ and $\mathrm{Ni}_{2} \mathrm{Al}_{3}$ each have 5 atoms and the unit cells of PdAl and NiAl each have 2 atoms, Lilienfield [186] raises the question of whether stability under ion irradiation depends on the complexity and size of the unit cell. Since atomic species have limited mobility under room temperature irradiation, it is plausible that only simple structures with small unit cells can reorder. This question will be dealt with in more detail in section 4.B.iv on current theories of amorphous phase formation.

Lilienfield et al. [186,187] also produced a metastable phase in the $\mathrm{AlZr}$ system by room temperature ion mixing alternating layers of $\mathrm{Al}$ and $\mathrm{Zr}$ in the composition $\mathrm{Al}_{80} \mathrm{Zr}_{20}$ to form an amorphous structure. The samples were then subjected to either thermal anneals or ion assisted thermal anneals to form the 
metastable phase. This same phase was also formed directly by ion mixing codeposited, amorphous $\mathrm{Al}_{80} \mathrm{Zr}_{20}$ or the multilayered film at elevated temperature. The latter showed that it was possible to form the metastable phase directly by mixing without benefit of a preformed amorphous phase. The resulting phase is an ordered cubic metastable phase that has the same structure as $\mathrm{AuCu}_{3}$, which is an fcc structure with $\mathrm{Au}$ in the corners and $\mathrm{Cu}$ in the faces.
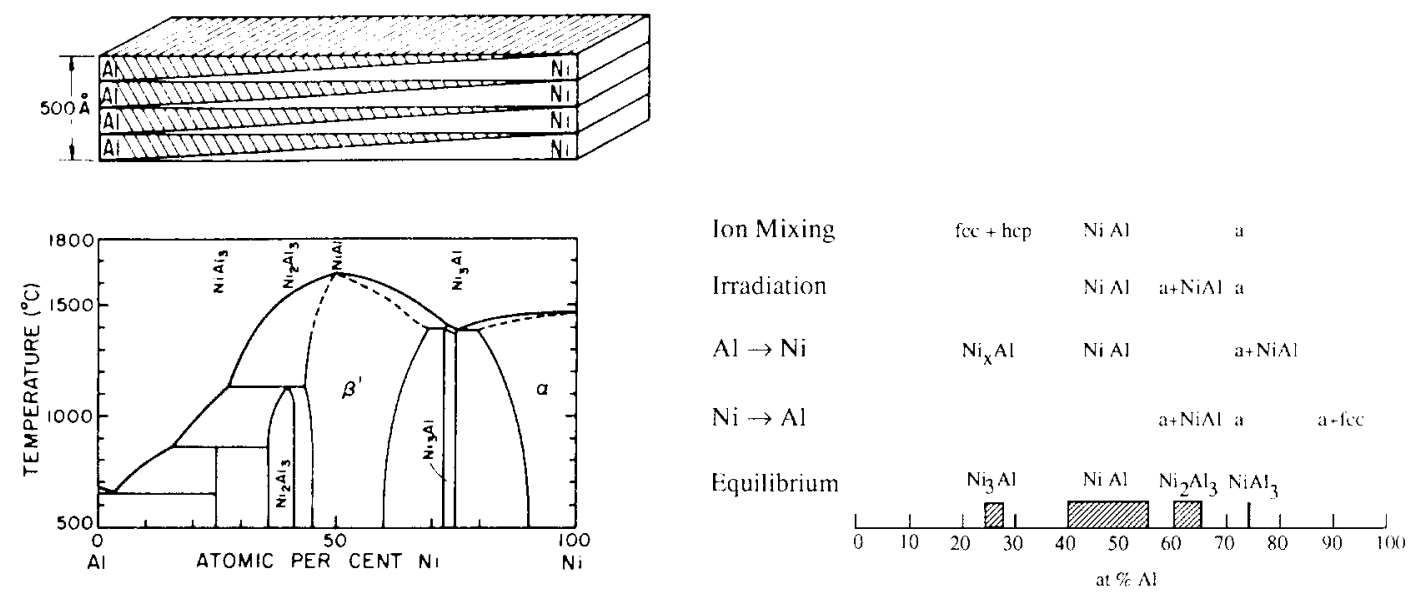

$$
\begin{aligned}
& \text { Anneol: } 350^{\circ} \mathrm{C}-\mathrm{NiAl}_{3}, \mathrm{Ni}_{2} \mathrm{Al}_{3}, \mathrm{NiAl}, \mathrm{Ni}_{3} \mathrm{Al} \\
& \text { Implani: } 500 \mathrm{keV} \times \mathrm{Xe}\left(\mathrm{NiAl}_{3} \longrightarrow\right. \text { omorphous } \\
& 2 \times 10^{15} / \mathrm{cm}^{2} \quad \mathrm{Ni}_{2} \mathrm{Al}_{3} \longrightarrow \mathrm{NiAl} \\
& \text { NiAl }-\mathrm{NiAl} \\
& \mathrm{Ni}_{3} \mathrm{Al} \longrightarrow \text { disordered }
\end{aligned}
$$

Fig. 50. Sample configuration, equilibrium phase diagram of $\mathrm{Ni}-\mathrm{Al}$ and results of multilayered samples preannealed at $350^{\circ} \mathrm{C}$ followed by irradiation with $0.5 \mathrm{MeV} \mathrm{Xe}^{+}$to $2 \mathrm{x}$ Fig. 51. Summary of microstructures in the $\mathrm{Ni}-\mathrm{Al}$ system prepared by various ion beam treatments. (from ref. 171) $10^{15} \mathrm{~cm}^{-2}$. (from ref. 170)

Liu [188] noted that a structurally similar phase of the hcp structure was formed by ion irradiation of multilayered films in five binary (A-B) metal systems (Co-Au, Ti-Au, Co-Mo, Ni-Mo and $\mathrm{Ni}-\mathrm{Nb}$ ) where A refers to the first entry in the alloy designation. The phases were formed in the A-rich multilayered films with overall composition between 65 at $\%$ and 80 at $\%$ A. Further, the spacing of the close-packed planes $\left(\mathrm{d}_{\mathrm{cpp}}\right)$ of all the hcp structures were quite similar. Their formation was attributed to the valence electron effect. For a close-packed hexagonal structure, the minimum number of electron 
states per atom, $\mathrm{n}$, in the Jones' zone can be calculated by the following equation [189],

$$
\mathrm{n}=2-3 / 4(\mathrm{a} / \mathrm{c})^{2}\left[1-1 / 4(\mathrm{a} / \mathrm{c})^{2}\right]
$$

where $a$ and $c$ are the lattice constants of the hcp structure. According to this calculation, the $n$ values of these five phases are almost identical, i.e., $n=1.73$ to 1.74 , which is very close to the value of $1 / 4=1.75$, corresponding to the welldefined Hume-Ropthery $7 / 4$ electron compound. The phases can therefore be considered as metastable electron compounds [190].

It should be noted that from a crystallographic viewpoint, the hcp and fcc structures are similar since both are built by the stacking of close-packed atomic planes differing only in stacking order. Liu therefore states that the structure of the ion-induced phase is always the same as, or similar to the major constituent metal of the alloy.

The formation of metastable phases by ion irradiation of binary alloys exhibiting positive heats of formation has also been demonstrated. Several authors have investigated ion induced phase formation in these systems [191197] and Peiner and Kopitzki [198] characterized ten binary systems. In this study, multilayered samples of ten binary metal systems of different overall compositions were bombarded at $77 \mathrm{~K}$ by $400 \mathrm{keV} \mathrm{Kr}^{+}$ions. All systems had positive values of $\Delta \mathrm{H}_{\mathrm{f}}$, meaning that in thermal equilibrium there is no, or only a limited miscibility of the components of the considered system. However, for the systems $\mathrm{Au}-\mathrm{Rh}, \mathrm{Cu}-\mathrm{Rh}$, and $\mathrm{Cu}-\mathrm{Ir}$, whose components all have fcc structures, a continuous series of single phase metastable fcc solid solutions is produced by ion beam mixing. For the systems Au-Ir, Ag-Ir, and Ag-Rh, they obtained a continuous series of single phase solid solutions upon ion irradiation, and for the other systems an increased solid solubility of one component in the other was achieved. Figure 52 shows the larger one of these solubilities for each system versus $\Delta \mathrm{H}_{f}$ to illustrate the influence of the magnitude of $\Delta \mathrm{H}_{f}$ on the ion beam induced solid solubility. Note that the data points of the systems whose components have the same structures and of systems with components of different structure follow smooth curves. Both curves exhibit a rapid decrease from complete solubility to a solubility below 15 at\% in a range of $\Delta \mathrm{H}_{\mathrm{f}}$ of about $12 \mathrm{~kJ} / \mathrm{mol}$. However, even at large values of $\Delta \mathrm{H}_{\mathrm{f}}$, the solubility still has not dis appeared.

Metastable solid solutions can also form by displacement mixing at temperatures at which radiation-enhanced diffusion is sufficiently slow to maintain the supersaturated solid solution phase. Tsaur et al. [191] showed that 
ion beam mixing of multilayered $\mathrm{Ag}-\mathrm{Cu}$ targets formed a continuous series of metastable solid solutions across the phase diagram. Although the phase diagram of the $\mathrm{Cu}-\mathrm{Ag}$ system is a simple eutectic one with rather small solubilities of $\mathrm{Ag}$ in $\mathrm{Cu}$ and $\mathrm{Cu}$ in $\mathrm{Ag}$, irradiation produced a single phase metastable solid solution with the fcc structure across the entire $\mathrm{Cu}-\mathrm{Ag}$ system. In studies of Au-based systems (Au-Ni, Au-Co), Tsaur et al. [191-193] also found that a single-phase solid solution could be formed over an even wider composition range than that achieved with splat cooling techniques. Since ion beam mixing takes place mainly in the solid state, extended solid solutions can be achieved in nearly immiscible systems such as $\mathrm{Ag}-\mathrm{Ni}$. Even in the binary Au$\mathrm{Fe}$ and $\mathrm{Au}-\mathrm{V}$ systems which have more complex phase diagrams with a large solubility gap and several intermetallic compounds, respectively, and the bcc structure at the Fe- and V- rich terminal solid solutions, Tsaur et al.[193] was able to produce metastable solid solutions across both systems using ion beam mixing. In all cases, the lattice parameters of the solid solutions vary smoothly with composition and show small or moderate deviations from Vegard's law between the appropriate end-members.

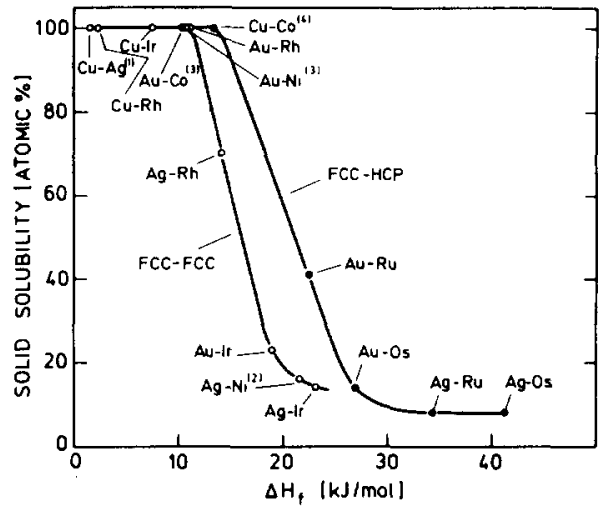

Fig. 52. Irradiation-induced solid solubilities for binary metal systems of positive heats of formation vs $\Delta H_{f}$. (from ref. 198)

(iii) Quasicrystalline phase formation. The most novel metastable phases produced to date are the quasicrystalline phases, produced by ion irradiation of specific Al alloys. These phases show long-range order, but possess forbidden crystalline symmetries such a five- or six-fold symmetry. The phase was discovered by Shectman et al. [199] at the National Bureau of Standards. Quasicrystals have positional order, but are neither periodically nor randomly 
spaced; instead, they are quasiperiodically spaced [200]. This means that, given the position of one unit cell, the positions of the other unit cells are determined according to a predictable but subtile sequence which never quite repeats. Because these structures are highly ordered like crystals but are quasiperiodic instead of periodic, they have been called quasiperiodic crystals, or quasicrystals for short.

Knapp and Follsteaedt [201] and Lilienfield et al. [202] were the first to report the ion-beam-induced formation of the quasicrystalline phase. This work was on the Al-Mn system, but to date, many more binary, ternary and quaternary systems have been shown to form quasicrystals under irradiation [186,187]. In these initial experiments, the quasicrystal phase was formed by irradiating alternating layers of $\mathrm{Al}$ and $\mathrm{Mn}$ in the composition $\mathrm{Al}_{84} \mathrm{Mn} 16$ with $400 \mathrm{keV} \mathrm{Xe}$ ions to doses of $2-10 \times 10^{15} \mathrm{Xe} / \mathrm{cm}^{2}$ at $80^{\circ} \mathrm{C}$. Results showed that the icosahedral phase forms without a separate thermal treatment at or above $80^{\circ} \mathrm{C}$ while the amorphous phase forms at $60^{\circ} \mathrm{C}$. The icosahedron is a regular polyhedra possessing twenty identical triangular faces, thirty edges and twelve vertices. The black pentagons on the surface of a soccer ball are centered on the vertices of an icosahedron. This observed dependence on sample temperature suggests that the icosahedral phase does not form within the dense ion cascade, but rather during subsequent defect evolution. Similar results have been achieved with freestanding Al-Fe multilayered samples [187], indicating that both multilayered and amorphous samples can be transformed to the quasicrystalline phase.

In addition to the temperature window for the formation of quasicrystals in Al-Mn, the composition of the samples has an important effect on quasicrystal formation. Below 80 at $\%$, and above $~ 90$ at $\mathrm{Al}$, quasicrystals could not be formed in any of these systems. Figure 53 summarizes the composition vs temperature data for the three systems. All the data were obtained with implantation of $600 \mathrm{keV} \mathrm{Xe}$ to a fluence of $4 \times 10^{15} / \mathrm{cm}^{2}$. As shown, quasicrystals are formed within a well-defined composition and temperature region.

(iv) Amorphous phase formation. Although the phase space available to an alloy is extremely large, only one point corresponds to an absolute minimum in free energy. This point represents the equilibrium phase. Given sufficient time, at any temperature greater than zero, the system will find that point and settle into the equilibrium phase. Nonetheless, there are generally other minima in the free energy phase space which are of varying depths. These other minima 
correspond to metastable phases. Certain of these phases exhibit compositional short range order (CRSO) very similar to that of the equilibrium phase but different compositional long range order. Similarly, the spatial arrangements of the atoms on a small scale (topological short range order - TSRO) can be very similar to the equilibrium phase but with different long range order (such as fcc vs hcp phases). In general, there may exist many phases with CSRO and TSRO which are nearly identical to the equilibrium phase. Common examples of such metastable phases include glasses and crystalline solids with a slightly different unit cell than the equilibrium.

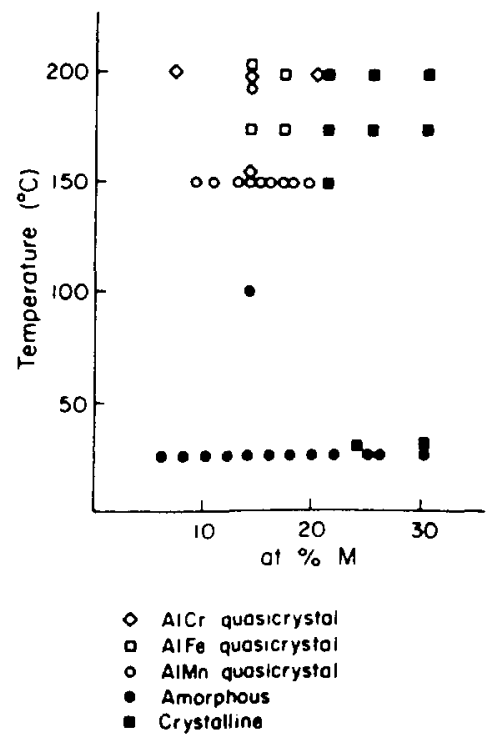

Fig. 53. Temperature versus composition diagram for the Al-Cr, Al-Fe and Al$\mathrm{Mn}$ systems showing the quasicrystalline forming regions. (from ref. 186)

It has been argued that in alloys with large negative heats of formation, disruption of chemical short range order will lead to lattice destabilization and the formation of an amorphous phase [203]. In fact, the data on ion irradiation of intermetallic compounds supports just this sort of conclusion $[168,203]$. Contradicting these observations are results of electron irradiation which shows that complete disordering often precedes the formation of an amorphous phase [176,204]. Furthermore, for intermetallic compounds such as $\mathrm{Zr}_{3} \mathrm{Al}$ or $\mathrm{FeTi}$, electron irradiation disorders but does not amorphize the compounds [206]. Irradiation with light ions produces much the same result as electron irradiation in that the amorphous phase is difficult to form. This suggests that disruption of CSRO is not adequate for lattice destabilization and that another mechanism must be responsible for amorphization such as topological disorder [205-209]. In fact, the self-ion irradiation of $\mathrm{Ni}$ which induces a phase change from stable fcc to 
metastable hcp as discussed in section 4.B.i, clearly has no chemical component and must be a result of the topological disorder introduced into the system by the $\mathrm{Ni}^{+}$beam.

A significant number of metal-metal and metal-metalloid alloys can be rendered amorphous during implantation at sufficiently low temperature. Yet, at present, no general theory has been developed to predict which alloys can be expected to become amorphous. However, a set of empirical rules have been proposed to "correlate" the tendency for a system to amorphize under irradiation with various material parameters. These have been reviewed most recently by Follstaedt [181] and Ziemann [182]. The rules are as follows:

\section{$R<0.59$ (Hägg rule) for interstitial metalloids \\ Negative heat of compound formation}

Simple structures rule

Structural difference rule

Solubility range of compounds/critical defect density

Each of these rules will be treated in individual sections with emphasis on their possible significance in the amorphization mechanism. The various theories proposed to explain ion-induced amorphization are incorporated into the appropriate sections.

\section{(a) Hägg Rule}

The Hägg rule [210] states that if the ratio ( $R$ ) of the metalloid atom radius to the metal atom radius satisfies $R<0.59$, a compound with a simple structure will form in which the metalloid occupies interstitial sites in a metal lattice. If $R>$ 0.59 , a simple embedment of the metalloid atoms in the crystal lattice of metals is not possible. The occurrence of structural rearrangement processes would be necessary, but these cannot take place within the very short lifetime of the thermal spike. Hence, the amorphous structure initiated by irradiation is frozen in. Shown in Fig. 54 [211], the $R=0.59$ lines forms a clear division between the implanted alloys found to be crystalline compounds and those found to be amorphous. Melt quenching studies suggest that amorphous compounds will not be formed for $\mathbf{R}>0.88$, Fig. 54, but Grant et al. [212] show that Ni can be amorphized by $\mathrm{As}, \mathrm{Sb}$ or $\mathrm{Bi}$ implantation for which $\mathrm{R}=0.96$ to 1.18 . Hence, the upper limit on the size ratio is not valid for implantation-induced amorphization. 


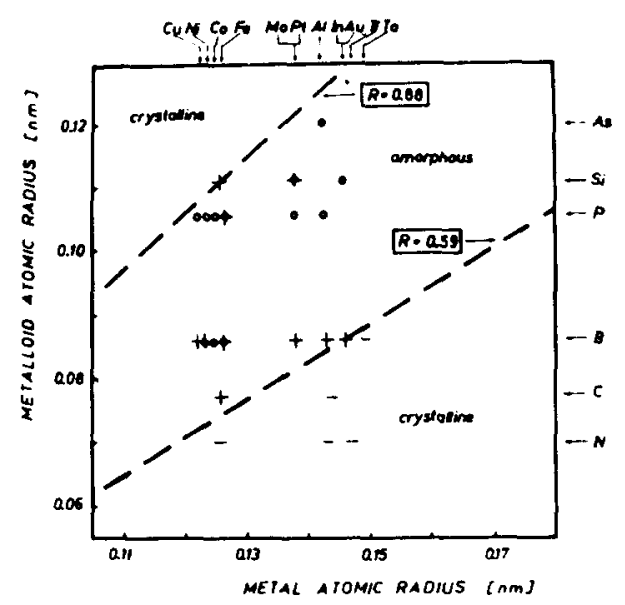

Fig. 54. Summary of the observations of amorphous $(0,+)$ and crystalline (-) alloys in metals implanted with metalloid ions, shown as a function of the two atomic radii. (from ref. 211)

This atomic size, or structural criterion is augmented with a criterion based on electronic considerations. According to Hafner [213], the tendency for glass formation can be correlated with a high negative enthalpy of formation. Miedema et al. [214] argue that the formation enthalpy consists of a negative contribution, $\Delta \phi^{*}$ corresponding to the chemical potential difference and a positive contribution $\Delta n_{\mathrm{w}-\mathrm{s}}$ corresponding to the difference of the electron densities at the boundary of the Wigner-Seitz cell. Using these definitions, the criterion for the formation of amorphous metal-metalloid compound formation by ion implantation becomes [215]

$$
\Delta \phi^{*} \leq 0.75 \Delta \mathrm{n}_{\mathrm{w}-\mathrm{s}}{ }^{1 / 3},
$$

where $\Delta \phi^{*}$ is in volts and $\Delta \mathrm{n}_{\mathrm{w}-\mathrm{s}}$ is in $6 \times 10^{22}$ electrons $/ \mathrm{cm}^{3}$. By this criterion (consisting of a structural and an electronic aspect) Hohmuth et al. [216] claim that it is possible to predict the formation of amorphous metal-metalloid alloys after ion implantation.

Andrew and coworkers [217] have treated the stability of implantationinduced amorphous phases on the basis of the nearly free-electron approach by Nagel and Tauc [218]. They argue that for $Q=2 \mathrm{k}_{f}$, where $Q$ is the wave vector of the first peak in the structure factor and $k_{f}$ is the Fermi wave vector, the Fermi level falls into a minimum of the electronic density of states. In the freeelectron limit the Nagel-Tauc theory reduces to a valence electron concentration 
predicting optimum glass formation for a valence electron concentration $=2$ eV/atom. For a small number of amorphous ion-target systems only, for which data are available [219] this rule is satisfied. Hence, the rule is not universally accepted.

Naguib and Kelly [220] have proposed a model based on the concept of the thermal spike. They argue that the region surrounding the ion track in a solid can be regarded as a small, hot disordered region which is equivalent to a liquid and is surrounded by crystal. Crystallization occurs in an epitaxial regrowth fashion at the liquid-solid interface. But regrowth only occurs if the temperature at the interface is below the solidus melting temperature, $T_{m}$ and above the temperature of crystallization, $T_{c}$. This leads to the criterion that a substance amorphizes if $T_{c} / T_{m}>0.3$, and remains crystalline if $T_{c} / T_{m}<0.3$ [220]. They go on to complement this model with a bond-type criterion which accounts for the nature of the bond, i.e., ionicity in the tendency for a compound to amorphize during irradiation. This criterion states that substances with an ionicity $<0.47$ will amorphize on ion impact [220], where ionicity is defined as

$$
\text { ionicity }=1-\exp \left\{-0.25\left(\mathrm{X}_{\mathrm{A}}-\mathrm{X}_{\mathrm{B}}\right)^{2}\right\} \text {, }
$$

and $X_{A}$ and $X_{B}$ are the electronegativities of atoms $A$ and $B$. According to eqn. (4.10), the bond-type is determined only by the difference $\left(X_{A}-X_{B}\right)$. Although this rule holds for some 52 of 56 non-metallic compounds studied, the rule seems to fail for other systems [219].

\section{(b) Negative heat of compound formation}

Alonso and Simozar [221] noted that a good correlation exists between the heat of formation of a compound and the formation of amorphous phases in metal-metal systems. They found that systems with large negative heats of formation tended to amorphize under irradiation, and constructed a plot of the ratio of the atomic radii and the heat of formation calculated using the Miedema model [222], Fig. 55. According to Alonso and Simozar [221], alloys do not become amorphous if the heat of formation is greater than $+10 \mathrm{~kJ} / \mathrm{mol}$. However, it was demonstrated [193] recently that the amorphous structure can be formed by ion mixing if the heat of formation is higher, e.g., $\mathrm{Cr} / \mathrm{Ag}, \mathrm{Co} / \mathrm{Cu}$, $\mathrm{Fe} / \mathrm{Cu}$ and Co/Au. More recently, Peiner and Kopitzki [198] have shown that specific compositions in the Au-Ir, Au-Ru and Au-Os systems, whose heats of formation range from +19 to $+27 \mathrm{~kJ} / \mathrm{mol}$, can be made amorphous by irradiation. 


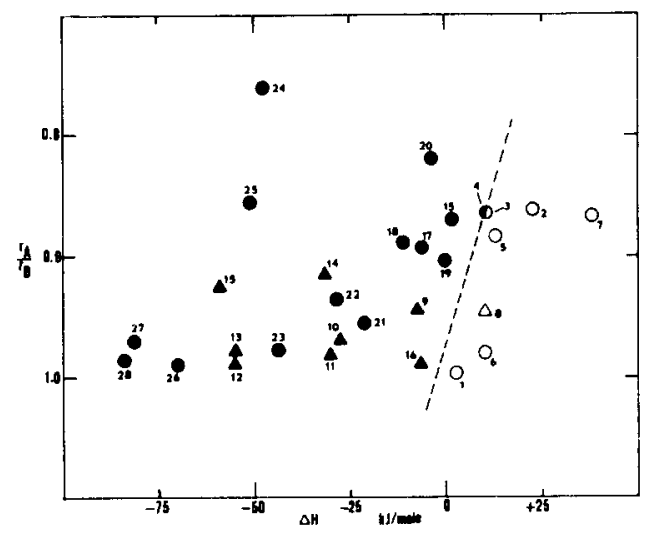

Fig. 55. Ratio of atomic radii versus the heat of formation of an equiatomic compound (o metal-metal, crystalline, - metal-metal amorphous, $\Delta$ metalmetalloid crystalline, $\Delta$ metal-metalloid amorphous). (from ref. 221)

\section{(c) Simple structures rule}

Hung et al. [184] proposed that ion beam mixing will result in the amorphous phase whenever the overall composition is not close to that of a compound with a simple crystalline structure. Conversely, when the overall film composition is close to an equilibrium alloy with a simple structure (fcc, bcc, hcp), the crystalline phase will be formed. This rule was constructed as a result of mixing experiments on the $\mathrm{Al} / \mathrm{Pt}, \mathrm{Al} / \mathrm{Pd}$ and $\mathrm{Al} / \mathrm{Ni}$ systems. It was observed that crystalline phases of simple structures, such as solid solutions or simple cubic, can be formed while amorphous structures are formed with more complex structures. This is explained by the short duration of the relaxation stage following the thermal spike. The authors argue that during the relaxation period, atoms attempt to rearrange themselves. If the relaxation time is sufficient for precipitates to nucleate, crystalline phase formation may be achieved. The time required for nucleation is strongly influenced by the temperature, the crystalline structure of nulcei and the composition of the films which have been homogenized with thermal treatments or ion beams. If the overall composition is not close to a simple crystal structure in the equilibrium phase diagram and there is not a strong chemical driving force (as well as mobility) to promote significant atomic motion, crystalline phase formation may be inhibited. Recall that Alonso and Simozar [221] concluded that the occurrence of an amorphous alloy produced by ion mixing is strongly linked to the existence 
of an equilibrium compound, and hence, with a negative heat of formation. Although this works for many compounds, extensive experimentation has revealed that many alloys with simple equilibrium crystal structures (eg., $\mathrm{NiAl}$, FeTi, NiTi, CuZn, etc.) become amorphous under irradiation.

\section{(d) Structural difference rule}

This rule was formulated by Liu $[223,224]$ in 1983 and states the sufficient conditions for producing amorphous alloy films by ion mixing of multiple alternate metal layers: (1) the constituent metals have different structures, and (2) the composition after uniform mixing lies within the two-phase region of the equilibrium phase diagram. This rule predicts amorphous alloy formation irrespective of the atomic size and electronegativity properties of the constituents, as long as the constituents have different crystal structures. Although Liu cites several examples of systems for which this rule is obeyed, there are a large number for which it is not obeyed. For example, FeAl (bcc-fcc) does not amorphize after ion doses up to $40 \mathrm{dpa}$ [168]. Similarly, Ni $3 \mathrm{Ti}$ (fcc-hcp) does not go amorphous. In fact, the converse of this rule often is obeyed. That is, constituents of the same crystal structure have been found to amorphize: $\mathrm{NiAl}_{3}$ (fcc), $\mathrm{PdAl}_{3}$ (fcc), several $\mathrm{Pt}-\mathrm{Al}$ (fcc) alloys, and $\mathrm{Al}_{2} \mathrm{Au}$ (fcc) are a few examples. Further, it should be noted that the composition appears to play a significant role, since in the $\mathrm{Ni}-\mathrm{Ti}, \mathrm{Ni}-\mathrm{Al}$ and $\mathrm{Pd}-\mathrm{Al}$ systems, only certain alloy compositions amorphize under irradiation. Hence, although it provides a general guideline, the structural difference rule is not universally obeyed. As a result, Liu [190] has concluded that the rule is a sufficient, but not a necessary condition.

Liu [190] proposed an extended structural difference rule based on the use of the equilibrium phase diagram for the binary alloy under consideration. The rule states that if the overall composition is in the two-phase region of the phase diagram, an amorphous alloy will most likely form. If the overall composition is in or near the single-phase region of the phase diagram and the structure of this phase is not simple, an amorphous alloy is likely to be formed. Finally, if the overall composition is in or near the single-phase region of the phase diagram and the structure of this phase is simple, a crystalline phase is formed. This model can be understood with reference to Figs. $56 \mathrm{a}$ and b, which are a representative phase diagram and the corresponding free energy- composition diagram. Regions 1,2 and 3 refer to the single phase region where the phase is a solid solution with a simple crystal structure (fcc, bcc, hcp), a two-phase region, and a single phase region, typically an intermetallic compound which exists in a 
narrow composition range and frequently possessing a complicated structure, respectively. In the free energy diagram, points $x, y$ and $z$ refer to the equilibrium states of the alloys having the compositions 1,2 and 3 respectively. Points $\mathrm{X}, \mathrm{Y}$ and $\mathrm{Z}$ refer to the free energy of the random mixtures immediately after mixing (prompt process). Along the path toward equilibrium, metastable states may be encountered which have free energies greater than equilibrium but less than the random mixture. The curve labeled "amorph." refers to the amorphous states.
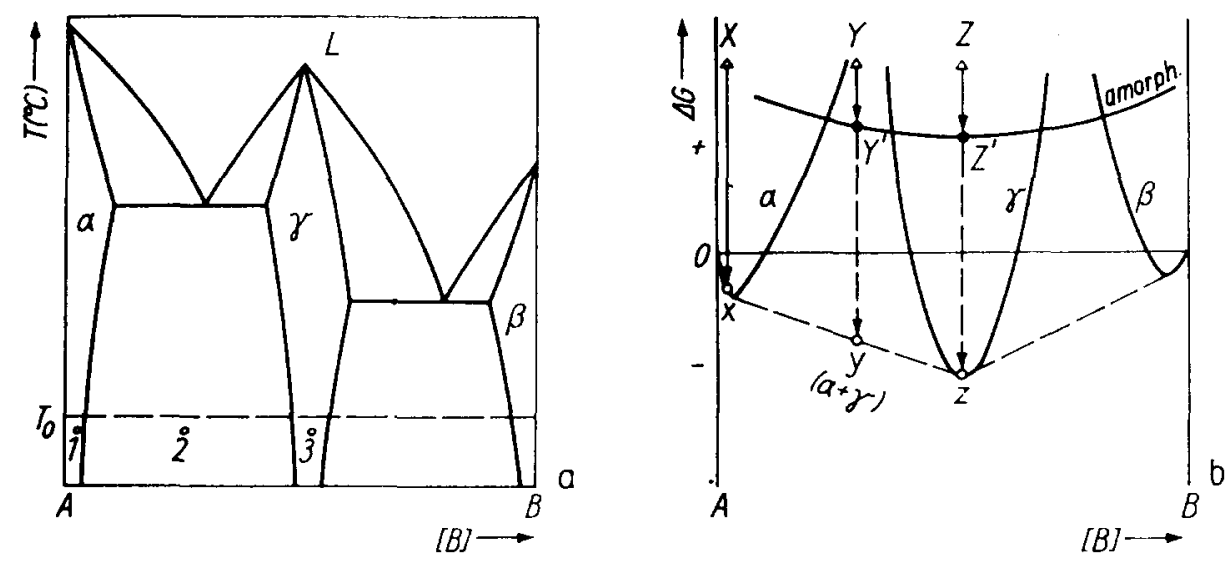

Fig. 56. Representative phase diagram (a), and the corresponding free energycomposition diagram (b) ( $\Delta$ excited state, $\bullet$ amorphous state, o equilibrium state) for a binary alloy. (from ref. 188)

In region 2 , the transition from $\mathrm{Y}-\rightarrow \mathrm{y}$ requires an adjustment in composition involving large-scale atomic movement via diffusion, which will be difficult due to the short duration of the thermal spike and the low temperatures at which the mixing is conducted. However, the $\mathrm{Y}-\rightarrow \mathrm{Y}^{\prime}$ ( $\mathrm{Y}^{\prime}$ is amorphous) is polymorphic (diffusionless transformation) and will be favored.

In region 3, both transitions, $\mathrm{Z}-->\mathrm{z}$ and $\mathrm{Z}-->\mathrm{Z}^{\prime}$ are both polymorphic, but the more complicated $\gamma$ phase requires greater atom mobility and thus, its formation will be kinetically limited. The amorphous phase will be favored.

In region $1, X-\rightarrow x$ is polymorphic, and since the terminal solid solution always possesses a simple crystal structure with high growth kinetics and little atomic movement, the transition can be completed within the relaxation period of the thermal spike (10 ps). 
Figure 57 is an ion-induced phase diagram constructed from the equilibrium phase diagram, and the free energy-composition diagrams of Fig. 56. The appearance of the metastable $M X$ phase can be justified by noting that the free energy curve of this phase should be similar to that of a compound, e.g., the $\gamma^{\prime}$ phase, and the transition from the random mixture to the MX state is therefore polymorphic. Second the MX phase is of a simple structure, ie., an enlarged hcp structure, therefore, the crystallization process can be completed during relaxation.

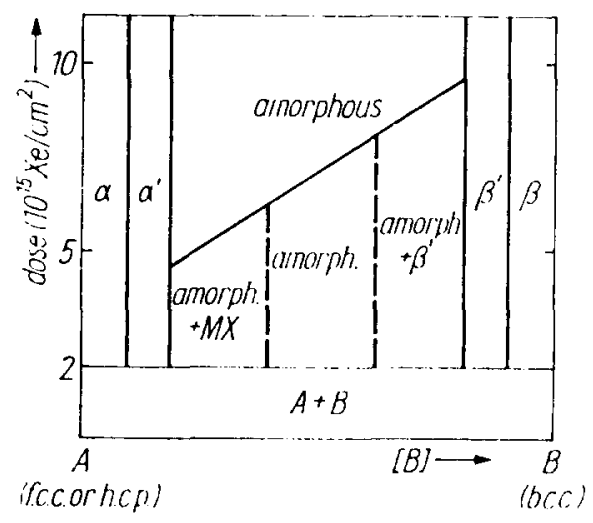

Fig. 57. Ion-induced phase diagram. Extended (supersaturated) solid solutions $\alpha^{\prime}$ and $\beta^{\prime}$ are obtained from both sides of the phase diagram. As the composition approaches the middle of the phase diagram from the side A, a mixture of amorphous phase and MX phase (hcp) is formed at intermediate dose, while a mixture of an amorphous phase and $\beta^{\prime}$ phase is obtained approaching from the B side. At high dose, a uniform amorphous phase is formed. (from ref. 188)

Liu presents extensive data to support these observations in each region of the phase diagram. The rule succeeds, not only in explaining the amorphization of a large number of metal combinations with different lattice structures, but also with the same lattice structure.

A set of rules related to the structural difference rule has been proposed by Johnson et al. [30]. In their treatment, they make use of a binary constitution diagram of an A-B alloy, Fig. 58, a schematic free energy diagram for this alloy, Fig. 59, and the corresponding polymorphic phase diagram, Fig. 60. Note that the $T_{o}$ curves are obtained from the crossing of the solid free energy curve with that of the liquid (or amorphous) curve at a given temperature. The $\mathrm{T}_{0 \alpha}$ line defines the thermodynamic composition limits of the $\alpha$-solution. When the concentration profile induced by mixing falls locally outside these limits, the $\alpha$ - 
solution is superheated with respect to the liquid (amorphous) phase and is not stable. Since melting is a local phenomenon not involving long range diffusion, and since solids are not observed to withstand extensive superheating, it follows that observation of an $\alpha$-phase outside these composition limits represents an unstable state. Such a state will likely melt or amorphize before thermal spike evolution is complete.

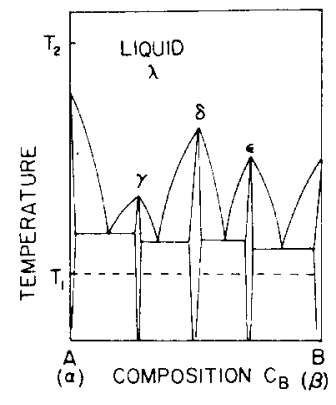

Fig. 58. Binary constitution diagram of an A-B alloy. (from ref. 30)

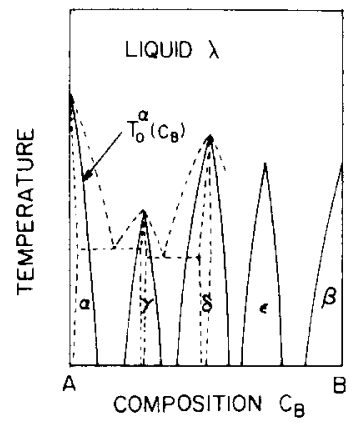

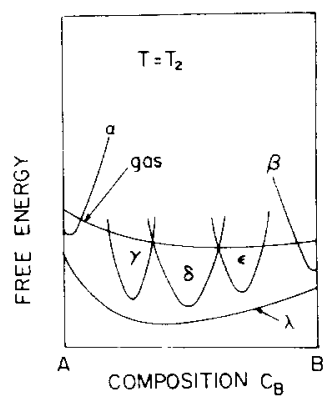

Fig. 59. Schematic free energy diagram for the alloy of Fig. 58 at temperature $T_{1}$, crossing of $\alpha$ and $\lambda$ curves defining the $T_{0} \alpha$ curve. (from ref. 30)

Fig. 60. Polymorphic phase diagram corresponding to the equilibrium phase diagram of Fig. 58. Dashed lines show part of the original equilibrium diagram. Solid lines are representative $T_{0}$-lines of each phase. The $\mathrm{T}_{0}$-lines define regions of polymorphic solid formation from the liquid state. Regions outside correspond to liquid or amorphous (polymorphic) states. (from ref. 30)

This leads to the first fundamental rule for solid phase formation. Johnson et al. [30] assert that terminal solid solutions $\alpha$ and $\beta$ can be formed up to the limits of the $T_{o \alpha}$ and $T_{o \beta}$ curves (i.e. within the polymorphic phase diagram limits of $\alpha$ and $\beta$ ). Solutions formed outside these limits are superheated and unstable against amorphization or melting. Secondly, intermetallic compounds with broad 
equilibrium homogeneity ranges (wide polymorphic limits in Fig. 60), and low energy interfaces with $\alpha$-solution or $\beta$-solution phases, may form in the prompt cascade provided that their respective growth kinetics allow high growth velocities. Compounds with narrow homogeneity range and complex chemically ordered unit cells should not form. Finally, amorphous phases are expected whenever the ion induced composition profile $C_{B}(z)$ lies outside the polymorphic limits of crystalline phases. Amorphization may occur in addition when polymorphic limits permit compound formation but kinetics of compound formation or growth are slow.

Martin [225] proposed a theory for amorphization which is similar in nature to that of Johnson. In this model, he adds the ballistic radiation recoil resolution displacement jumps to thermally activated jumps to obtain an irradiationaltered diffusion equation. The practical effect of irradiation is then to cause the system to assume the configuration at temperature $T$ that it would have at a temperature $T^{\prime}$ outside irradiation: $T^{\prime}=T\left(1+D_{B}^{\prime} / D^{\prime}\right)$, where $D_{B}{ }^{\prime}$ is the ballistic diffusion coefficient, due to displacements and $D^{\prime}$ is the interdiffusion coefficient in the absence of ballistic effects, Fig. 61. The theory predicts that irradiation of an equilibrium alloy of two solid phases at temperature $T$ could raise the "effective," temperature to $T_{1}{ }^{\prime}$, or, under sufficiently intense irradiation to $T_{2}$ '. At $\mathrm{T}_{1}{ }^{\prime}$, the irradiated alloy would at steady state, be composed of amorphous and crystalline phases of different compositions. Irradiation intense enough to raise the effective temperature to $T_{2}{ }^{\prime}$ would produce a uniform amorphous alloy. The predictions of this theory have yet to be tested.

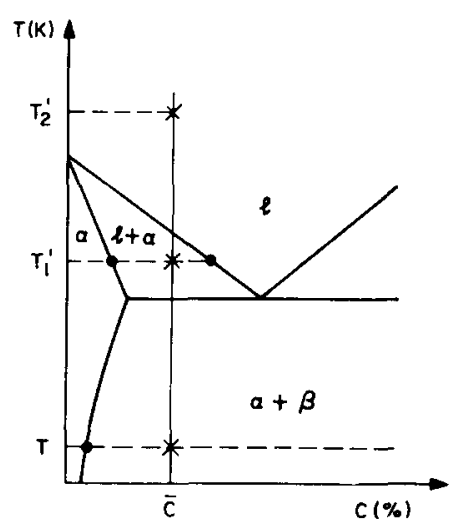

Fig. 61. Possible configuration of an equilibrium, two-phase alloy under irradiation at temperature $T$. Irradiation to an effective temperature $T_{1}{ }^{\prime}$ gives amorphous and crystalline phases of different composition, while more intense irradiation to an effective temperature $T_{2}{ }^{\prime}$ gives a single uniform amorphous phase. (from ref. 225) 


\section{(e) Solubility range of compounds / critical defect density}

Brimhall [168] noted that the tendency toward amorphization of the intermetallic compounds by irradiation correlated reasonably well with the degree of solubility within the phases. That is, alloys with limited solubility or a narrow compositional range show greater tendency for amorphous transformation. This correlation is also consistent with the concept that a critical energy or defect density must be created before the amorphous transformation can occur. If the total free energy of the defect crystalline state becomes greater than that of the amorphous state, a spontaneous transformation should occur. Associated with this critical free energy is a critical defect concentration. If this critical defect concentration can be reached under irradiation, then the crystal should relax into the lower free energy (amorphous) state. This critical defect concentration has been estimated at 0.02 for silicon and germanium [226].

The link between defect density and degree of solubility can be seen by referring to the free energy diagram in Fig. 62. Compounds with no or limited compositional range will undergo a greater increase in free energy than those with wide compositional range. The greater increase in free energy is due to the inability of the compound to exist in equilibrium outside the designated compound. This is manifest in the narrow and steeply rising free energy vs composition curves. For ordered phases (intermetallics) the increase is not only due to point defects, but antisite defects in regions of localized nonstoichiometry. This proposed solubility rule is very consistent with Johnson's thermodynamic analysis [30]. Note that for $\mathrm{NiAl}_{3}$, only a slight deviation from stoichiometry is needed to result in a very large rise in the free energy. Therefore, the critical defect density would be low for $\mathrm{NiAl}_{3}$ as compared to NiAl.

Antisite defects may play a critical role in the amorphization process since calculations have shown that the critical defect density may be difficult to reach accounting for only point defects [227]. However, since the defect concentration is strongly dependent on atom mobility and this is largely unknown in intermetallic compounds, accurate estimates of defect concentration are difficult to determine. Further, the critical defect density will be strongly temperature dependent with higher concentrations required at higher temperatures.

Pedraza [205-207] has extended this theory to include the existence of a defect complex. Because simple point defects do not normally reach the levels needed for amorphization during irradiation, she postulated a defect complex consisting of a vacancy and an interstitial. The likely site for such an interstitial is one where the chemical nature of the geometric neighbors allows a situation resembling that in the normal ordered lattice. The role of the vacancy is to allow for partial volume relaxation. The interstitial will also have a higher tendency 
for remaining in this site if there is a vacancy nearby. Thus the formation of the complex constitutes a mechanism for relaxing local stresses while creating a center of short-range order and a focus of topological disorder under irradiation. This is what is needed for promoting a disordered structure under irradiation.
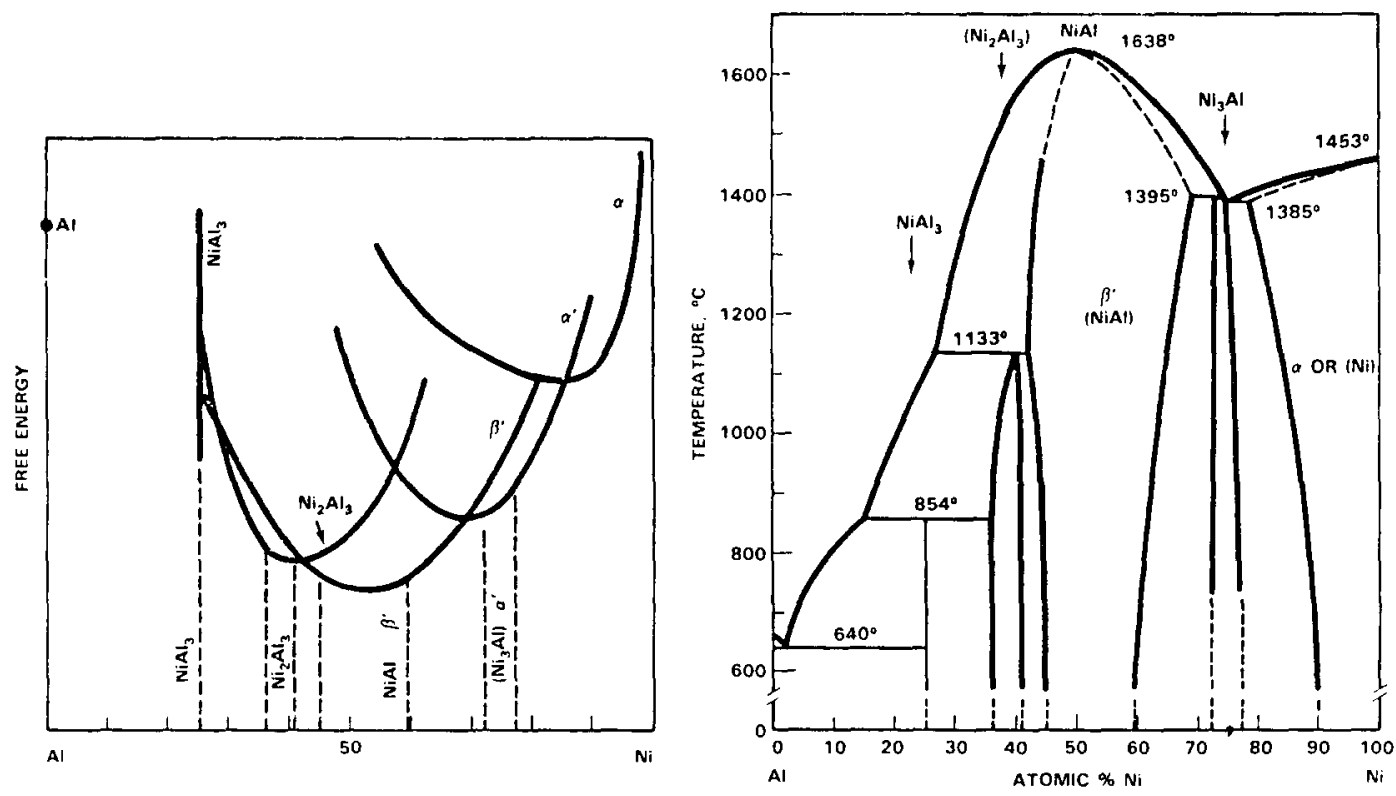

Fig. 62. NiAl phase diagram with hypothetical free energy diagram at the irradiation temperature. (from ref. 168)

Further support to the critical defect density idea is provided by the observation that compounds that go amorphous during irradiation do not show evidence of prior dislocation loop formation [228,229]. Although it has been assumed that the attainment of a critical defect concentration will cause an amorphous transformation, the high free energy associated with the point defects can also be relieved through the formation of dislocation structure. This structure evolves from the initial collapse of interstitial or vacancy clusters into small loops. However, either a material goes directly to the amorphous state or forms dislocation loops.

Since greater mobility is required for the formation of dislocation loops, and the maximum defect concentration associated with the nucleation of loops in 
irradiated metals is $\sim 10^{-4}$ [227], almost two orders of magnitude less than that for amorphization, this indicates that defect mobility is a key factor in the amorphization process. This observation is consistent with the ease of amorphization in intermetallics where defect mobility is low, and the preferential formation of dislocation loops in pure metals and solid solution alloys where the mobility is high.

Brimhall [168] explains some of the many exceptions to this rule (e.g., the $\operatorname{ReTa} \chi$ phase which has a wide compositional range yet becomes amorphous upon ion bombardment) by a low defect mobility at the irradiation temperature. Similarly, he claims that high solubility in nickel explains why compounds on the nickel rich side of the $\mathrm{Ni}-\mathrm{Al}$ and $\mathrm{Ni}-\mathrm{Ti}$ phase diagrams remain crystalline while the low solubility of $\mathrm{Ni}$ or $\mathrm{Ti}$ in $\mathrm{Al}$ explain why the aluminum rich compounds readily amorphize. Hence, when an element or phase shows very limited solubility for another element or phase, the amorphous transformation is more likely to occur during irradiation. In these compounds with limited compositional range, the basic compositional unit will try to maintain itself, resulting in a high degree of short-range order (SRO) in the amorphous phase. Since only short-range motion and relaxation occurs at these low irradiation temperatures, the SRO regions are highly misoriented with respect to each other. Because the low defect mobility does not permit long-range ordering, the amorphous structure forms.

Eridon et al. [183] argue that such short range order exists in the amorphous phase of composition $\mathrm{NiAl}_{3}$. Calculations indicate that either a disordered crystalline (orthorhombic) structure or a disordered amorphous (no short-range order) structure will have a significantly higher free energy than an ordered amorphous (short-range order) structure.

Brimhall [168] points out that the compositional range or extent of solubility in a phase is not a fundamental physical parameter but is, in fact determined by the combination of other factors such as bonding, atom size, and electronic structure.

\section{(f) Spike and temperature effects}

From the preceding discussion, it is evident that many of the proposed amorphization mechanisms rely on the existence of a cascade. Brimhall et al. [229] irradiated $\mathrm{NiTi}$ with $2.5 \mathrm{MeV} \mathrm{Ni}^{+}$and $9 \mathrm{MeV} \mathrm{Ta}^{+}$and found that the amorphous volume fraction as a function of dose in dpa exhibited supralinear behavior, Fig. 63, which is consistent with a cascade overlap model for the formation of amorphous zones. Simonen [230] has modeled the volume fraction 
of amorphous phase as a function of dose using the cascade model as well as the point defect model and can fit the experimental data with either. Thus, both models can be made to be consistent with the observed results. Nevertheless, experiments on $\mathrm{Ga}$ and $\mathrm{Al}_{2} \mathrm{Au}$ show that in some cases, amorphization can occur in the absence of cascade formation.

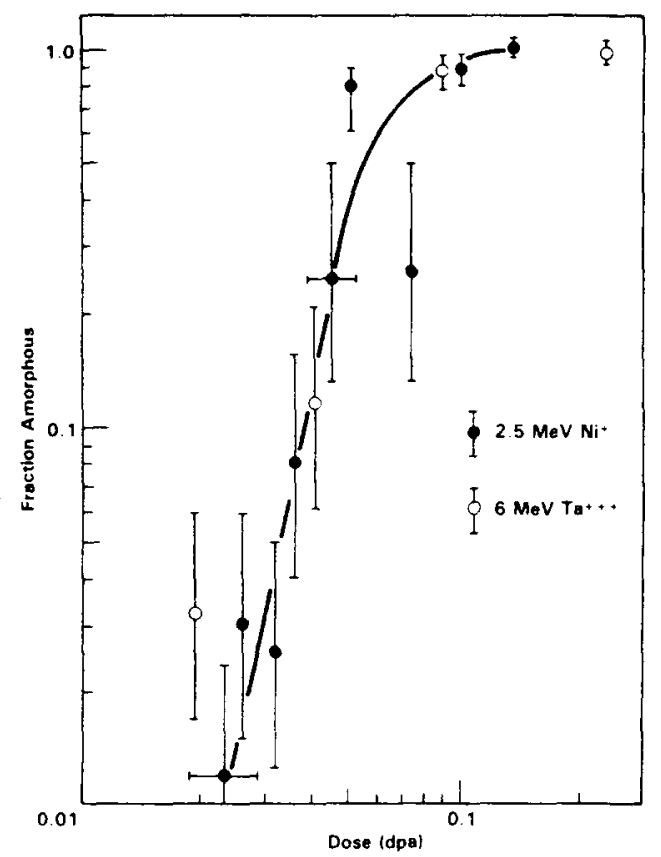

Fig. 63. Amorphous volume fraction as a function of dose in dpa for NiTi bombarded with either $2.5 \mathrm{MeV} \mathrm{Ni}{ }^{+}$ions or $6 \mathrm{MeV} \mathrm{Ta}^{++}$ions. (from ref. 229)

Results of quench-condensation experiments indicate that gallium may be amorphized by ion irradiation. Goerlach et al. [231] conducted experiments designed to determine if pure, elemental gallium can be amorphized by irradiation and if so, whether cascades are necessary. Crystalline $\alpha$-gallium was bombarded with $275 \mathrm{keV}$ argon ions and $200 \mathrm{keV} \mathrm{He}$ ions at $\mathrm{T}<10 \mathrm{~K}$. Results indicated that $\alpha-\mathrm{Ga}$ was transformed to the amorphous phase by Ar irradiation at very small fluences $\left(2 \times 10^{14} \mathrm{~cm}^{-2}\right)$. However, He irradiation failed to produce the amorphous phase even after the same deposited energy. This seems to support the idea that cascades are necessary for the formation of the amorphous phase in this element. The fact that a pure element was amorphized brings into question many of the rules just discussed for amorphous phase formation. 
Another significant set of experiments involved the irradiation of $\mathrm{Al}_{2} \mathrm{Au}$. Since both are of the same crystal structure, the structural difference rule would predict that amorphous phase formation is not possible. However, a rule which states that whenever a system can be forced into the amorphous state by vapor quenching, then ion irradiation will also result in the amorphous phase, does predict amorphization based on vapor quenching experiments by Folberth [232]. Experimentally [233] after irradiation of crystalline $\mathrm{Al}_{2} \mathrm{Au}$ at $\mathrm{T}<10 \mathrm{~K}$ with 250 $\mathrm{keV} \mathrm{Ar}$, an amorphous phase was formed. Amorphization also occurred after irradiation with $200 \mathrm{keV} \mathrm{He}$ at the same temperature. At 80K, irradiation with Ar produced the amorphous phase, but irradiation with $\mathrm{He}$ did not. These results lead to the conclusion that at very low temperatures, spikes are not necessary to amorphize a binary system which is known to become amorphous by vapor quenching. However, at higher temperatures, the high energy density of cascades governs the amorphization process.

\section{(g) Shear instability-driven amorphization}

Okamoto et al. [177,234] have conducted experiments to measure the lattice dilation and shear elastic constant as a function of the degree of long-range order during room temperature irradiation of several intermetallic compounds. In these experiments, $\mathrm{ZrAl}_{3}, \mathrm{FeTi}$ and $\mathrm{NiAl}$ were irradiated with $\mathrm{MeV} \mathrm{Kr}$ ions at room temperature. The lattice parameter and the degree of long-range order was measured in TEM and the shear modulus was measured using Brillouin scattering spectroscopy. Results showed that for $\mathrm{ZrAl}_{3}$ and $\mathrm{FeTi}$, a large lattice dilation and shear modulus softening were observed during chemical disordering, which was followed by amorphization, Fig. 64. The decrease in the shear modulus in $\mathrm{ZrAl}_{3}$ and $\mathrm{FeTi}$ was $50 \%$ and $40 \%$, respectively. NiAl did not become amorphous and showed a drop in shear modulus by only $10 \%$. The lattice dilation in $\mathrm{NiAl}$ was also smaller than in the other two compounds. The observed linear relationship between the increase in lattice parameter and decrease in shear modulus during irradiation is analogous to what occurs during heating to melting [235,236]. Lattice dilation is observed during heating of most materials, and it is accompanied by a decrease in elastic moduli. The strong correlation observed between chemical disordering, lattice dilation, shear modulus softening, and amorphization suggest that solid-state amorphization is triggered by an elastic shear instability driven by lattice dilation and chemical disordering. In fact, $\mathrm{Zr}_{3} \mathrm{Al}$ was amorphized by hydrogen charging without any discernible chemical disordering [237]. The total lattice dilation at which the 
(a)

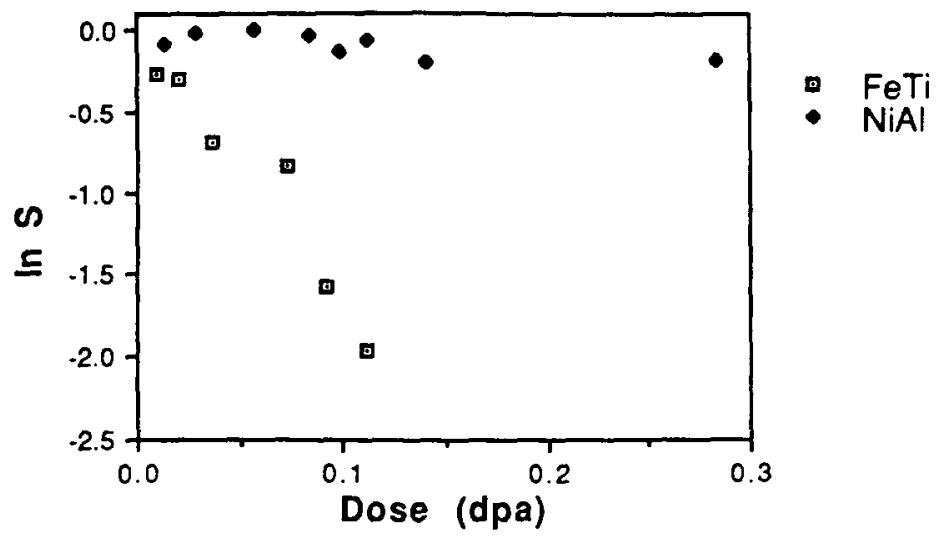

(b)

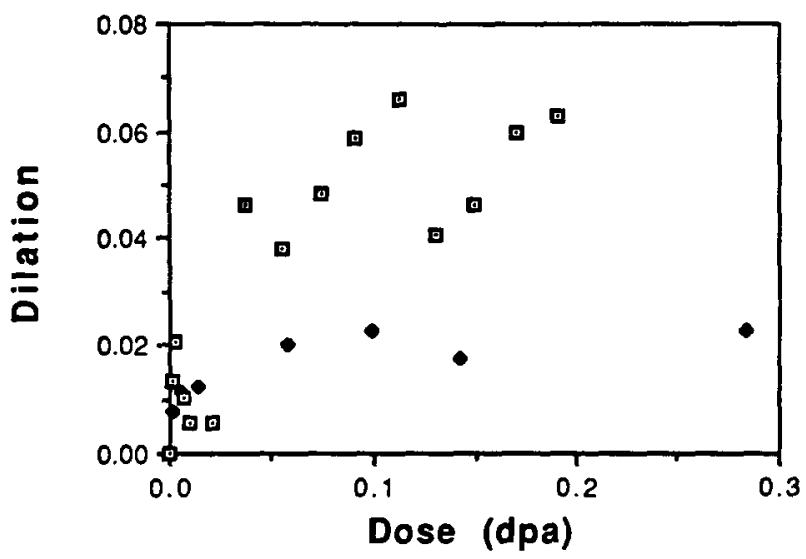

(c)

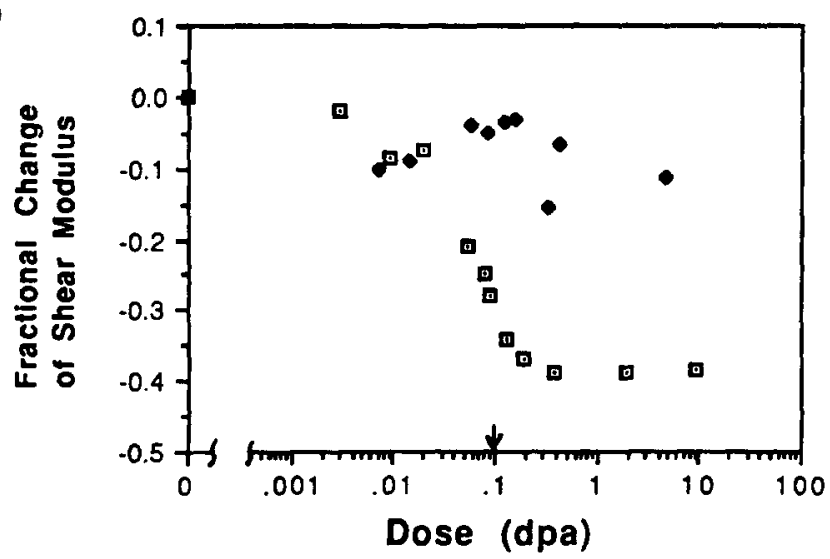

Fig. 64. Variation of the measured parameters of FeAl and NiAl as a function of irradiation dose; (a) chemical long-range order parameter, (b) the lattice dilation, and (c) shear modulus. (from ref. 177) 
onset of amorphization was observed by hydrogen charging was the same as in the case of $\mathrm{Kr}^{+}$irradiation-induced amorphization.

\section{(h) Other theories}

Ossi [203,238] has proposed a model he terms the segregation charge transfer (SCT) model in which he calculates the electronic energy change associated with segregation occurring in the thermalization stage of the cascade and relates this quantity to the surface energy difference between the segregated and original alloy and hence, the potential for forming an amorphous structure. The SCT model accounts for the development of dense well-developed cascades in targets undergoing bombardment by heavy ions. Cascades are assumed to evolve with two separate time scales; during the prompt regime the spike volume may be thought of as a region encompassing a few thousand atoms in extremely violent atomic agitation. When the locally deposited energy is insufficient to displace recoils further from their sites, delayed events dominate.

Atomic rearrangements which occur within the spike volume involve selective migration of one of the atomic species to the spike-lattice interface, which thereby becomes enriched in that component. The violence of cascade mixing is not able to compensate for the composition alteration because the composition peak develops in the neighborhood of impacting ions following the cascade maximum, i.e., during the final fast thermalization stage of cascade atoms. The compositional change at the spike surface induces a local variation in spatial electronic density, subsequently relaxing towards a bulk equilibrium value.

For an A-B alloy, the atom-atom interaction at the surface is represented by an electronic elementary charge transfer process between two atoms, one atom of the surface-enriched species $B$ and one atom of the $A$ type. Such an interaction is governed by the condition that the segregating element behaves in an electron acceptor way, thus giving

$$
\begin{aligned}
& A\left(-1 e^{-}\right) \rightarrow C_{e f f} \\
& B\left(+1 e^{-}\right) \rightarrow D_{e f f}
\end{aligned}
$$

where the C-D effective atom couple is considered as a microalloy cluster in which $D$ segregates with respect to $C$, corresponding to the original alloy in which it is assumed that $B$ segregates with respect to $A$. The electronic energy change $\Delta \mathrm{E}$, is calculated using electron energies for pure elements taken to be isolated. Segregation in both A-B and C-D alloys is studied using Miedema's parameters, 
$\phi^{*}$ and $n_{w-s} s^{1 / 3}$. Figure 65 plots $\Delta\left(\Delta \phi^{*}\right)$, the surface energy difference between alloying elements, against $\Delta\left(\Delta n_{w-s} 1 / 3\right)$, the difference in electron densities at the boundary of the Wigner-Seitz cell, for A-B to C-D alloy transitions in amorphous and crystalline systems. The full line separating the two regimes divides the amorphous alloys, those with lower segregation strength in C-D alloys than in A$B$ alloys (right-hand side) from crystalline alloys (left-hand side). The dashed line separates regions of solute segregation from those of solvent segregation. For amorphous alloys, positive $\Delta\left(\Delta \phi^{*}\right)$ and $\Delta\left(\Delta \mathrm{n}_{\mathrm{w}-\mathrm{s}}{ }^{1 / 3}\right)$ values correspond to solute segregation, while negative values are found when the solvent segregates. The opposite holds for crystalline alloys. Figures $66 \mathrm{a}$ and b show $\Delta\left(\Delta \phi^{*}\right)$ vs $\Delta \mathrm{E}$ for the two classes of systems. Note that a linear correlation exists between the two quantities for both systems.

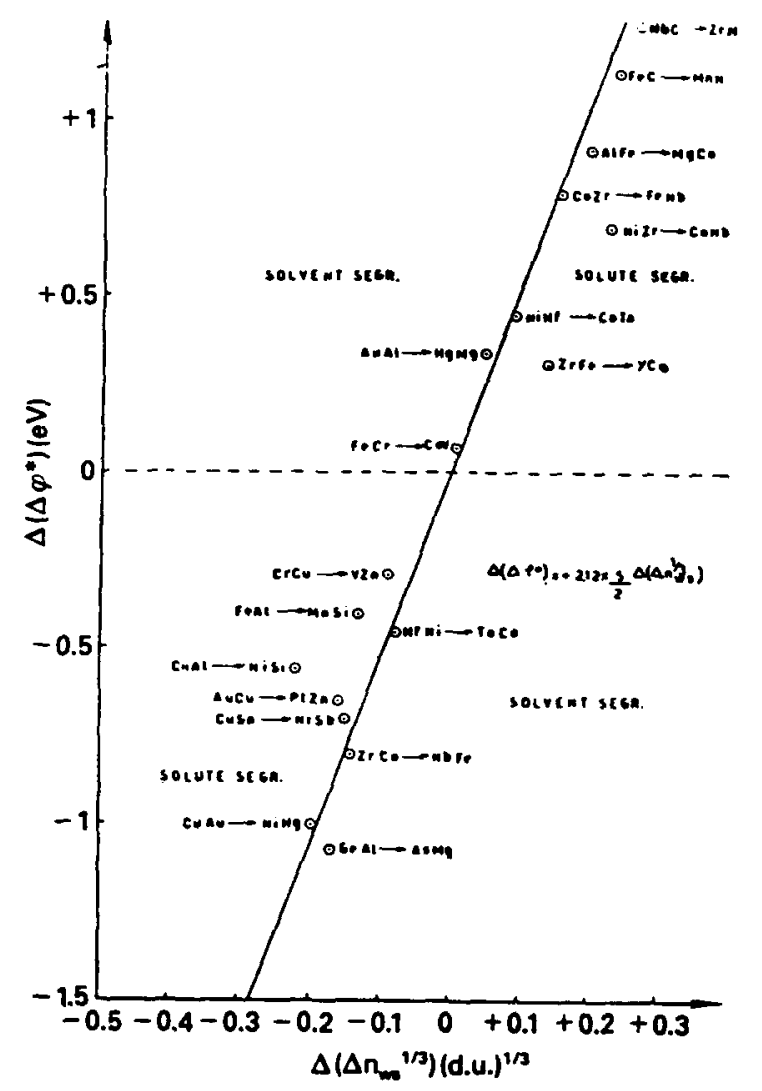

Fig. 65. Calculated $\Delta\left(\Delta \phi^{*}\right)$ vs $\Delta\left(\Delta \mathrm{n}_{\left.\mathrm{w}-\mathrm{s}^{1 / 3}\right)}\right.$ relation for both amorphous and crystalline systems. (from ref. 238) 

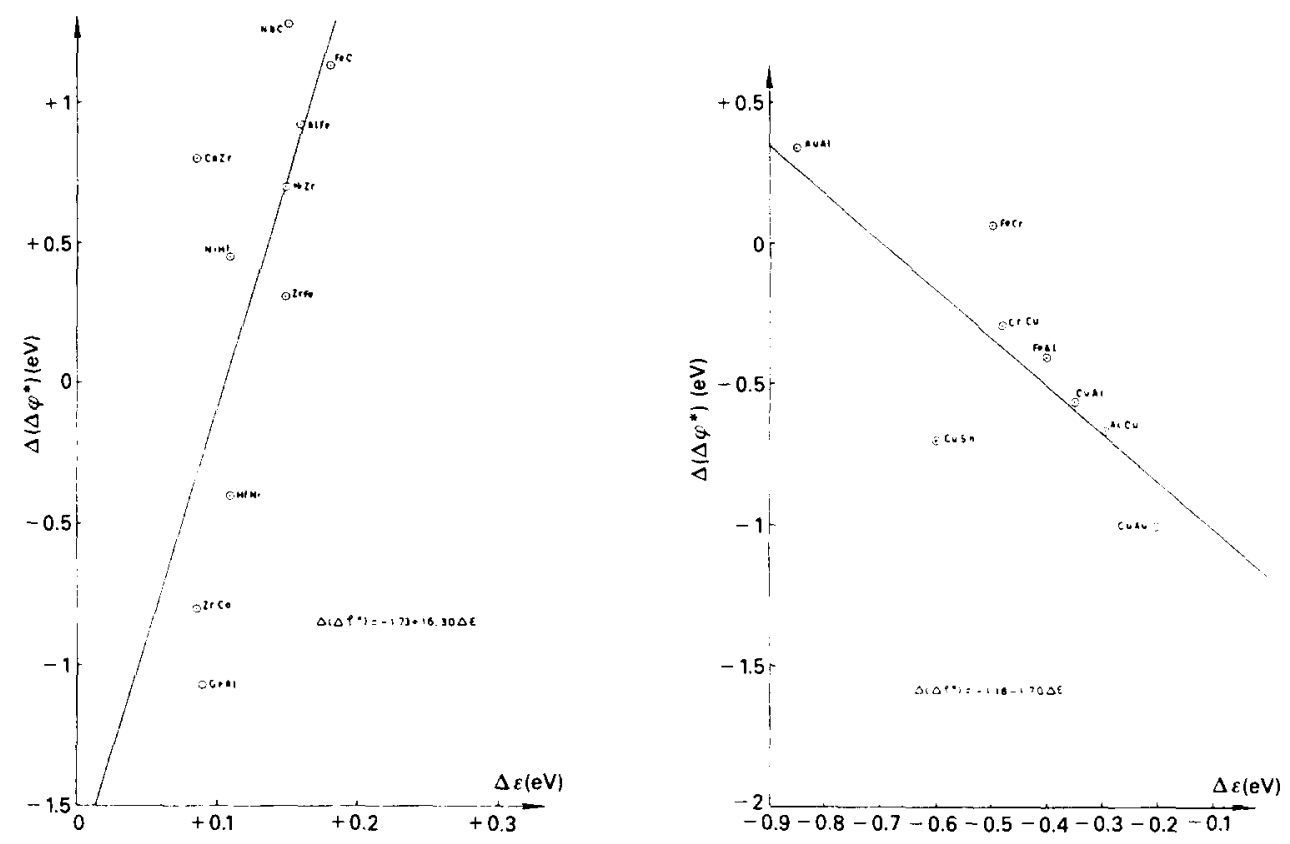

Fig. 66. Calculated $\Delta\left(\Delta \phi^{*}\right)$ vs $\Delta \mathrm{E}$ relation of a) amorphous and b) crystalline alloys. (from ref. 238)

A physical interpretation of the model is as follows. Positive $\Delta \mathrm{E}$ values for amorphous alloys mean that the charge density adjustment is possible only at the expense of a net increment in electronic energy, which contributes to destabilization of the system through freezing of a metastable disordered structure. The reverse holds for negative $\Delta \mathrm{E}$ values for crystalline alloys. Segregation behavior of alloys reflects variation in the surface energy $\Delta\left(\Delta \phi^{*}\right)$ between components of A-B and C-D alloys. A negative $\Delta\left(\Delta \phi^{*}\right)$, i.e., a smaller difference in the surface energy between A-B alloy elements is indicative of enhanced surface atomic mobility. This reflects a decrease in atomic coordination numbers towards a situation typical of the liquid state, with many energetically equivalent local atomic configurations. In such a situation, glass formation is likely to occur via fast quenching of highly uncorrelated atomic motions. Conversely, positive $\Delta\left(\Delta \phi^{*}\right)$ values correspond to a reduced surface atomic mobility. Stronger interatomic correlation favors the attainment of static configurations and thus crystallization. 
Ossi [238] notes that charge transfer mechanisms generally are effective in driving crystal instability towards glass formation. The same type of local ordering effects found in crystals is operative in establishing compositional SRO in liquids and amorphous materials. In turn, compositional SRO is thought to be essential to stabilize disorder produced inside cascades. Compositional SRO alterations are reproduced in the SCT model by surface energy changes, i.e., variations in $\Delta\left(\Delta \phi^{*}\right)$.

Benyagoub and Thome [239,240] formulated a quantitative model for the amorphization of an alloy under ion bombardment. They assume that the crystalline-to-amorphous transition in ion-bombarded metallic alloys generally results from the combination of two effects: disorder production (radiation damage) and stabilization of the disorder by the establishment of a favorable CSRO (already existing in the alloy or brought by implanted ions). Since the amorphous volume in the ion-bombarded system changes continuously with ion fluence, it is clear that the transition is not global but occurs locally. They then assume that the amorphization takes place by the formation of amorphous islands (clusters) as soon as the concentration of defects and stabilizer atoms locally exceed a given threshold concentration. Their model consists of a description of the amorphous fraction at a given depth in a crystal subjected to ion bombardment at a given temperature.

Several alloy systems were studied using both heavy and light ion irradiation over a range of temperatures. Irradiation experiments yield a sigmoidal shape of the amorphization kinetics in implantation experiments or irradiation experiments with very light ions, while a nearly linear fluence dependence of the amorphous fraction is observed in irradiation experiments with heavy ions. Values of the critical ion concentration $c_{c}$ and critical volumes $v_{c}$ of the amorphous clusters formed can be extracted from the fits to experimental data using this statistical representation. It is shown that $c_{c}$ varies very little with the alloy considered and is always small compared to the concentrations required in similar alloys prepared by fast-quenching techniques.

It was also noted that at temperatures where ions and defects are frozen in the host alloy, the values of $v_{c}$ seem to depend on the nature and composition of the alloy rather than on the mass of the irradiating ion. Further, the critical radius of an amorphous cluster formed by ion bombardment grows as the temperature is decreased. Also noted was that the amorphization cross section increases with the irradiating ion mass while $d_{c}$, the defect density, is nearly independent of the mass. At room temperature, the number of ion impacts needed to amorphize a given region of the sample is generally the same as that at low temperature but the amorphization cross section is lower, certainly due to 
a high rate or defect recombination. The model cannot account, however, for the irradiation of crystalline metallic alloys with heavy ions.

\section{Additional microstructural effects of ion implantation}

In addition to the changes in the phase microstructure of the surface during irradiation, changes to the microstructure of the surface such as densification, grain growth, texturing, dislocation formation and recrystallization also occur.

(i) Densification. Densification effects are easiest to observe during ion beam assisted deposition when the film is being applied with the assistance of an ion beam. Since the as-deposited film is often porous, the increase in surface mobility due to the comcomitant bombardment with an ion beam results in significant increases in density. The density of $\mathrm{ZrO}_{2}$ films on borosilicate crown glass using electron beam evaporation, and an ion beam of $600 \mathrm{eV}$ Ar was found to have its highest density at a beam current density of $50 \mu \mathrm{A} / \mathrm{cm}^{2}$ [241]. IBAD of $\mathrm{CeO}_{2}$ at $300^{\circ} \mathrm{C}$ showed a packing density increase from 0.55 to 1.0 [242]. Densification of TiN films by $30 \%$ prepared by reactive IBAD using thermal evaporation of $\mathrm{Ti}$ in a $\mathrm{N}_{2}$ partial pressure of $1.3 \times 10^{-3} \mathrm{~Pa}$, and bombarded with $40 \mathrm{keV} \mathrm{Ti}$ ions and an arrival rate ratio of $\mathrm{Ti}$ ions/ $\mathrm{Ti}$ atoms of 0.014 [243]. It is expected that similar effects occur in metallic systems.

(ii) Ion-induced grain growth. Ion induced grain growth has been observed by several investigators [244-247] during irradiation of pure metals and multilayers. Liu and Mayer [244] observed the growth of grains of pure nickel films upon irradiation with inert gas ions, $\mathrm{Ar}, \mathrm{Kr}$ and $\mathrm{Xe}$ in the energy range 150 to $580 \mathrm{keV}$. In their experiments, the grain size increased with dose until saturation at about $1 \times 10^{16} \mathrm{i} / \mathrm{cm}^{2}$. They found a nearly homogeneous grain size in the irradiated samples as compared to a wide spread in the grain size of thermally annealed samples. They also observed a dependence of the saturated grain size on ion species and only a weak dependence of grain size on ion dose at high doses, Fig. 67. They suggest that the localized damage caused by the displacement spike in the vicinity of the grain boundary is the driving force for grain growth. The observed grain growth is explained by the reordering or growth of heavily damaged grains onto neighboring, undamaged grains. The reduction in energy at a localized growing grain is equivalent to the difference 
between the energy released from the consumed region and the energy required to expand the grain boundary. The initially rapid grain growth can be explained by a larger probability of damaging an entire grain when the grains are small. As the irradiation process continues, the large grains consume the small ones and the average grain size increases. When the average diameter of the growing grains approaches the dimension of the damaged volume, the probability of highly damaging an entire grain by a single collision cascade is reduced, as is the chance of growing certain grains at the expense of others. Therefore, the grain growth rate gradually decreases with the increase of grain size.
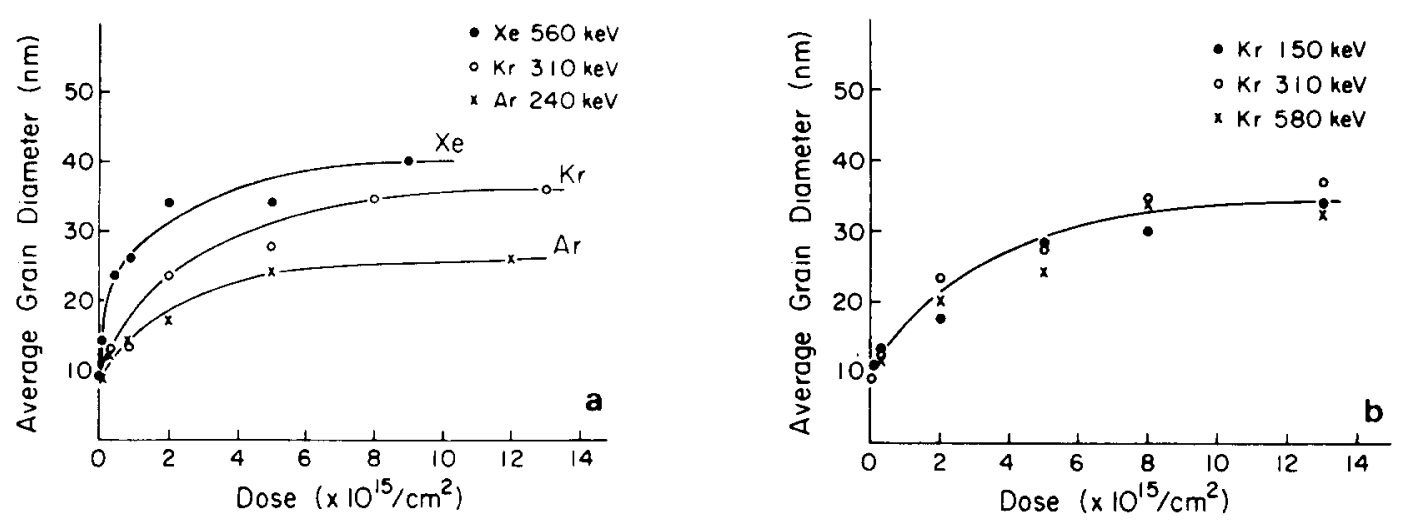

Fig 67. Average grain diameter vs ion dose for a) $240 \mathrm{keV} \mathrm{Ar}, 310 \mathrm{keV} \mathrm{Kr}$ and $560 \mathrm{keV} \mathrm{Xe}$ ion irradiations on polycrystalline $\mathrm{Ni}$ films, and for b) $150 \mathrm{keV}, 310$ $\mathrm{keV}$ and $580 \mathrm{keV} \mathrm{Kr}$ ion irradiation on polycrystalline Ni films. (from ref. 244)

Atwater et al. [245] studied grain growth in thin films of $\mathrm{Ge}$ irradiated with $\mathrm{Ge}, \mathrm{Xe}, \mathrm{Kr}$ and Ar ions. Based on the observed time and temperature dependence of ion beam enhanced grain growth, they proposed that Frenkel defects created at or near grain boundaries were responsible for grain boundary mobility enhancement. They use the linear collision cascade model for defect generation to argue that the number of jumps across the boundary per defect generated is between 1 and 2.5 and that the grain growth rate is proportional to the concentration of point defects, regardless of whether they are generated thermally or by an ion beam.

Recently, Alexander et al. [246] have shown an enhanced grain growth rate during mixing of multilayered $\mathrm{Ni}-\mathrm{Al}$ films as compared with co-deposited $\mathrm{Ni}$-Al 
films of the same average composition. The difference in growth rate was a factor of 2.2, Fig. 68. An attempt to explain the observed differences based on the heat of mixing was made using Johnson's expression for the total number of atom jumps induced in a spike per unit length of a cylindrical thermal spike, $n$. Assuming that this value is proportional to grain boundary mobility as suggested by Liu [244], the grain size can be related to $\mathrm{n}$ as follows:

$$
\left(D^{3}-D_{0}{ }^{3}\right) / \Phi \propto F_{D}^{2} / \Delta H_{\operatorname{coh}^{2}}\left\{1+C\left(\Delta H_{m i x} / \Delta H_{c o h}\right)\right\} .
$$

Since the $\Delta \mathrm{H}_{\mathrm{mix}}=0$ for the irradiated coevaporated films, the ratio of measured values of mobilities should be,

$$
\left(D^{3}-D_{0}^{3}\right) /\left.\Phi\right|_{M L} /\left(D^{3}-D_{0}^{3}\right) /\left.\Phi\right|_{C O}=1+C \Delta H_{m i x} / \Delta H_{c o h} .
$$

Given the cohesive energy and heat of mixing of a $\mathrm{Ni}-20$ at\% $\mathrm{Al}$ alloy, the ratio in eqn (4.12) is 3.0, as compared with the measured value of 2.2. These results indicate that the heat of mixing appears to play a role in ion induced grain growth as well as ion beam mixing.

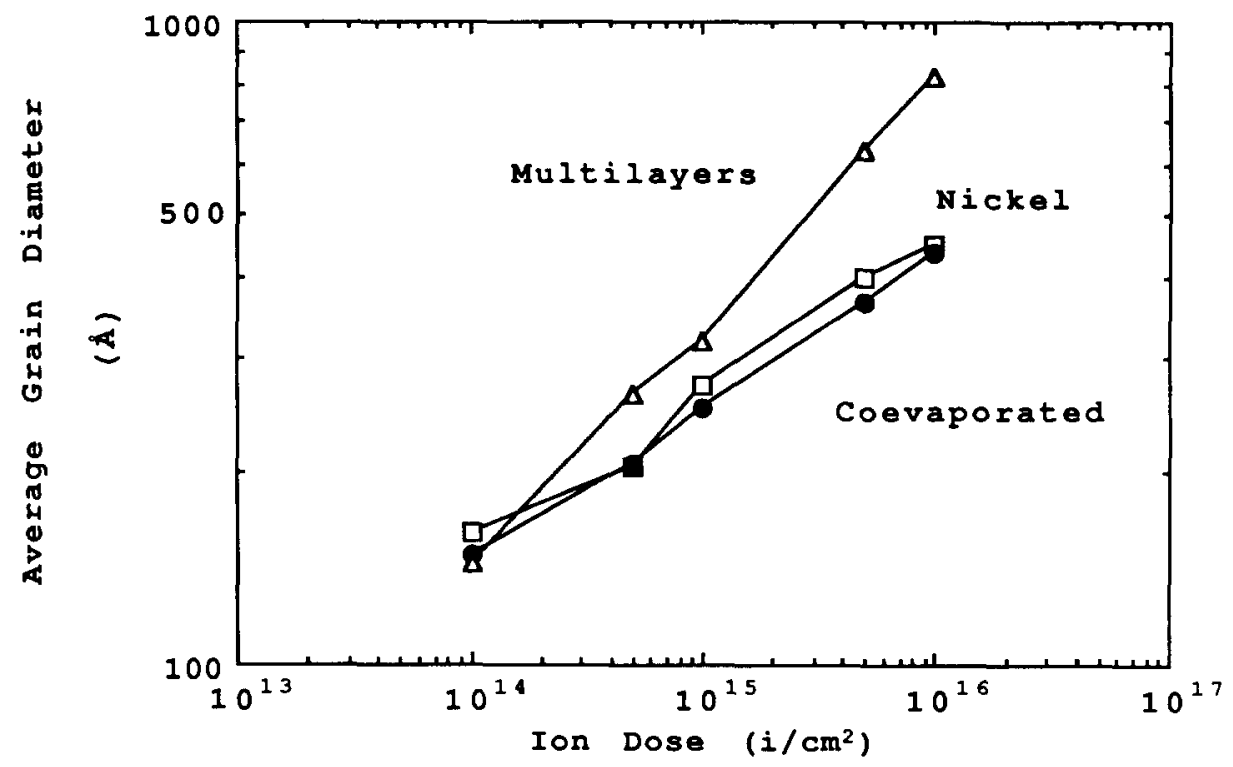

Fig. 68. Ion induced grain growth observed in $40 \mathrm{~nm}$ thick Ni, Ni-20at\% $\mathrm{Al}$ multilayer and $\mathrm{Ni}-20 \mathrm{at} \% \mathrm{Al}$ coevaporated films irradiated with $700 \mathrm{keV} \mathrm{Xe}^{++}$. (from ref. 246) 
Allen and Rehn [247] argue that it is unlikely that thermally activated jumps of point defects generated at boundaries during ion irradiation can account for the phenomenon of irradiation-induced grain growth on the basis of qualitative observations of the response of the microstructure of Au films during heavy ion irradiation [247,248]. In these experiments, the orientation of several grains and subgrains change with time, the regions rocking in and out of contrast as their boundary structures and the orientations of their neighbors change, until coalescence occurs. This dynamic reorientation of subgrains has been recorded in a series of bright field images and shows the highly active concomitant dislocation behavior, which the authors describe as a virtual "anthill" of dislocation activity.

They note that temperatures at which grain growth experiments have been conducted, the vacancies collapse to form faulted partial dislocation loops or stacking fault tetrahedra bounded by partial dislocations. Under the influence of other cascades in the vicinity, the loops and tetrahedra unfault, becoming glissile, and then glide to free surfaces or to grain boundaries. If the structure of a particular grain boundary allows, a dislocation may pass through the boundary or be absorbed and cause emission of a secondary dislocation into the neighboring grain, as has been observed during plastic deformation. Such penetration creates a step in the boundary, that is, an element of boundary migration.

(iii) Texture. There have been many reports of texture effects in ion beam mixed or ion beam assisted deposition of films. Alexander et al. [246] and Eridon et al. [175] found that mixing multilayers of $\mathrm{Ni}$ and $\mathrm{Al}$ in the composition $\mathrm{Ni}_{4} \mathrm{Al}$ resulted in the formation of the hcp and $\mathrm{fcc}(\gamma)$ phases. The $\gamma$ phase had a strong $<111>$ texture and the hcp phase had a $<001\rangle$ texture. The textures were such that the close-packed planes of both phases were parallel to the film surface. These textures formed regardless of the angle of the ion irradiation with respect to the film. The formation of the texture seemed to be driven by the matching of the close packed planes rather than the channeling of the ion beam.

Ahmed and Potter [249] found that irradiation of $\mathrm{Ni}$ with $\mathrm{Al}$ to $1.2 \times 10^{18} / \mathrm{cm}^{2}$ resulted in $3500 \AA$ grains of $\beta^{\prime}$-phase oriented with respect to the underlying fcc nickel in accord with the Nishiyama relationship [250].

The development of texture during ion beam assisted deposition can be more effectively correlated with the channeling directions of ions in the crystal lattice and that the density of energy deposition would be inversely related to the depth of channeling. Thus in an fcc crystal, the ease of channeling is in the order 
$<110\rangle,<200\rangle,<111>$. Bradley et al. [251] have developed a model to explain the development of preferred orientation due to low energy ion bombardment during film growth which is based on the difference in sputtering yields for different orientations rather than reorientation during recrystallization. Both effects are, of course, based on the same phenomena of the variation of energy density with channeling direction. In this model, crystallites with high sputtering orientations are removed more rapidly and newly deposited material grows epitaxially on the low sputtering yield orientations.

Smidt [4] summarizes the observations of ion beam induced texture development as follows: Thin films of PVD deposited materials normally deposit with the planes of highest atomic density parallel to the substrate so fcc films have a $\langle 111\rangle$ texture, bcc films have a $<110\rangle$ texture and hcp films have a $<0002>$ texture (for ideal c/a ratios). The easiest channeling directions in each structure are as follows:

$$
\begin{aligned}
& \text { fcc }:<110\rangle,<100\rangle,<111\rangle \\
& \text { bcc }:<111\rangle,<100\rangle,<110\rangle \\
& \text { hcp }:<11 \overline{2} 0\rangle,<0002>
\end{aligned}
$$

Ion bombardment causes a shift in the preferred orientation to alignment of the easiest channeling direction along the ion beam axis. Thus, an ion beam at normal incidence on an fcc film will cause a shift in orientation from $\langle 111\rangle$ to $<110\rangle$ texture. A beam incident at an angle will produce a different texture depending on the crystallography. The texturing effect appears to be most sensitive to high energy beams because of the larger volume affected per ion and the deeper penetration.

(iv) Microstructural instability: Ion implantation can also induce a high density dislocation network and induce recrystallization which can then affect the distribution of the implanted specie. Ahmed and Potter [249] performed a study of $180 \mathrm{keV} \mathrm{Al}$ implantation into pure $\mathrm{Ni}$ at $25^{\circ} \mathrm{C}$ and at elevated temperatures $\left(300^{\circ} \mathrm{C}-600^{\circ} \mathrm{C}\right)$. At elevated temperatures, individual dislocation loops dominate the microstructure at the lowest fluences $\left(\sim 10^{15} \mathrm{~cm}^{-2}\right)$. These loops bound collections of interstitial atoms or vacancies, defects caused by the energetic Al ions penetrating the nickel structure and displacing atoms from their lattice sites. The loops climb with further implantation, intersect and react with other loops, and form complex dislocation networks after a dose of $2.1 \mathrm{x}$ 
$10^{17} \mathrm{Al} / \mathrm{cm}^{2}$. By a fluence of $3 \times 10^{17} \mathrm{~cm}^{-2}$, three dimensional aggregates of vacancies are present.

The composition profiles at room temperature, 300 and $600^{\circ} \mathrm{C}$ to doses of $6 \mathrm{x}$ $10^{17} \mathrm{~cm}^{-2}$ and $1.2 \times 10^{18} \mathrm{~cm}^{-2}$ are shown in Fig. 69 . Note that there is little difference with temperature. However, at doses in excess of $1.5 \times 10^{18} \mathrm{~cm}^{-2}$, gross changes in the implanted distribution occur with the profile flattening and a considerable amount of Al transported to greater depths, Figs. 70 and 71 . The same occurs following aging of room temperature implantations at $600^{\circ} \mathrm{C}$ for 15 min. The microstructures developed at depths greater than the range of the 180 $\mathrm{keV} \mathrm{Al}^{+}$ions, $\sim 1000 \AA$, play an important role in determining the stability of the implanted concentration profiles.

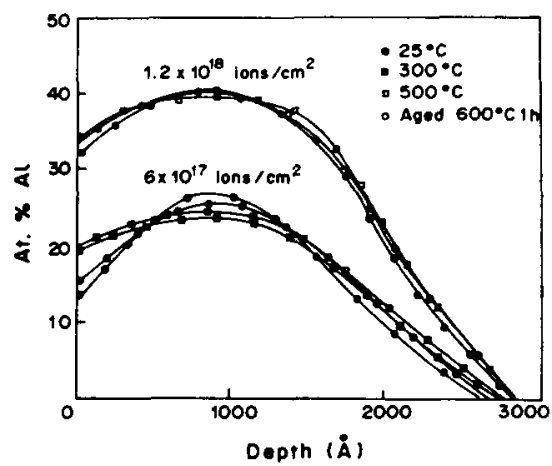

Fig. 69. Composition profiles from specimens implanted to two fluences, as influenced by implantation temperature or aging at $600^{\circ} \mathrm{C}$ after $25^{\circ} \mathrm{C}$ implantation. (from ref. 249)

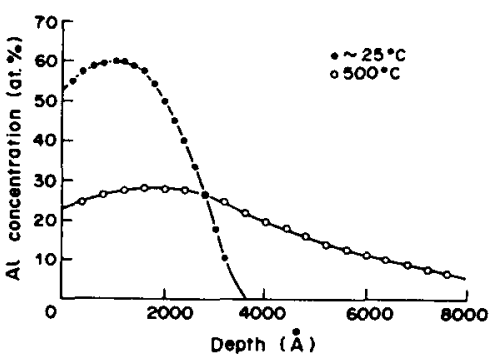

Fig. 70. Aluminum concentration plotted versus depth from a specimen implanted to fluences of $2.4 \times 10^{18} \mathrm{Al}^{+} / \mathrm{c} \mathrm{m}^{2}$ at $25^{\circ} \mathrm{C}$ and $500^{\circ} \mathrm{C}$. (from ref. 249 )

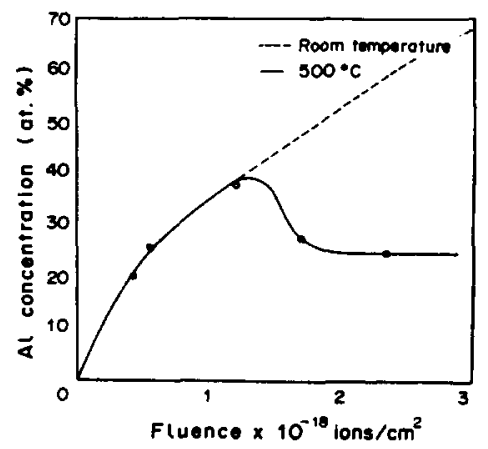

Fig. 71. Peak aluminum concentration plotted versus fluence for implantation at $500^{\circ} \mathrm{C}$. (from ref. 249) 
Dislocation loops are present at depths near $3000 \AA$ following room temperature implantation. These loops are $50-100 \AA$ in diameter and their number density increases with fluence, reaching $\sim 4 \times 10^{16} \mathrm{~cm}^{-2}$ at $3 \times 10^{18} \mathrm{i} / \mathrm{cm}^{-}$ 2. These are determined to be Frank faulted loops. However, the dislocations behind the implanted layer are removed when the material is heated to $600^{\circ} \mathrm{C}$. This occurs by recrystallization which is also responsible for the redistribution of implanted Al. The following describes the processes occurring.

Following room temperature implantation, an amorphous phase extends to a depth of $\sim 1600 \AA$. Small crystal of $\beta^{\prime}$ and $\gamma$ extend from $1600 \AA$ to $\sim 3000 \AA$ and from $\sim 3000 \AA$ to $\sim 4000 \AA$, respectively. Dislocations and dislocation loops extend beyond this to depths of $>8000 \AA$, as shown in Fig. $72 \mathrm{a}$ and b. Aging at $600^{\circ} \mathrm{C}$ following room temperature implantation or implanting at high temperatures causes recrystallization of the fine grain structure to depths of $\sim 8000 \AA$ to $\sim 10,000 \AA$. In both instances, aluminum, atoms must move through relatively pure nickel to accomplish the redistribution which is only possible if some fast diffusion process occurs. This is afforded by the small grains which form upon recrystallization of the heavily dislocated region beyond the implanted layer and provide high angle grain boundaries for abnormally fast diffusion. The composition reaches a plateau by virtue of the limited extent of the recrystallization. Also note, Fig. 73, that the redistribution only occurs above a threshold dose indicating the role of the radiation damage in the recrystallization process. This example serves to tie together the roles of the implanted specie, the character of the radiation damage and the processes (recrystallization and abnormally fast diffusion) that can be affected by implantation.

A final example of the interplay between phase stability, amorphization and irradiation microstructures is the observation of ion induced dendritic growth upon phase transition in Ni-Mo. Huang et al. [252] irradiated multilayered films of $\mathrm{Ni}$ and $\mathrm{Mo}$ with $200 \mathrm{keV} \mathrm{Xe}$ at $-77^{\circ} \mathrm{C}$ and $25^{\circ} \mathrm{C}$. Dendrites were observed in the $\mathrm{Ni}_{65} \mathrm{Mo}_{35}$ and Ni55Mo45 films irradiated to an ion fluence of $7 \times 10^{14} \mathrm{Xe} / \mathrm{cm}^{2}$ at $-77^{\circ} \mathrm{C}$, Fig. 74. Analyses by selected area diffraction indicated that the branches of the dendrite were crystalline while the matrix was partially amorphized. Since a larger ion dose amorphized the entire structure, the film must have been at a critical state of the crystalline-to-amorphous phase transition. The authors argue that since bulk diffusion in a solid film is hard to realize under the given experimental conditions, the diffusing species would then be the atoms, while the nucleus acted as the trap. During growth, other nuclei would be formed and the growth of these nuclei was limited by the existence of others. Thus growth can be characterized as self-limited, due to the superimposition of individual diffusion fields. 


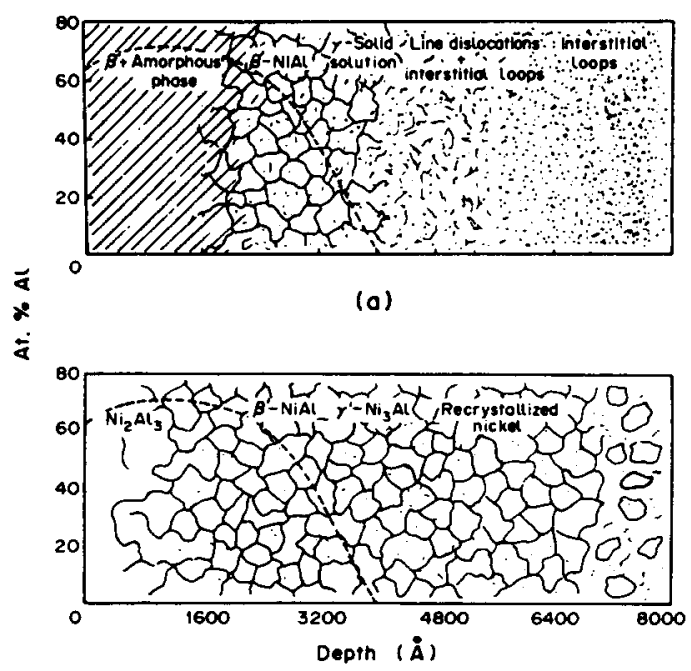

(b)

Fig. 72. Schematic representation of the microstructure observed experimentally as a function of depth: (a) in the room temperature implantation condition, and (b) after a few minutes at $600^{\circ} \mathrm{C}$, for fluences $\geq 1.5 \times 10^{18} \mathrm{i} / \mathrm{cm}^{2}$. (from ref. 249)

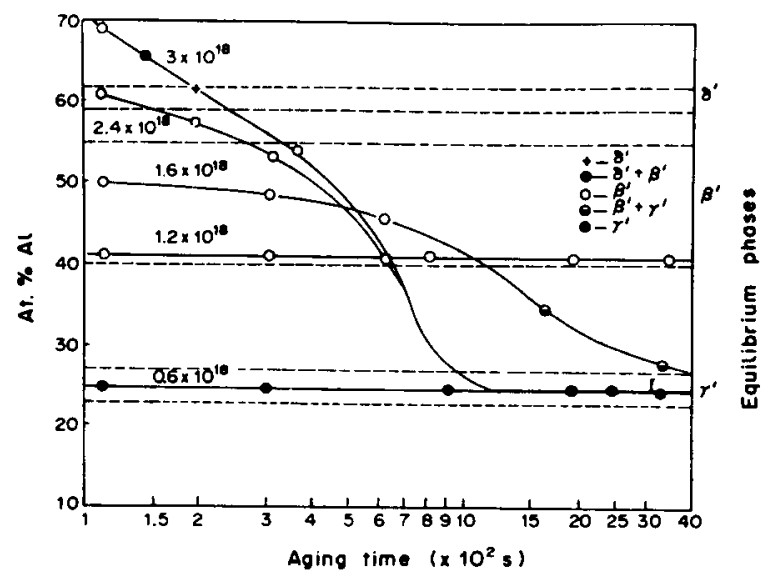

Fig. 73. Aluminum concentration versus time at $600^{\circ} \mathrm{C}$ for fluences (ions $/ \mathrm{cm}^{2}$ ) irradiated. The phases observed in the implanted layers, symbols on curves, and those expected based on the equilibrium diagram, right side ordinate, are both shown. (from ref. 249) 


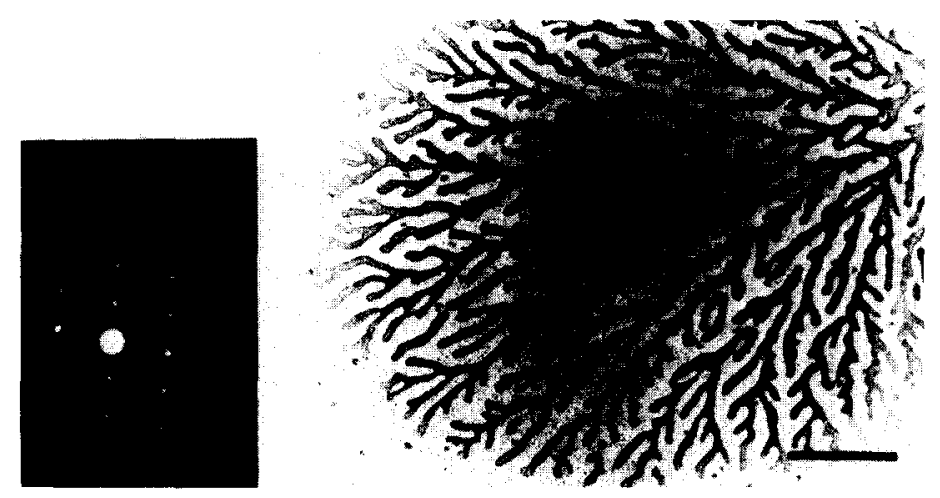

Fig. 74. Observed fractal pattern (Ni55 Mo45) precipitated from an amorphous matrix with a dose of $7 \times 10^{15} \mathrm{Xe}^{+} / \mathrm{cm}^{2}$ (marker bar indicates $2 \mu \mathrm{m}$ ). (from ref. 252)

\section{Summary}

The modification of metals with ion beams can be done in several ways. This paper discussed the most prominent and most commercially promising techniques: direct ion implantation, ion beam mixing, ion beam assisted deposition and plasma source ion implantation. Each has its advantages as well as its drawbacks. However, the principles by which compositional and microstructural tural changes are caused are essentially the same.

The compositional changes induced in a metal under ion bombardment are a result of a number of complex processes occurring simultaneously. on of the prime effects is the relocation of atoms in the solid caused by recoil implantation and cascade mixing. However, the kinetics of the relocation process is not only ballistically driven, but is also a function of the elements involved, giving rise to so-called "chemical effects." The creation of excess point defects and new defects types gives rise to enhanced diffusion termed radiation-enhanced diffusion. The preferential participation of one defect over another in the flux of defects to sinks at elevated temperatures, gives rise to radiation-induced segregation. The measurements of radiation-induced 
segregation will be influenced by the process of Gibbsian adsorption which is the readjustment of the surface composition of a homogeneous alloy in an effort to minimize the free energy of the system. Added to this already extensive list is the process of sputtering or ejection of atoms from the target by the incoming ions. This can also take the form of preferential sputtering where the ejection of elements in a multicomponent alloy are unequal. Taken together, these physical processes present a challenge in determining the composition profile in an alloy under irradiation. Nevertheless, the preceding sections have shown that great strides have been made in understanding these processes individually as well as synergistically. However, this is only half the story - the other being the development in microstructure.

Ion bombardment has been shown to induce the precipitation of second phases. Subsequent irradiation can either cause the precipitates to grow or dissolve, depending on a great many factors. Perhaps of greatest interest is the discovery that ion bombardment can induce the formation of metastable phases. This generally occurs by one of four types of transformation: order <--> disorder, crystal structure A $-->$ crystal structure B, crystal structure A <--> amorphous, and crystal structure A $->$ quasicrystalline. Theories for the formation of metastable phases involve the size of the implanted ion relative to the size of the atoms of the host, the sign of the heat of compound formation, the size and complexity of the unit cell, the solubility range of compounds, the density of defects in the alloy and the characteristics of the alloy which allow accommodation of the point defects into lower energy extended defect structures. Finally, changes to the microstructure directly, such as densification, grain growth, texture and dislocation structure, all occur under ion bombardment. While not present in as integrated a package as the processes governing compositional changes, the preceding sections show that much progress has been made toward understanding of microstructural changes under ion bombardment.

From a practical point standpoint, the interest in understanding compositional and microstructural changes in metals and alloys is for the purpose of altering physical and mechanical properties. Since these properties are a strong function of composition and microstructure, property improvement by ion bombardment techniques will only come through an understanding of the effect on composition and microstructure. Hence, the stage is set for significant progress in improving physical and mechanical properties by ion implantation of metals and alloys. 


\section{References}

1. J. W. Mayer, L. Eriksson and J. A. Davies, Ion Implantation in Semiconductors, Academic Press, New York, 1970.

2. Ion Beam Processing of Advanced Electric Materials, Vols 45 \& 147, Materials Research Society, Pittsburgh, PA 1985 \& 1989.

3. G. Dearnaley, Rep. Prog. Phys., 32, 405 (1969).

4. F. Smidt, International Materials Reviews, in press.

5. J. R. Conrad, J. L. Radtke, R. A. Dodd, F. J. Worzala and N. C. Tran, J. Appl. Phys., 62, No. 11, 4591 (1987).

6. G. H. Kinchin and R. S. Pease, Rep. Progr, Phys, 18, 1 (1955).

7. P. Sigmund, L. Appl. Phys, 50, No. 11, 7261 (1979).

8. P. Sigmund and A. Gras-Marti, Nucl. Instr. Meth., 182/183, 25 (1981).

9. A. Gras-Marti and P. Sigmund, Nucl. Instr, Meth., 180, 211 (1981).

10. J. Lindhard, M. Scharff and H.E. Schiott, Kgl. Danske Videnskab. Selskab Mat.-Fys. Medd., 33, No. 14, 1 (1963).

11. K.B. Winterbon, P. Sigmund, and J.B. Sanders, Kgl. Danske Videnskab. Selskab Mat.-Fys, Medd., 37, No. 14, 1 (1970).

12. Z.L. Liau, J.W. Mayer, W.L. Brown and J. M. Poate, J. Appl. Phys., 49, 5295 (1978).

13. B. M. Paine, L. Appl. Phys., 53, No. 10, 6828 (1982).

14. S. Mantl, L. E. Rehn, R. S. Averback and L. J. Thompson Jr., Nucl. Instr. Meth.s B7/8, 622 (1985).

15. B. W. Auner, Y.-T. Cheng, M. H. Alkisi and K. R. Padmanabhan, Presented at the Materials Research Symposium Fall Meeting, Symposium A, paper A3.5, Boston, November, 1989.

16. M. L. Roush, O. F. Goktepe, T. D. Andreadis and F. Davarya, Nucl, Instr., Meth., 194, 611 (1982).

17. U. Littmark, Nucl. Instr. Meth., B7/8, 684 (1985).

18. H. H. Andersen, Appl. Phys., 18, 131 (1979).

19. S. Matteson and M.-A. Nicolet, Ann. Rev. Mater. Sci., 13, 339 (1983).

20. P. Sigmund and Gras-Marti, Nucl. Instr. Meth, 168, 389 (1980).

21. S. Matteson, J. Roth and M.-A. Nicolet, Radiat. Eff., 42,217 (1979).

22. S. Matteson, B. M. Paine, M. G. Grimaldi, G. Mezey and M.-A. Nicolet, Nucl. Instr. Meth., 182/183, 43 (1981).

23. H. Westendorp, Z. L. Wang and F. W. Saris, Nucl. Instr. Meth., 194, 453 (1982).

24. Z. L. Wang, J. F. M. Sestendorp, S. Doorn and F. W. Saris, in Metastable Materials Formation by Ion Implantation, S. T. Picraux and W. J. Choyke, eds., Elsevier, New York (1982) p. 59.

25. S. T. Picraux, D. M. Follstaedt and J. Delafond, in Metastable Materials Formation by Ion Implantation, S. T. Picraux and W. J. Choyke, eds., Elsevier, New York (1982) p. 71.

26. B. M. Paine and R. S. Averback, Nucl. Instr, Meth., B7/8, 666 (1985).

27. C. Fu-Zhai and L. Heng-De, Nucl. Instr. Meth., B7/8, 650 (1985). 
28. F. d'Heurle, J. E. E. Baglin and G. J. Clark, J. Appl, Phys, 57, No. 4, 1426 (1985).

29. Y.-T. Cheng, M. Van Rossum, M.-A. Nicolet and W. L. Johnson, Appl. Phys. Lett., 45, No. 2, 185 (1984).

30. W. L. Johnson, Y.-T. Cheng, M. Van Rossum and M.-A. Nicolet, Nucl, Instr, Meth., B7/8, 657 (1985).

31. T. W. Workman, Y.-T. Cheng, W. L. Johnson and M.-Al. Nicolet, Appl. Phys. Lett., 50, No. 21, 1485 (1987).

32. Y.-T. Cheng, T. W. Workman, M.-A. Nicolet and W. L. Johnson, in Beam-Solid Interactions and Transient Processes, vol. 74, Materials Research Society, Pittsburgh (1987) p. 419.

33. E. Ma, T. W. Workman, W. L. Johnson and M-A. Nicolet, Appl, Phys. Lett., 54, No. 5, 413 (1989).

34. S. Matteson, B. M. Paine and M.-A. Nicolet, Nucl. Instr. Meth., 182/183, 53 (1981).

35. T. Diaz de la Rubia, R. S. Averback, R. Benedek and W. E. King, Phys, Rev, Lett., 59, No. 17, 1930, (1987).

36. R. P. Webb, D. E. Harrison, Jr. and K. M. Barfoot, Nucl. Instr. Meth., B7/8, 143 (1985).

37. E. Ibe, Nucl. Instr. Meth, B39, 148 (1989).

38. R. Sizmann, J. Nucl, Mater., 69\&70, 386 (978).

39. H. Wiedersich, Radiat. Eff., 12, 111 (1972).

40. S. Matteson, J. Roth and M.-A. Nicolet, Radiat. Eff., 42, 217 (1979).

41. N. Q. Lam, S. J. Rothman and R. Sizmann, Radiat. Eff, 23, 53 (1974).

42. Y.-T. Cheng, X.-A. Zhao, T. Banwell, T.W. Workman, M.-A. Nicolet and W.L. Johnson, J. Appl. Phys, 60, No. 7, 2615 (1986).

43. G. J. Dienes and A. C. Damask, I. Appl, Phys., 29, 1713 (1958).

44. S. M. Myers, Nucl. Instr. Meth, 168, 265 (1980).

45. D. Peak and R. S. Averback, Nucl. Instr, Meth, B7/8, 561 (1985).

46. B. Rauschenbach, Phys, stat. sol. (a), 102, 645 (1987).

47. L. E. Rehn and P. R. Okamoto, Nucl, Instr, Meth, B29, 104 (1989).

48. P. R. Okamoto and H. Wiedersich, J. Nucl. Mater, 53, 336 (1974).

49. R. A. Johnson and N. Q. Lam, Phys. Rev. B, 13, No. 10, 4364 (1976).

50. R. A. Johnson and N. Q. Lam, J. Nucl, Mater, 69\&70, 424 (1978).

51. L. E. Rehn, P. R. Okamoto, D. I. Potter and H. Wiedersich, J. Nucl, Mater, 74, 242 (1978).

52. N. Q. Lam, P. R. Okamoto and R. A. Johnson, L. Nucl, Mater., 78, 408 (1978).

53. A. D. Marwick, J. Phys. F: Metal Phys., 8, No. 9, 1849 (1978).

54. P. R. Okamoto and L. E. Rehn, J. Nucl, Mater, 83, 2 (9179).

55. A. D. Marwick, R. C. Piller and P. M. Sivell, L. Nucl. Mater, 83,35 (1979).

56. H. Wiedersich, P. R. Okamoto and N. Q. Lam, J. Nucl. Mater., 83, 98 (1979).

57. H. Wiedersich, in Physics of Radiation Effects in Crystals, R. A. Johnson and A. N. Orlov, eds., Elsevier Sci. Pub. B.V. (1986) p. 225.

58. H. Wiedersich and N. Q. Lam, in Phase Transformations During Irradiation, F. V. Nolfi, Jr., ed., Applied Science Publishers, LTD, New York (1983) p. 1.

59. R. C. Piller and A. D. Marwick, J. Nucl. Mater, 83, 42 (1979). 
60. L. E. Rehn, P. R. Okamoto, D. I. Potter and H. Wiedersich, in Effects of Radiation on Structural Materials, ASTM STP 683, J. A. Sprague and D. Kramer, eds., American Society for Testing and Materials, Philadelphia (1979) p. 184.

61. A. D. Marwick and R. C. Piller, Radiat. Eff, 47, 195 (1980).

62. L. E. Rehn, in Metastable Materials Formation by Ion Implantation, S. T. Picraux and W. J. Choyke, eds., Elsevier Science Publishing Company, New York (1982) p. 17.

63. R. S. Averback, L. E. Rehn, W. Wagner, H. Wiedersich and P. R. Okamoto, Phys. Rev. B, 28, No. 6, 3100 (1983).

64. L. E. Rehn, P. R. Okamoto and H. Wiedersich, J. Nucl, Mater, 80, 172 (1979).

65. T. Muroga, P. R. Okamoto and H. Wiedersich, Radiat, Eff. Lett, 68 , 163 (1983).

66. L. Kornblit and A. Ignatiev, J. Nucl. Mater, 126, 77 (1984).

67. H.W. King, in Alloying Behavior and Effects in Concentrated Solid Solutions, T. B. Massalski, ed., Gordon and Breach, New York (1965) p. 85.

68. J. E. Hobbs and A. D. Marwick, "Measurements of Radiation-Induced Segregation and Diffusion in an Ion Irradiated Alloy Using Implanted Manganese," AERE-R11528, UKAEA-Harwell, Nov. 1984.

69. J. M. Titchmarsh and I. A. Vatter in Radiation-Induced Sensitisation of Stainless Steels, D. I. R. Norris, ed., Berkeley Nuclear Laboratories, Berkeley, Gloucestershire, GL13 9PB (1987) p. 74.

70. A. D. Marwick and R. C. Piller in Ion Beam Modification of Materials, R. E. Benesen, E. N. Kaufman, G. L. Miller and W. W. Scholz, eds., North-Holland, New York (1981) p. 121.

71. T. R. Anthony in Atomic Transport in Solids and Liquids, A. Lodding and T. Lagerwall, eds., Zeitschrift für Naturforschung, Tübingen (1971) p. 138.

72. L. E. Rehn and H. Wiedersich in Surface Alloying by Ion, Electron and Laser Beams, L. E. Rehn, S. T. Picraux and H. Wiedersich, eds., American Society for Metals, Metals Park, OH (1986) p. 137.

73. K.-H. Robrock in Phase Transformations During Irradiation, F. V. Nolfi, Jr., ed., Appl. Sci. Publishers, LTD, New York (1982) p. 115.

74. H. Wiedersich, P. R. Okamoto and N. Q. Lam in Raidation Effects in Breeder Reactor Structural Materials, M. L. Bleiberg and I. W. Bennett, eds., AIME, New York (1977) p. 801.

75. H. Wiedersich and P. R. Okamoto in Interfacial Segregation, W. C. Johnson and J. M. Blakely, eds., American Society for Metals, Metals Park, OH (1978) p. 405 .

76. P. D. Dederichs, C. Lehmann, H. R. Schober, A. Scholz and R. Zeller, J. Nucl. Mater, 69\&70, 176 (1978).

77. L. E. Rehn, P. R. Okamoto, R. S. Averback, W. Wagner and H. Wiedersich, Scr. Metall., 16, 639 (1982).

78. I. Potter and A. W. McCormick, Acta Metall., 27, 933 (1976).

79. P. Wynblatt and R. C. Ku in Interfacial Segregation, W. C. Johnson and J. M. Blakely, eds., American Society for Metals, Metals Park, OH (1979) p.115.

80. N. Q. Lam and H. Wiedersich, Nucl. Instr. Meth., B18, 471 (1987). 
81. N. Q. Lam, H. A. Hoff, H. Wiedersich and L. E. Rehn, Surf, Sci., 149, 517 (1985).

82. N. Q. Lam, H. A. Hoff and P. G. Régnier, L. Vac. Sci. Technol, A3, 2152 (1985).

83. M. J. Kelley, P. W. Gilmore and D. G. Swartzfäger, L. Vac. Sci, Technol., 17, 634 (1980).

84. P. Sigmund, Phys, Rev, 184, 383 (1969).

85. P. Sigmund, Phys, Rev, 187, 768 (1969).

86. P. Sigmund, in Sputtering by Particle Bombardment I, Springer-Verlag, New York (1981) p. 9.

87. H. F. Winters, in Radiation Effects in Solid Surfaces, M. Kaminsky, ed., American Chemical Society, Washington (1976) p. 1.

88. J. Lindhard, V. Nielsen, M. Scharff and P. V. Thomsen, Kgl. Danske Videnskab. Selskab. Mat,-Fys. Medd., 33, No. 10, 1119119 (1963).

89. J. Lindhard, V. Nielsen and M. Scharff, Kgl. Danske Videnskab. Selskab. Mat.-Fys. Medd., 36, No. 10,1 (1968).

90. E. S. Mashkova and V. A. Molchanov, Radiat. Eff, 108, 307 (1989).

91. Y. Yamamura, N. Matsunami and N. Itoh, Radiat. Eff, 7, 65 (1983).

92. A. Oliva, R. Kelly and G. Falcone, Nucl. Instr. Meth., B19/20, 101 (1987).

93. R. Kelly, Nucl. Inst. Meth., B18, 338 (1987).

94. G. Falcone, Radiat. Eff. Lett., $\underline{85}, 75$ (1984).

95. G. Falcone, R. Kelly and A. Oliva, Nucl. Instr. Meth., B18, 399 (1987).

96. G. N. Van Wyk and A. H. Lategan, Radiat. Eff. Lett., 68, 107 (1982).

97. R. Kelly and D. Harrison, Mater. Sci. Engin, 69, 449 (1985).

98. H. Wiedersich, H. H. Andersen, N. Q. Lam, L. E. Rehn and H. W. Pickering, in Surface Modification and Alloying, J. M. Poate, G. Foti and D. C. Jacobson, eds., NATO Series on Materials Science, Plenum Press, New York (1983) p. 261.

99. P. Sigmund, Nucl, Instr, Meth, B27, 1 (1987).

100. G. Falcone and P. Sigmund, Appl. Phys., 25, 307 (1981).

101. N. Q. Lam, G. K. Leaf and H. Wiedersich, J. Nucl. Mater., 88, 289 (1980).

102. N. Q. Lam and H. Wiedersich, in Metastable Materials Formation by Ion Implantation, S. T. Picraux and J. W. Choyke, eds., North-Holland, New York (1982) p. 35.

103. L. E. Rehn, N. Q. Lam and H. Wiedersich, Nucl. Instr. Meth., B7/8, 764 (1985).

104. R. Shimizu, Nucl. Instr. Meth., B18, 486 (1987).

105. H. Shimizu, M. Ono and K. Nakayama, Surf. Sci, , 36, 817 (1973).

106. K. Goto, T. Koshikawa, K. Ichimura and R. Shimizu, in Proc. 7 th Int. Vac. Cong. and 3rd Int. Conf. on Solid Surfaces, Vienna (1977) p. 1493.

107. R. Shimizu and T. Okutani, Proc. 4th Symp. on Ion Sources and Applications Technology/1980, Ionics., Tokyo (1980) p. 273.

108. M. Shikata and R. Shimizu, Surf. Sci., 97, L363 (1980).

109. T. Okutani, M. Shikata, R. Shimizu, Surf. Sci., 99, L410 (1980).

110. O. G. Swartzfäger, S. B. Ziefecki and M. J. Kelly, J. Vac. Sci. Technol., 19, 185 (1981). 
111. Ri-Sheng Li, T. Koshikawa ans K. Goto, Surf, Sci, 121, L561 (1982).

112. T. Koshikawa, Appl. Surf. Sci, 22/23, 118 (1985).

113. H.J. Kang, R. Shimizu and T. Okutani, Surf, Sci, 115, L175 (1982).

114. N. Q. Lam and H. Wiedersich, J. Nucl, Mater., 103/104, 433 (1981).

115. N. Q. Lam and G. K. Leaf, J. Mater, Res., 1, 251 (1986).

116. L. E. Rehn and P. R. Okamoto, in Phase Transformations During Irradiation, F. V. Nolfi, Jr., ed., Applied Science Publishers, New York (1983) p. 247.

117. S. G. B. Mayer, F. F. Milillo and D. I. Potter, in High-Temperature Ordered Intermetallic Alloys, C. C. Koch, C. T. Liu and N. S. Stoloff, eds., vol. 39 Materials Research Society, Pittsburgh (1985) p. 521.

118. H. Wiedersich, Rad. Eff, 101, 21 (1986).

119. K. C. Russell, in Prog in Materials Science, J. Christian, P. Haansen and T. B. Massalski, eds., 28, No. 3/4, Pergamon Press, Oxford (1984) p. 229.

120. G. Martin, R. Cauvin and A. Barbu, in Phase Transformations During Irradiation, F. V. Nolfi, ed., Applied Science Publishers, New York (1983) p. 46.

121. H. J. Frost and K. C. Russell, in Phase Transformations During Irradiation, F. V. Nolfi, ed., Applied Science Publishers, New York (1983) p. 75.

122. A. J. Ardell and K. Janghorban, in Phase Transformations During Irradiation, F. V. Nolfi, ed., Applied Science Publishers, New York (1983) p. 291.

123. J. L. Katz and H. Wiedersich, J. Colloid. Interface, 2, 351 (1977).

124. M. Mruzik and K. C. Russell, J. Nucl. Mater, 78, 343 (1978).

125. S. I. Maydet and K. C. Russell, J. Nucl. Mater, 64, 101 (1977).

126. R. Cauvin and G. Martin, Phys. Rev. B23, 3322 (1981).

127. H. Wiedersich, P. R. Okamoto and N. Q. Lam, L. Nucl. Mater, 83, 98 (1979).

128. N. Q. Lam, P. R. Okamoto, H. Wiedersich and A. Taylor, Metall. Trans., 9A, 1767 (1978).

129. K.-H. Robrock and P. R. Okamoto, in Comportement Sous Irradiation des Materiaux Metalliques et des Composants des Coeuis des Reacteurs Rapides, J. Poirier and J. M. Dupuoy, eds., Commissariat a l'Energie Atomique, Gif-sur-Yvette, (1979) pp. 57-60.

130. H. R. Brager and F. A. Garner, J. Nucl. Mater., 73, 9 (1978).

131. H. R. Grager and F. A. Garner, in Effects of Radiation on Structural Materials, J. A. Sprague and D. Kramer, eds., American Society for Testing and Materials, ASTM STP 633, Philadelphia (1979) p. 207.

132. H. R. Brager and F. A. Garner, J. Nucl. Mater., 116, 267 (1983).

133. U. K. Sethi and P. R. Okamoto, in Phase Stability During Irradiation, J. R. Holland, L. K. Mansur and D. I. Potter, eds., The Metallurgical Society of AIME, Warrendale (1981) p. 109.

134. T. M. Williams, R. M. Boothby and J. M. Titchmarsh, in Radiation Induced Sensitization of Stainless Steel, D. I. R. Norris, ed., Berkeley Nuclear Labs., Berkeley, Gloucestershire, GL13 9PB (1987) P. 116.

135. E. H. Lee, A. F. Rowcliffe and E. A. Kenik, L. Nucl. Mater. 83, 79 (1979).

136. R. A. Nelson, J. A. Hudson and D. J. Mazey, J. Nucl, Mater, 44, 318 (1972).

137. K. C. Russell, J. Nucl. Mater., 83, 176 (1979).

138. C. F. Bilsby, I. Nucl. Mater. 55, 125 (1975). 
139. M. Baron, A. Chang and M. L. Bleiberg in Radiation Effects in Breeder Reactor Structural Materials, M. L. Bleiberg and J. W. Bennett, eds., The Metallurgical Society of AIME, Warrendale (1977) p. 395.

140, A. D. Brailsford, L. Nucl. Mater, 91, 221 (1980).

141. P. Wilkes, L Nucl. Mater, 83,166 (1979).

142. R. Cauvin and G. Martin, L. Nucl. Mater., 83, 67 (1979).

143. I. M. Lifschitz and V. V. Slyozov, L. Phys. Chem. Solids, 19, 35 (1961).

144. C. Wagner, Z. Electrochem., 65, 581 (1961).

145. D. I. Potter and H. A. Hoff, Acta Metall, 24, 1155 (1976).

146. D. I. Potter and D. G. Ryding, L. Nucl. Mater., Z1, 14 (1977).

147. D. I. Potter and H. Wiedersich, J. Nucl. Mater, 83, 208 (1979).

148. D. I. Potter, P. R. Okamoto, H. Wiedersich, J. R. Wallace and A. W. McCormick, Acta Metall., 27, 1175 (1979).

149. K. Urban and G. Martin, Acta Metall, 30, 1209 (1982).

150. A. Vom Felde, J. Fink, Th. Müller-Heinzering, J. Pflüger, B. Schurer, G. Linker and D. Koletta, Phys. Rev. Lett., 53, 922 (1984).

151. C. Templier, C. Jaouen, J-P. Riviere, J. Delafond and J. Grilhe, Compter Randus, 299, 613 (1984).

152. A. Luukainen, J. Keionen and M. Erola, Phys. Rev. B32, 4814 (1985).

153. W. Jäger, R. Manzke, H. Trinkous, R. Zeller, J. Fink and G. Crecelius, Rad. Eff., 78,315 (1983).

154. R. C. Birtcher and W. Jäger, Ultramicroscopy, 22, 267 (1978).

155. R. C. Birtcher and W. Jäger, Nucl. Instr. Meth., B15, 435 (1986).

156. J. H. Evans, Nucl. Instr. Meth., B18, 16 (1986).

157. G. W. Greenwood, A. J. E. Foreman and D. E. Rimmer, J. Nucl. Mater., 4, 305 (1959).

158. J. H. Evans and D. J. Mazey, J. Nucl. Mater., 138, 176 (1986).

159. E. M. Schulson, L. Nucl. Mater., 83, 239 (1979).

160. K. Y. Liou and P. Wilkes, J. Nucl. Mater., 87, 317 (1979).

161. R. H. Zee and P. Wilkes, in Phase Stability During Irradiation, The Metallurgical Society of AIME, Warrendale (1981) p. 295.

162. S. Banerjee and K. Urban, Phys. Stat. Sol, (a), 81, 145 (1984).

163. D. I. Potter, L. E. Rehn, P. R. Okamoto and H. Wiedersich, Scr. Metall, 11 , 1095 (1977).

164. S. M. Murphy, Phil. Mag. A, $\underline{58}$, No. 2, 417 (1988).

165. J. R. Manning, Metall. Trans., 1,499 (1971).

166. R. P. Gupta, Phys. Rev.. B22, 5900 (1980).

167. S. M. Murphy, in Proc. BNES Conf. on Materials for Nuclear Reactor Core Applications, Brighton, 1 (1987) p. 301.

168. J. L. Brimhall, H. E. Kissinger and L. A. Charlot, Rad. Eff., 77, 273 (1983).

169. J. L. Brimhall and E. P. Simonen, Nucl. Instr. Meth., B16, 187 (1986).

170. L. S. Hung, and J. W. Mayer, Nucl. Instr. Meth, B7/8, 676 (1985).

171. G. S. Was and J. M. Eridon, Nucl. Instr. Meth, B24/25, 557 (1987).

172. H. Wiedersich, in Radiation Damage in Metals, N. L. Peterson and S. D. Harkness, eds., American Society for Metals, Metals Park, OH (1976) p. 157.

173. A. D. Brailsford and R. Bullough, J. Nucl. Mater, 69\&70, 434 (1978). 
174. D. I. Potter, in Phase Transformations During Irradiation, F. V. Nolfi, Jr., ed., Applied Science Publishers, New York (1983) p. 213.

175. J. Eridon, L. Rehn and G. Was, Nucl. Instr. Meth, B 19/20, 626 (1987).

176. D. E. Luzzi and M. Meshii, Scr, Metall, 20, 943 (1986).

177. J. Koike, P. R. Okamoto, L. E. Rehn, P. Bhadra, M. H. Grimsditch and M. Meshii, Mat. Res. Soc. Symp., vol. (1989), paper A11.3.

178. E. Johnson, T. Wohlenberg and W. A. Grant, Phase Transitions, 1, 23 (1979).

179. W. A. Grant, Nucl. Instr. Meth., 182/183, 809 (1981).

180. E. Johnson, U. Littmark, A. Johnson and C. Christodoulides, Phil. Mag. A, 45, 803 (1982).

181. D. M. Follstaedt, Nucl. Instr. Meth., B7/8, 11 (1985).

182. P. Ziemann, Mater. Sci. Engin, 69, 95 (1985).

183. J. Eridon, G. S. Was and L. Rehn, J. Mater. Res., 3, No. 4, 626 (1988).

184. L. S. Hung, M. Nastasi, J. Gyulai and J. W. Mayer, Appl. Phys. Lett., 42, 622 (1983).

185. M. Nastasi, L. S. Hung and J. W. Mayer, Appl. Phys. Lett., 43, No. 9, 831 (1983).

186. D. A. Lilienfield, L. S. Hung and J. W. Mayer, Nucl. Instr. Meth., B19/20, 1 (1987).

187. D. A. Lilienfield and J. W. Mayer, Mat. Res. Soc. Symp. vol 74, Pittsburgh (1987) p. 339.

188. B. X. Liu, Phys. Stat. Sol. (a), 94, 11 (1986).

189. H. Jones in The Theory of Brillouin Zones and Electronic States in Crystals, North-Holland, Amsterdam (1960) p. 191.

190. B. X. Liu, Phys. Stat. Sol. (a), 75, K77 (1983).

191. B. Y. Tsaur, S. S. Lau and J. W. Mayer, Appl. Phys. Lett., 36, 823 (1980).

192. B. Y. Tsaur and J. W. Mayer, Appl. Phys. Lett., 37, 389 (1980).

193. B. Y. Tsaur, S. S. Lau, L. S. Hung and J. W. Mayer, Nucl. Instr. Meth., 182/183, 67 (1981).

194. J. W. Mayer, B. Y. Tsaur, S. S. Lau and L. S. Jung, Nucl. Instr. Meth., $182 / 183,1$ (1981).

195. B. X. Liu, L. J. Huang, J. Li and E. Ma, in Thin Films - Interfaces and Phenomena, R. J. Nemanich, P. S. Ho and S. S. Lau, eds., Mat. Res. Soc. Symp. Proc., vol. 54, North-Holland, Amsterdam (1986) p. 217.

196. B. X. Liu, E. MA, J. Li and L. J. Huang, Nucl. Instr. Meth., B19/20, 682 (1987).

197. J. Meissner, K. Kopitzki, G. Mertler and E. Peiner, Nucl. Instr. Meth., B19/20, 669 (1987).

198. E. Peiner and K. Kopitzki, Nucl. Instr. Meth, B34, 173 (1988).

199. D. Shectman, I. Blech, D. Gratias and J. W. Chan, Phys. Rev. Lett, 53, 1951 (1984).

200. P. J, Steinhardt, Amer, Sci., 74, 586 (1986).

201. J. A. Knapp and D. M. Follstaedt, Phys. Rev. Lett, 55, No. 15, 1591 (1985).

202. D. A. Lilienfield, M. Nastasi, H. H. Johnson, A. G. Ast and J. W. Mayer, Phys. Rev, Lett., 55, No. 15, 1587 (1985).

203. P. M. Ossi, Mater. Sci. Engin, 90, 55 (1977).

204. D. E. Luzzi, H. Mori, H. Fujita and M. Meshii, Scr. Metall, 18, 957 (1984). 
205. D. F. Pedraza, Rad. Eff, 112, 11 (1990).

206. D. F. Pedraza, Mater. Sci. Engin, 90,69 (1987).

207. D. F. Pedraza and L. K. Mansur, Nucl. Instr. Meth., B16, 203 (1986).

208. D. F. Pedraza, L. Mater. Res. 1, 425 (1986).

209. E. P. Simonen, Nucl. Instr. Meth., B16, 198 (1986).

210. G. Hägg, Z. Phys. Chem, B, 12, 3 (1931).

211. B. Rauschenbach and K. Hohmuth, Phys. Stat. Sol. (a), 72, 667 (1982).

212. W. A. Grant, A. Ali and P. J. Grundy, Nucl, Instr, Meth. 182/183, (1981).

213. J. Hafner, Phys. Rev. B21, 406 (1980).

214 R. Miedema, R. Boom and F. R. DeBoer, J. Less Comm. Metals, 41, 283 (1975).

215. W. W. Hu, C. R. Clayton, H. Hermann, J. K. Hirvonen and R. A. Kant, Rad. Eff., $49,71(1980)$.

216. K. Hohmuth, B. Rauschenbach, A. Kolitsch and E. Richter, Nucl. Instr. Meth., 209/210, 249 (1983).

217. R. Andrew, W. A. Grant, P. J. Grundy, J. S. Williams, and L. T. Chadderton, Nature, 262, 380 (1976).

218. S. R. Nagel and J. Tauc, Phys. Rev. Lett., 35, 380 (1975).

219. B. Rauschenbach and V. Heera, Defect and Diffusion Data, 57-58, 143 (1988).

220. H. M. Naguib and R. Kelly, Rad. Eff, 25, 1 (1975).

221. J. A. Alonso and S. Simozar, Solid State Comm., 48, 765 (1983).

222. A. R. Miedema, L. Less Comm. Met. 32, 117 (1973).

223. B. X. Liu, W. L. Johnson, M-A. Nicolet and S. S. Lau, Nucl. Instr. Mater. 209/210, 97 (1983).

224. B. X. Liu, Wl. L. Johnson, M-A. Nicolet and S. S. Lau, Appl. Phys. Lett., 42 , No. 1, 45 (1983).

225. G. Martin, Phy. Rev, B30, 1424 (1984).

226. M. L. Swanson, J. R. Parsons and C. W. Hoelke, Rad. Eff., 2, 249 (1971).

227. J. L. Brimhall, E. P. Simonen and H. E. Kissinger, J. Nucl. Mater, 48, 339 (1973).

228. J. L. Brimhall and E. P. Simonen, Nucl. Instr. Meth., B16, 187 (1986).

229. J. L. Brimhall, H. E. Kissinger and A. R. Pelton, Rad, Eff., 90, 241 (1985).

230. E. P. Simonen, Nucl. Instr, Meth., B16, 198 (1986).

231. V. Goerlach, P. Ziemann and W. Buckel, Nucl. Instr. Meth., 209/210, 235 (1983).

232. W. Folberth, H. Leitz and J. Hasse, Z. Phys, B43, 235 (1981).

233. A. Schmid and P. Ziemann, Nucl. Instr. Meth., B7/8, 581 (1985).

234. P. R. Okamoto, L. E. Rehn, J. Pearson, R. Bhadra and M. Grimsditch, in Mat. Res. Soc. Symp. Proc. vol. 100, MRS, Pittsburgh (1980) p. 51.

235. W. L. Johnson, Prog. Mater. Sci, 30, 287 (1986).

236. R. W. Cahn and W. L. Johnson, J. Mater, Res., 1, 724 (1986).

237. W. J. Meng, P. R. Okamoto, B. J. Kestel, L. J. Thompson and L. E. Rehn, Appl. Phys. Lett., 53, 7 (1988).

238. P. M. Ossi, Mater. Sci. Engin., Al15, 107 (1989).

239. L. Thome, A. Benyagoub, F. Pons, E. Ligeon, J. Fontenille and R. Danielou, Mater. Sci, Engin., A115, 127 (1989). 
240. A. Benyagoub and L. Thome, Phys. Rev, B, 38, No. 15, 10205 (1988).

241 P. J. Martin, R. P. Netterfield and W. G. Sainty, J. Appl. Phys., 55, 235 (1984).

242. R. P. Netterfield, W. G. Sainty, P. J. Martion and S. H. Sie, Appl. Opt., 24, 2267 (1985).

243. F. A. Smidt, Proc. DOE Workshop on Coatings for Advanced Heat Engines, USDOE, 1988.

244. J. C. Liu and J. W. Mayer, Nucl. Instr. Meth., B19/20, 538 (1987).

245. H. A. Atwater, C. V. Thompson and H. I. Smith, Mat. Res. Soc. Symp. Proc., vol. 100, MRS, Pittsburgh (1987) p. 345.

246. D. E. Alexander, G. S. Was and L. E. Rehn, Mat. Res. Soc. Symp. Proc 1989, paper A6.1.

247. C. W. Allen and L. E. Rehn, Mat. Res. Soc. Symp. Proc., Fall 1989, paper A2.1.

248. J. C. Liu, J. W. Mayer, C. W. Allen and L. E. Rehn, in Processing and Characterization of Materials Using Ion Beams, L. E Rehn, J. Greene and F. A. Smidt, eds., Materials Research Society, Pittsburgh, AP, 1988, Vol. 128, p. 297.

249. M. Ahmed and D. I. Potter, Acta Metall., 35, No. 9, 2341 (1987).

250. Z. Nishiyama, in Martensitic Transformations, M. E. Fine, M. Meshii and C. M. Wayman, eds., vol. 7, Academic Press, New York (1978).

251. R. M. Bradley, J. M. E. Harper and D. A. Smith, J. Appl. Phys., 60, 4160 (1986).

252. L. J. Huang, J. R. Ding, H-D. Li and B. X. Liu, Nucl. Instr. Meth., B39, 144 (1989). 